\title{
CS24-43
}

Screw Threads and Tap-Drill Sizes

U. S. DEPARTMENT OF COMMERCE

JESSE H. JONES, Secretary

NATIONAL BUREAU OF STANDARDS

LYMAN J. BRIGGS, Director

\section{SCREW THREADS AND TAP-DRILL}

\section{SIZES}

\section{COMMERCIAL STANDARD CS24-43}

(Revision and consolidation of CS24-30 and CS25-30)

Effective Date for New Production from February 10, 1943

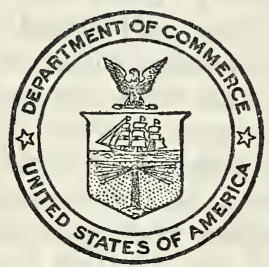

\section{A RECORDED VOLUNTARY STANDARD OF THE TRADE}

UNITED STATES

GOVERNMENT PRINTING OFFICE

WASHINGTON : 1943 


\section{PROMULGATION}

of

COMMERCIAL STANDARD CS24-43

for

\section{SCREW THREADS AND TAP-DRILL SIZES}

(Revision and consolidation of CS24-30 and CS25-30)

At the request of the National Screw Thread Commission, American National screw-thread tables for shop use were circulated January 23, 1930 , as recommended commercial standards to producers, distributors, and users for a written acceptance. They were subsequently accepted in writing by the industry and published under the titles, American National Standard Screw Threads, Coarse and Fine-Thread Series, Commercial Standard CS24-30; and American National Special Screw Threads, Commercial Standard CS25-30.

On July 28,1942 , on the recommendation of the Interdepartmental Screw Thread Committee, and with the endorsement of the standing committee, a consolidation and revision of CS24-30 and CS25-30, under the title of Recommended Commercial Standard for Screw Threads and Tap-Drill Sizes, was circulated for acceptance. Those concerned have since accepted and approved the standard as shown herein for promulgation by the United States Department of Commerce, through the National Bureau of Standards.

The standard is effective for new production from February 10, 1943.

Promulgation recommended.

I. J. Fairchild, Chief, Division of Trade Standards.

Promulgated.

Lyman J. Briggs,

Promulgation approved.

Jesse $\mathrm{H}$. Jones, Secretary of Commerce. 
SCREM THREADS IND TAP-DRILL SIZES

COMMERCIAL STANDARD CS24-43

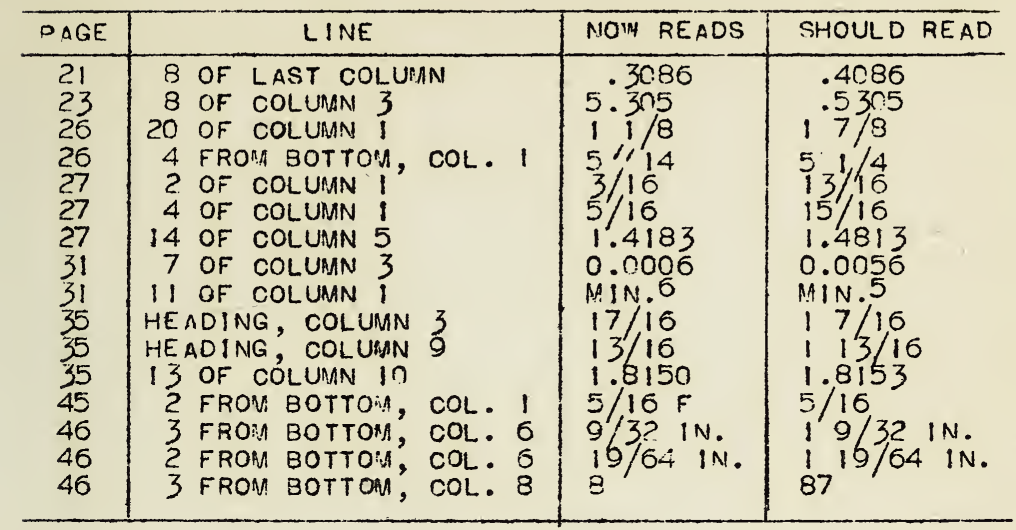





\section{SCREW THREADS AND TAP-DRILL SIZES}

(Revision and Consolidation of CS24-30 and CS25-30)

\section{COMMERCIAL STANDARD CS24-43}

\section{CONTENTS}

Promulgation

Purpose

Scope

Definitions

Symbols

Specifications

American National coarse-thread series

American National fine-thread series

Uniform-pitch screw-thread series for high-pressure fastenings, boiler applications, machinery components, etc

Form of thread

Thread series

American National 12-pitch-thread series

American National 16-pitch-thread series..-.-.-.

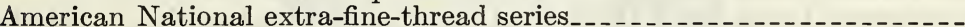

Form of thread.....

Thread series

Labeling.

Effective date

History of project

Acceptance of commercial standard

To the acceptors

Acceptors.

Index

Commercial standards

\section{PURPOSE}

1. The purpose of this standard is to make available for convenient shop use and acceptance inspection the essential specifications, definitions, and dimensional data on screw threads and tap drills, which are recorded more completely in "Screw Thread Standards for Federal Services, 1942," National Bureau of Standards Handbook $\mathrm{H} 28$.

\section{SCOPE}

2. This standard covers the predominating sizes of American National Screw Threads in the following series and fits, with the corresponding tap-drill sizes:

Coarse-thread series, sizes No. $1\left(0.073^{\prime \prime}\right)$ to $4^{\prime \prime}$, classes $1,2,3$, and 4 fits.

Fine-thread series, sizes No. $0\left(0.060^{\prime \prime}\right)$ to $1 \frac{1}{2}{ }^{\prime \prime}$, classes $1,2,3$, and 4 fits. 
8 -pitch-thread series, sizes $1^{\prime \prime}$ to $6^{\prime \prime}$, classes 2 and 3 fits. 12-pitch-thread series, sizes $1 / 2$ " to $6^{\prime \prime}$, classes 2 and 3 fits. 16-pitch-thread series, sizes $3 / 4^{\prime \prime}$ to $4^{\prime \prime}$, classes 2 and 3 fits. Extra-fine-thread series, sizes $1 / 4$ " to $2^{\prime \prime}$, classes 2 and 3 fits. Tap drills for No. 1 to $33 / 4$ "' coarse-thread series.

Tap drills for No. 0 to $1 \frac{1 / 2}{2}$ fine-thread series.

Tap drills for $1^{\prime \prime}$ to $3 \frac{1}{2}{ }^{\prime \prime} 8$-pitch-thread series.

Tap drills for $1 / 2$ " to $3 \frac{1 / 2}{2}$ 12-pitch-thread series.

Tap drills for $3 / 4^{\prime \prime}$ to $3 \frac{1 / 2}{\prime \prime}$ 16-pitch-thread series.

Tap drills for $1 / 4$ " to 2 " extra-fine-thread series.

\section{DEFINITIONS}

3. Terms relating to screw threads and illustrations of terminology.

3a. Numbering of tables and figures. - Since most of the figures and tables herein are identical with those in National Bureau of Standards Handbook H28, they are numbered identically for convenient cross reference, even though this results in some numerical discontinuity in this standard. Figures 1, 2, 3, and 10 illustrate the terms and symbols as defined.

3b. Screw thread.-A ridge of uniform section in the form of a helix on the external or internal surface of a cylinder, or in the form of a conical spiral on the external or internal surface of a cone.

3c. External and internal threads. ${ }^{1}$ - An external thread is a thread on the outside of a member. Example: A threaded plug.

An internal thread is a thread on the inside of a member. Example: A threaded hole.

3d. Major diameter.-The largest diameter of the thread of the screw or nut. The term "major diameter" replaces the term "outside diameter" as applied to the thread of a screw and also the term "full diameter" as applied to the thread of a nut.

3e. Minor diameter. - The smallest diameter of the thread of the screw or nut. The term "minor diameter" replaces the term "core diameter" as applied to the thread of a screw and also the term "inside diameter" as applied to the thread of a nut.

3f. Pitch diameter.-On a straight screw thread, the diameter of an imaginary cylinder, the surface of which would pass through the threads at such points as to make equal the width of the threads and the width of the spaces cut by the surface of the cylinder. On a taper screw thread, the diameter, at a given distance from a reference plane perpendicular to the axis of an imaginary cone, the surface of which would pass through the threads at such points as to make equal the width of the threads and the width of the spaces cut by the surface of the cone.

3g. Pitch.- The distance from a point on a screw thread to a corresponding point on the next thread measured parallel to the axis.

$$
\text { The pitch, in inches, }=\frac{1}{\text { Number of threads per inch }} \cdot
$$

3h. Lead.-The distance a screw thread advances axially in one turn. On a single-thread screw the lead and pitch are identical; on

1 These terms are here defined because of possible confusion arising from the fact that an "internal member" has an "external thread," and vice versa. For the sake of brevity, an external thread is hereinafter referìed to as a "screw," and an internal thread as a "nut." 
a double-thread screw the lead is twice the pitch; on a triple-thread screw the lead is three times the pitch, etc.

3i. Angle of thread.- The angle included between the sides of the thread measured in an axial plane.

3j. Half angle of thread.-The angle included between a side of the thread and the normal to the axis, measured in an axial plane.

3k. Helix angle.-The angle made by the helix, or conical spiral, of the thread at the pitch diameter with a plane perpendicular to the axis.

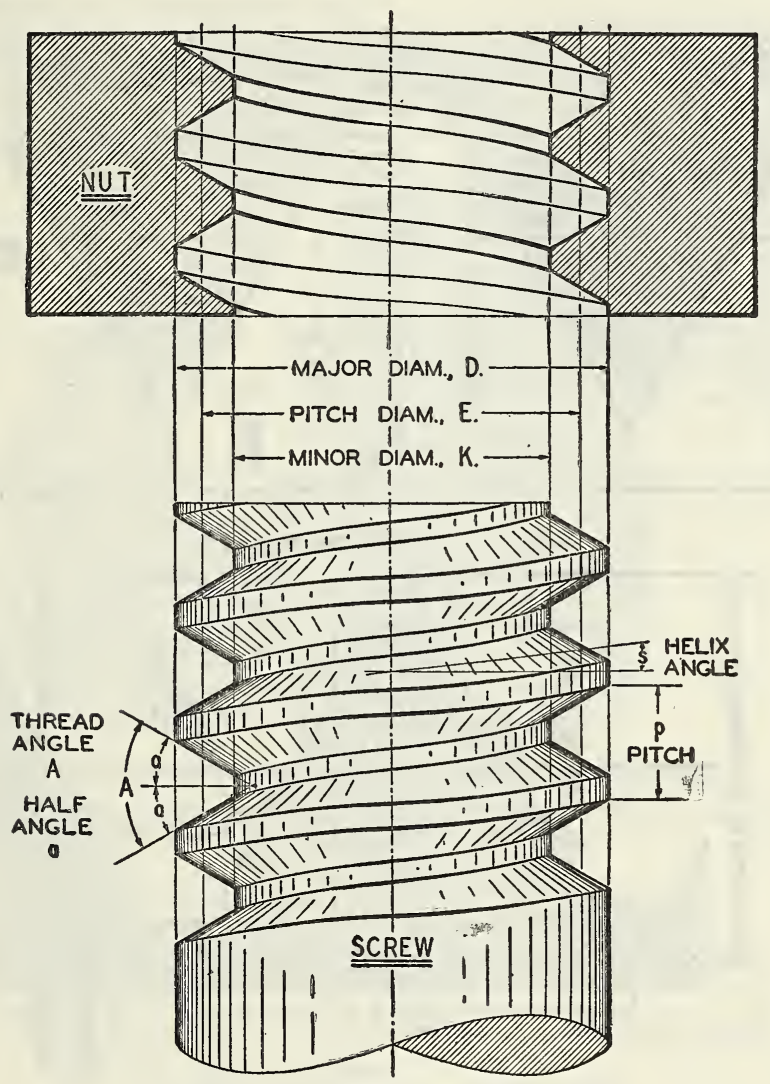

FIGURE 1.-Screw-thread notation.

31. Crest.- The surface of the thread corresponding to the major diameter of the screw and the minor diameter of the nut.

$3 \mathrm{~m}$. Root.-The surface of the thread corresponding to the minor diameter of the screw and the major diameter of the nut.

3n. Side or flank.- The surface of the thread which connects the crest with the root.

3o. Axis of a screw.-The longitudinal central line through the screw.

3p. Base of thread.-The bottom section of the thread; the greatest section between the two adjacent roots. 
3q. Depth of thread.-The distance between the crest and the base of the thread measured normal to the axis.

3r. Number of threads. - Number of threads in 1 inch of length.

3s. Length of engagement.-The length of contact between two mated parts, measured axially.

3t. Depth of engagement.- The depth of thread contact of two mated parts, measured radially.

3u. Pitch line.-An element of the imaginary cylinder or cone specified in definition $3 \mathrm{f}$.

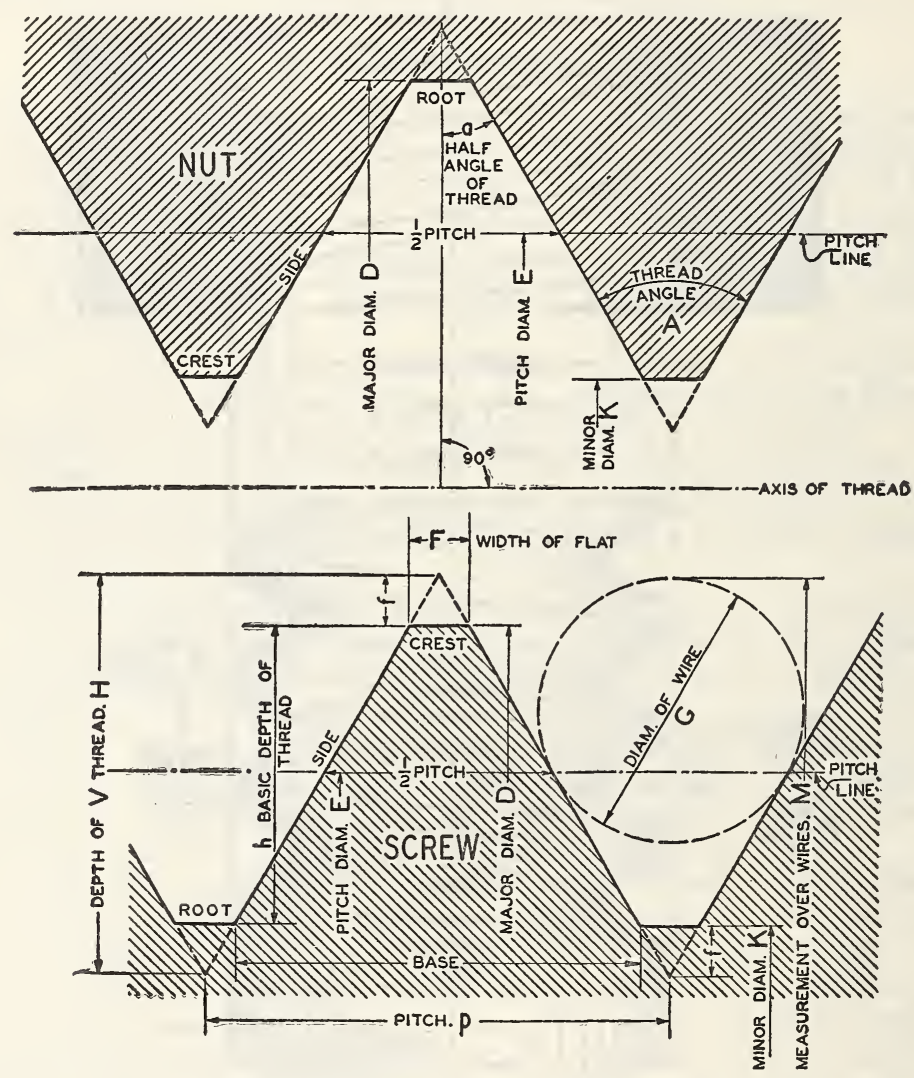

FIGURE. 2-Screw-thread notation.

$3 \mathrm{v}$. Thickness of thread.-The distance between the adjacent sides of the thread measured along or parallel to the pitch line.

3w. Mean area.-The term "mean area of a screw", when used in specifications and for other purposes, designates the cross-sectional area computed from the mean of the basic pitch and minor diameters.

4. Terms relating to classes of fit and tolerances.

4a. Allowance.-An intentional difference in the dimensions of mating parts. It is the minimum clearance or the maximum interference which is intended between mating parts. It represents the 
condition of the tightest permissible fit, or the largest internal member mated with the smallest external member.

Example:

One-half inch, class 1 fit, American National coarse-thread series:

Minimum pitch diameter of nut

Maximum pitch diameter of screw

Allowance (positive)

0.0022

One-half inch, class 4 fit, American National coarse thread series:

Minimum pitch diameter of nut__._._. 0.4500

Maximum pitch diameter of screw

Allowance (negative)

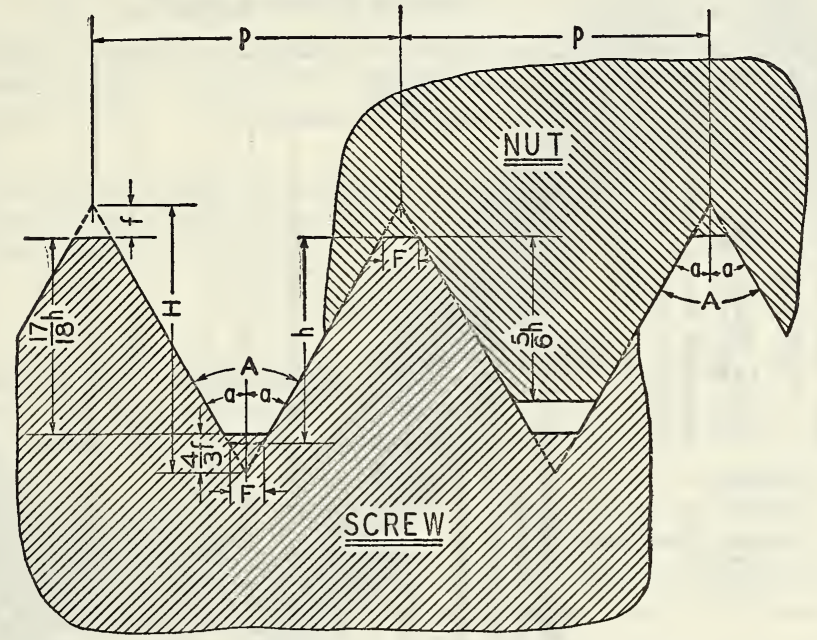

FigURE 3.-American National form of thread.

Note.- No allowance is shown. This condition exists in classes 2 and 3 fits, where both the minimum nut and the maximum screw are basic.

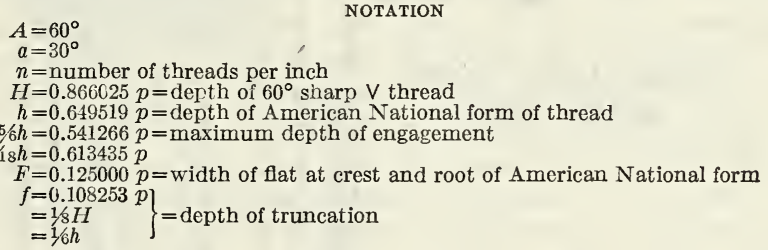

4b. Tolerance.-The amount of variation permitted in the size of a part. Example:

One-half-inch screw, class 1 fit, American National coarse-thread series:

Maximum pitch diameter. 4478

Minimum pitch diameter

Tolerance

0. 0074

4c. Basic size.-The theoretical, or nominal, standard size from which all variations are made. 
4d. Crest clearance.-Defined on a screw form as the space between the crest of a thread and the root of its mating thread.

4e. Finish.-The character of the surface on a screw thread or other product.

4f. Fit.-The relation between two mating parts with reference to the conditions of assembly, for example, classes 1, 2, 3, and 4. Each fit has its proper place, and none should be regarded as superior or

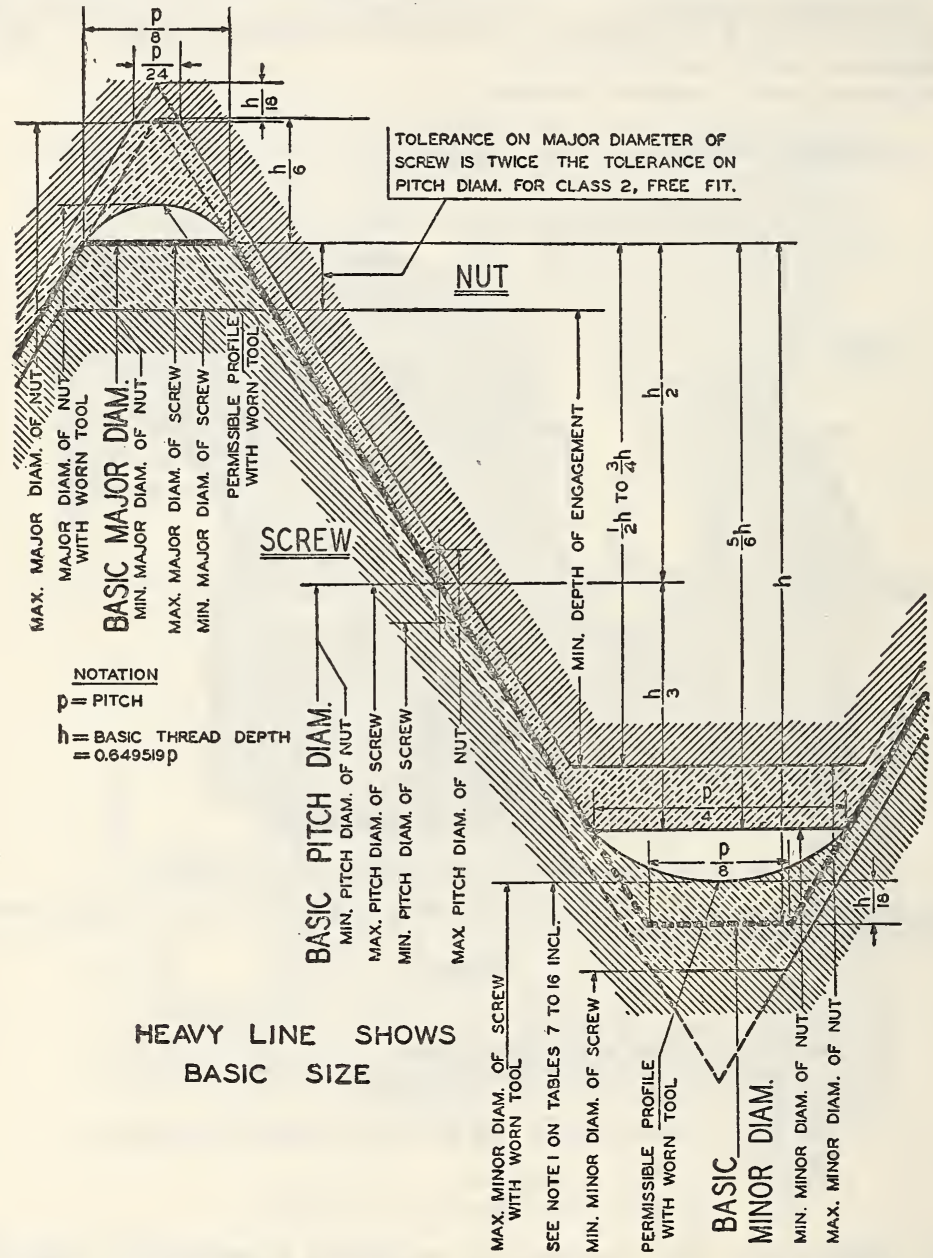

FIGURE 10.-Illustration of tolerances and crest clearances for class 3 fit.

inferior, provided that there is compliance with specification requirements under which it is manufactured and sold.

Class 1 fit includes screw-thread work in which the threads must assemble easily, and where an allowance is required to permit ready assembly, even when the threads are slightly bruised or dirty.

Class 2 fit represents a high quality of commercial screw-thread product and is recommended for the major portion of interchangeable screw-thread work, finished and semifinished bolts and nuts, machine screws, etc., where no allowance is required. 
Class 3 fit is the same in every particular as class 2 fit except that the tolerances are smaller. The class 3 fit is intended to apply to interchangeable screw-thread work requiring the smallest practicable tolerances. Tapped holes within class 3 tolerances are difficult and expensive to produce commercially.

Class 4 fit is designed for screw-thread work where extremely close tolerances are required. In the manufacture of screw-thread products to this class of fit, it will be necessary to use precision tools, gages made to special tolerances, and other refinements. This class of fit should, therefore, be used only in cases where the requirements of the mechanism being produced are exacting, or where there are special conditions which make this class of fit necessary. In order to ensure assembly with the degree of tightness desired, it may be necessary, in some cases, to select the parts when the product is being assembled.

4g. Neutral zone.-A positive allowance. (See Allowance, par. 4a.) 4h. Limits.-The extreme permissible dimensions of a part. Example:

One-half-inch screw, class 1 fit, American National coarse-thread series:

Maximum pitch diameter._._. 0.4478 These are

Minimum pitch diameter

$.4404\}$ the limits.

\section{SYIMBOLS}

5. Symbols for designating screw-thread standards and thread dimensions are a necessity in commercial and engineering practice. The standardization of such symbols yields the usual advantages of standardization. Those listed below have been in customary use for many years, and their general use in standards, specifications, and textbooks is recommended.

6. Identification symbols.-These are for use on correspondence, drawings, shop and storeroom cards, specifications for parts, taps, dies, gages, etc., and on tools and gages.

$6 \mathrm{a}$. The method of designating a screw thread by means of symbols is by the use of the initial letters of the thread series preceded by the diameter in inches (or the screw number) and number of threads per inch, all in Arabic characters, and followed by the classification of fit in Arabic numerals. If the thread is left hand, the symbol "LH" shall follow the class of fit. No symbol is used to distinguish right-hand threads. The number of threads per inch shall be indicated in all cases, irrespective of whether it is the standard number of threads for that particular size of threaded part or special. For screw threads of American National form but of special diameters, pitches, and lengths of engagement, the symbol "NS" shall be used. Examples:

American National coarse-thread series:

To specify a threaded part 1 inch in diameter, 8 threads per inch, class 1 fit

Threaded part 1 inch in diameter, 8 threads per inch, class 2 fit, left hand

Mark

$1^{\prime \prime}-8 \mathrm{NC}-1$

$1^{\prime \prime}-8 \mathrm{NC}-2 \mathrm{LH}$

American National fine-thread series:

Threaded part 1 inch in diameter, 14 threads per inch, class 4 fit

Threaded part $5 / 8$ inch in diameter, 18 threads per inch, class 5 fit.

Threaded part, $1 / 8$ inch in diameter, 44 threads per inch, class 2 fit...........

$700485^{\circ}-46-2$

$$
\begin{aligned}
& 1^{\prime \prime}-14 \mathrm{NF}-4 \\
& 5 / 8^{\prime \prime}-18 \mathrm{NF}-5 \\
& 5-44 \mathrm{NF}-2
\end{aligned}
$$


American National 8-, 12-, or 16-pitch-thread series:

Threaded part 1 inch in diameter, 12 threads per inch, class 3 fit.

Threaded part $11 \%$ inches in diameter, 8 threads per inch, class 2 fit, left hand

American National extra-fine-thread series:

Threaded part 1 inch in diameter, 20 threads per inch, class 3 fit

American National form, special pitch:

Threaded part 1 inch in diameter, 18 threads per inch, class 2 fit.

Threaded part $1 \frac{1}{4}$ inches in diameter, 20 threads per inch, class $3 \mathrm{fit}$, left hand......... \\ $1^{\prime \prime}-12 \mathrm{~N}-3$ \\ $11^{\prime \prime}-8 \mathrm{~N}-2 \mathrm{LH}$ \\ $1^{\prime \prime}-20 \mathrm{NEF}-3$ \\ $1^{\prime \prime}-18 \mathrm{NS}-2$

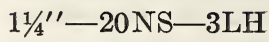

\section{SPECIFICATIONS}

7. American National form of thread.-The form of thread profile specified herein, known previously as the "United States Standard or Sellers' profile", is adopted and shall hereafter be known as the "American National form of thread".

7a. Angle of thread.- The basic angle of thread ( $A$, fig. 3 ) between the sides of the thread measured in an axial plane is $60^{\circ}$. The line bisecting this $60^{\circ}$ angle is perpendicular to the axis of the screw thread.

7b. Flat at crest and root.- The flat at the root and crest of the basic thread form is $1 / 8 \times p$, or $0.125 \times p$.

7c. Depth of thread. - The depth of the basic thread form is

$$
h=0.649519 \times p, \text { or } h=\frac{0.649519}{n},
$$

where

$p=$ pitch in inches.

$n=$ number of threads per inch.

$h=$ basic depth of thread.

7d. Clearance at minor diameter.-A clearance shall be provided at the minor diameter of the nut by removing from the crest of the basic thread form an amount such as to provide a depth of thread not less than 53 to 75 percent (depending on the size), and not more than $83 \frac{1 / 3}{3}$ percent of the basic thread depth.

7e. Clearance at major diameter. - A clearance shall be provided at the major diameter of the nut by making the thread form such that the width of flat shall be less than $1 / 8 \times p$, but not less than $1 / 24 \times p$.

7f. Thread series.-The present coarse-thread and fine-thread series are maintained, the coarse-thread series being the "United States standard" threads, supplemented in the sizes below one-fourth inch by sizes taken from the standard established by the American Society of Mechanical Engineers (ASME). The fine-thread series is composed of standards that have been found necessary and consists of sizes taken from the standards of the Society of Automotive Engineers (SAE) and the fine-thread series of the ASME.

7g. There are indicated in figure 3 the relations as specified herein for the American National form of thread for the minimum nut and maximum screw, classes 2 and 3 fits.

${ }_{2}^{2}$ This standard, in substantially the same form, has been adopted by the American Standards Association. It is published, in part, as ASA Bl. 1-1935, Screw Threads, by the ASME, 29 West 39th Street, New York, N. Y. 


\section{AMERICAN NATIONAL COARSE-THREAD SERIES}

8a. The American National coarse-thread series, as specified in table 1 , is recommended for general use in engineering work, in machine construction where conditions are favorable to the use of bolts, screws, and other threaded components where quick and easy assembly of the parts is desired, and for all work where conditions do not require the use of fine-pitch threads. Limiting dimensions and tolerances for classes 1,$2 ; 3$, and 4 fits are specified in table 15 . 


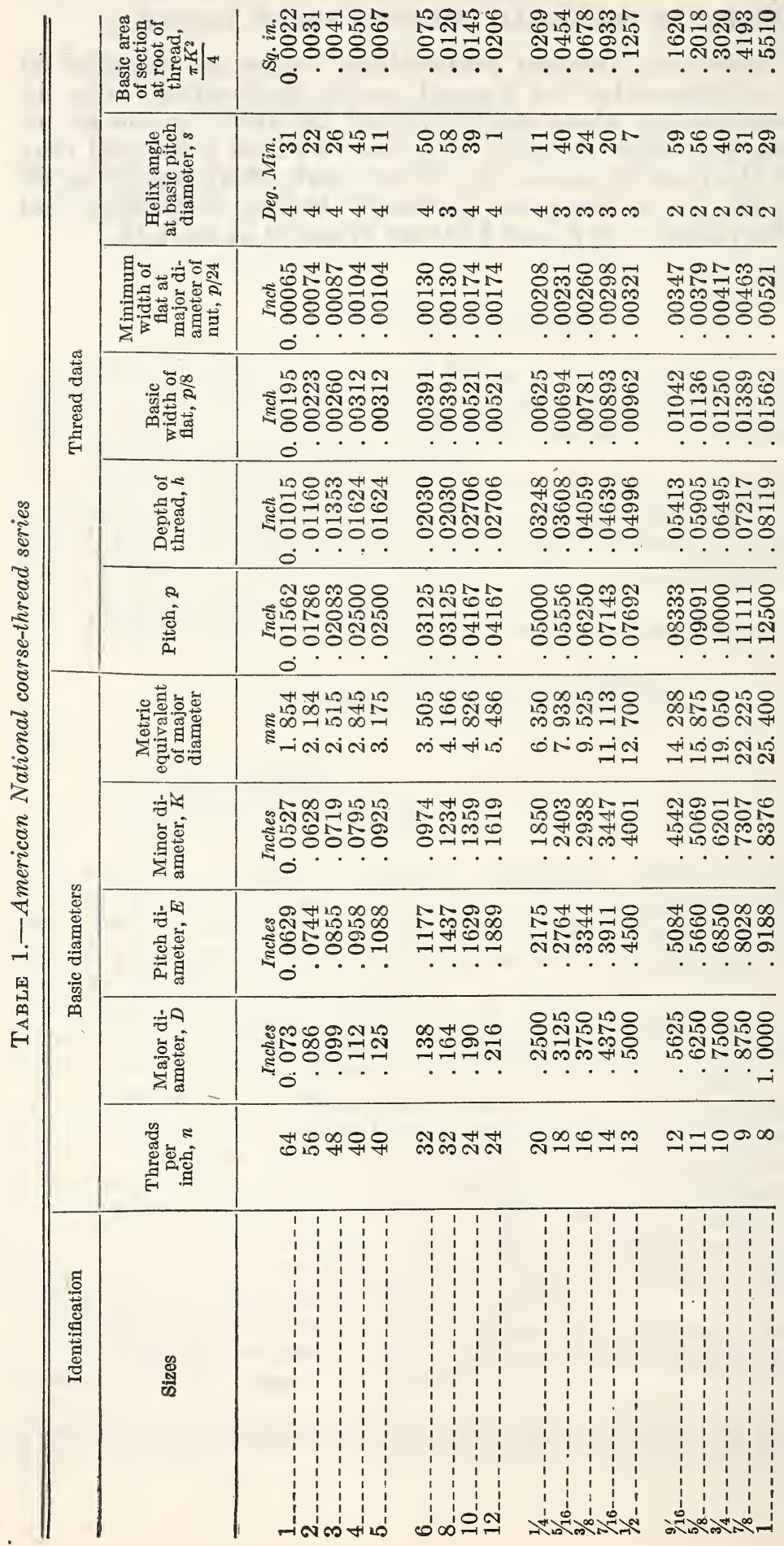




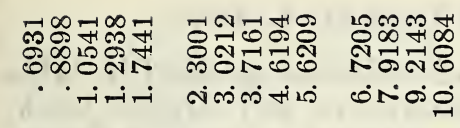

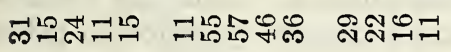

NลNNล

\begin{tabular}{|c|c|c|}
\hline 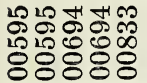 & 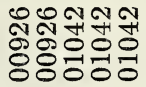 & 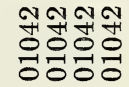 \\
\hline
\end{tabular}

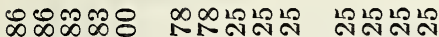
NNO

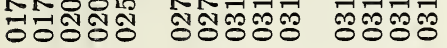

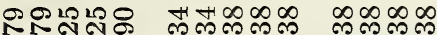
N $\infty \infty 8$ H

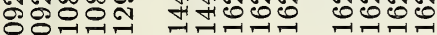

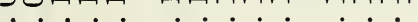

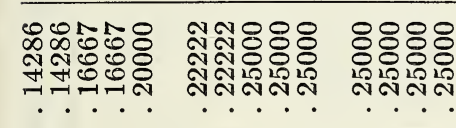

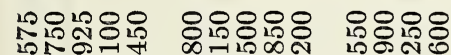

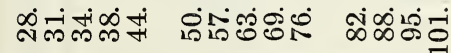

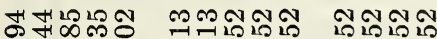

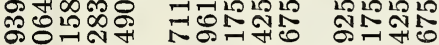

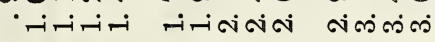

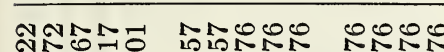
RTST

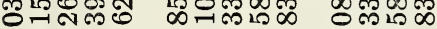

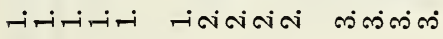

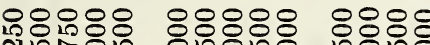
สมต

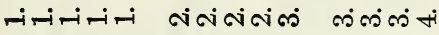

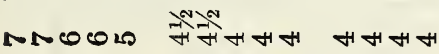

\begin{tabular}{|c|c|c|c|c|c|c|c|c|c|c|c|c|c|}
\hline I & 1 & I & I & I & 1 & I & 1 & 1 & 1 & 1 & 1 & 1 & I \\
\hline I & I & I & 1 & I & I & I & i & i & i & i & $i$ & i & $i$ \\
\hline I & I & I & I & I & 1 & I & i & i & I & i & i & i & i \\
\hline i & i & i & i & $i$ & $i$ & $i$ & $i$ & $i$ & i & i & $i$ & i & $i$ \\
\hline i & i & i & i & i & i & 1 & I & I & 1 & I & 1 & 1 & 1 \\
\hline 1 & I & 1 & I & I & I & I & I & I & i & I & i & I & i \\
\hline i & i & i & i & $i$ & i & i & i & i & i & i & $\mathrm{i}$ & i & $i$ \\
\hline i & I & I & i & I & 1 & I & i & i & i & $i$ & i & i & i \\
\hline i & i & I & i & i & I & i & $i$ & i & $i$ & i & $i$ & $i$ & i \\
\hline I & I & I & 1 & I & I & I & i & I & 1 & I & I & I & i \\
\hline I & I & I & I & I & I & 1 & I & i & i & I & i & i & i \\
\hline i & i & i & $i$ & $i$ & i & i & i & $i$ & i & i & $i$ & i & $i$ \\
\hline I & I & 1 & I & $i$ & I & $i$ & i & i & I & i & $i$ & i & i \\
\hline i & i & I & i & i & i & i & i & i & i & i & $i$ & $i$ & i \\
\hline I & I & 1 & I & I & 1 & 1 & 1 & I & I & 1 & 1 & 1 & I \\
\hline i & i & i & I & I & I & I & i & I & I & i & i & i & i \\
\hline I & I & 1 & I & 1 & I & I & I & I & I & I & I & i & I \\
\hline 1 & I & I & I & I & I & I & i & 1 & I & I & i & I & $i$ \\
\hline 1 & 1 & 1 & 1 & 1 & 1 & 1 & 1 & 1 & 1 & I & 1 & i & I \\
\hline 1 & 1 & I & 1 & 1 & 1 & 1 & I & I & 1 & I & I & I & I \\
\hline 1 & 1 & I & I & 1 & 1 & i & i & i & i & i & i & i & i \\
\hline I & I & i & I & I & & i & i & i & & & i & i & \\
\hline & & & & & & 1 & 1 & & I & 1 & & 1 & i \\
\hline
\end{tabular}


AMERICAN NATIONAL FINE-THREAD SERIES

8b. The American National fine-thread series as specified in table 2 is recommended for general use in automotive and aircraft work, and where special conditions require a fine thread. Limiting dimensions and tolerances for classes 1, 2, 3, and 4 fits are specified in table 16. 


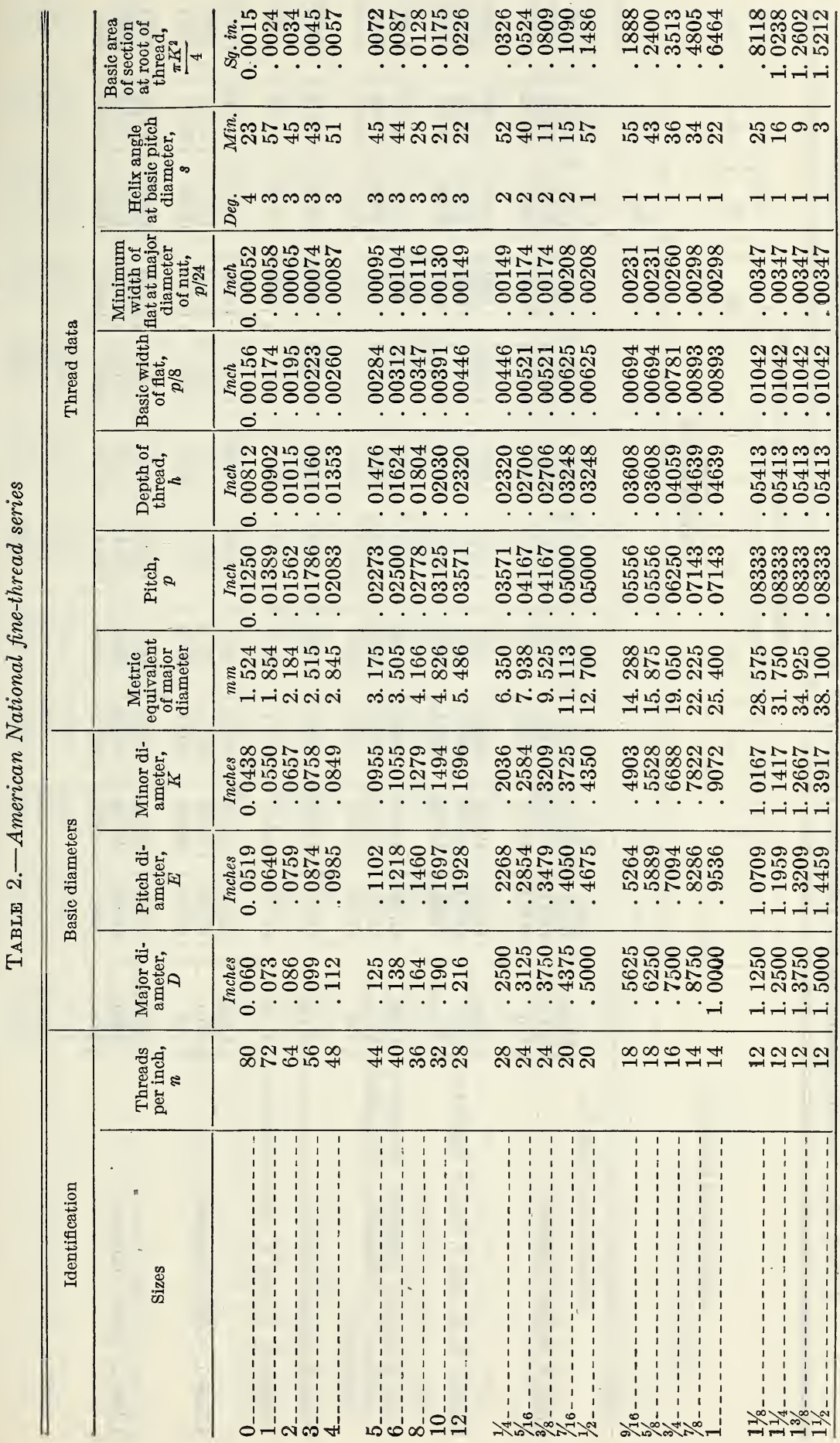




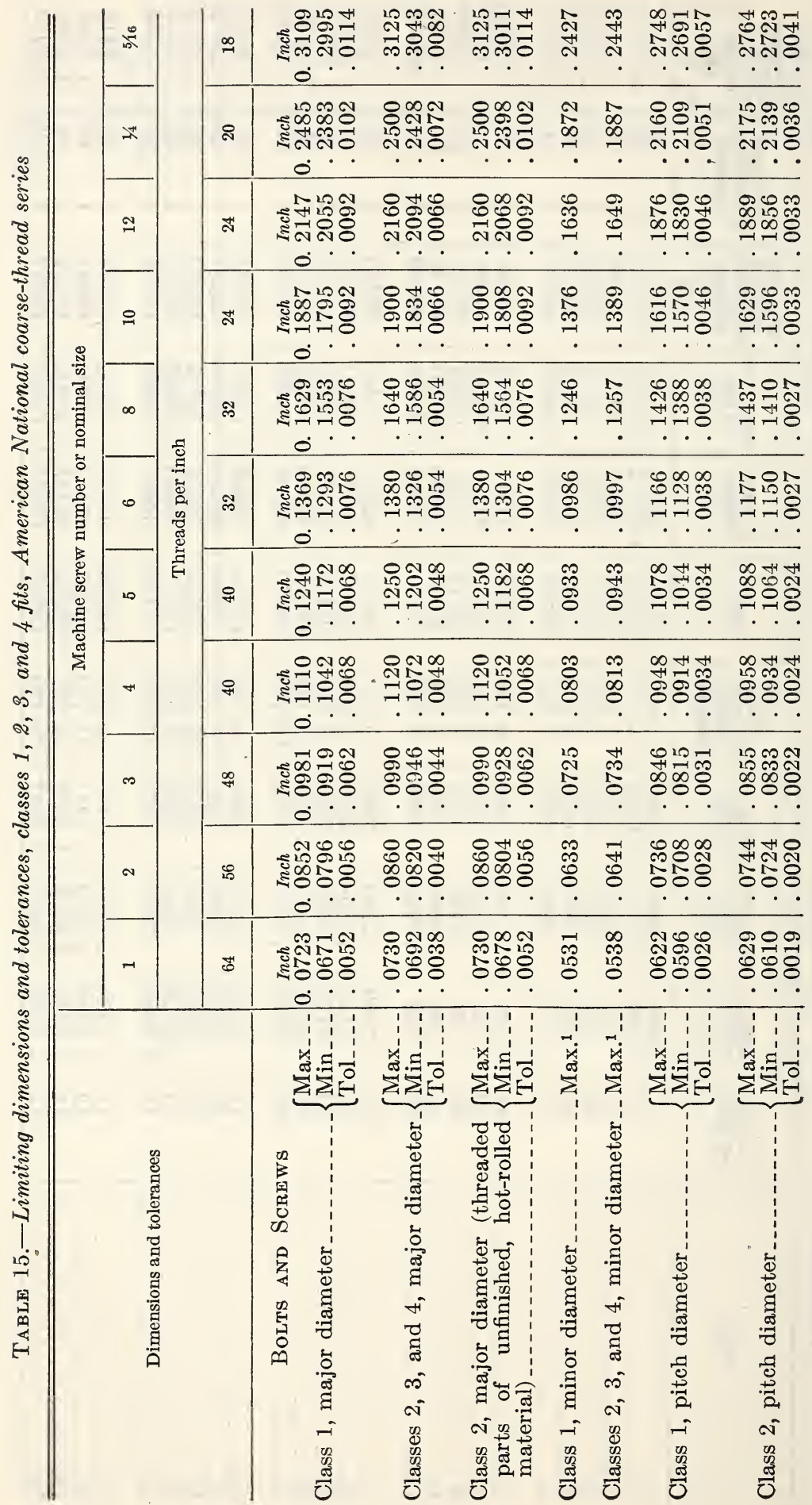


Screw Threads and Tap-Drill Sizes

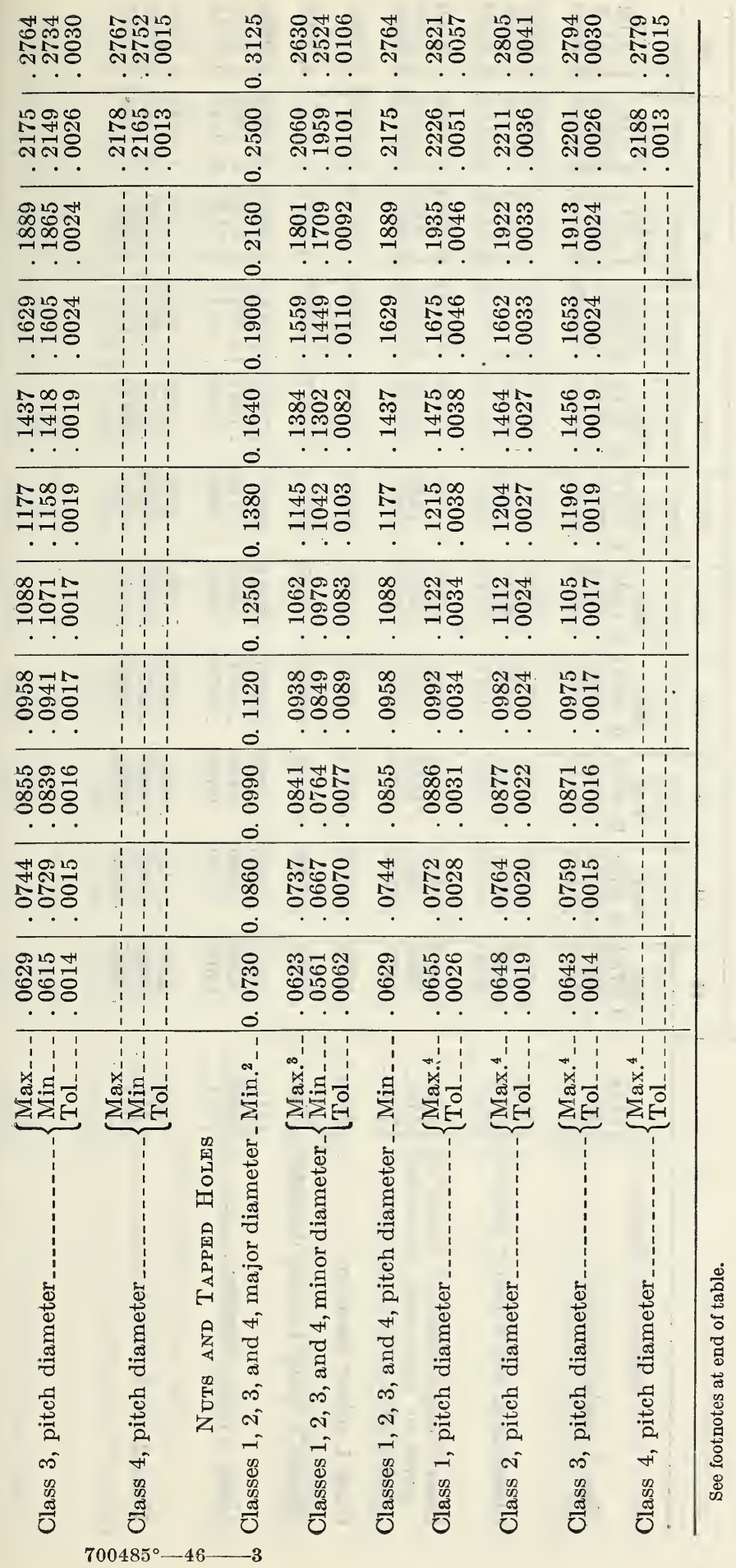




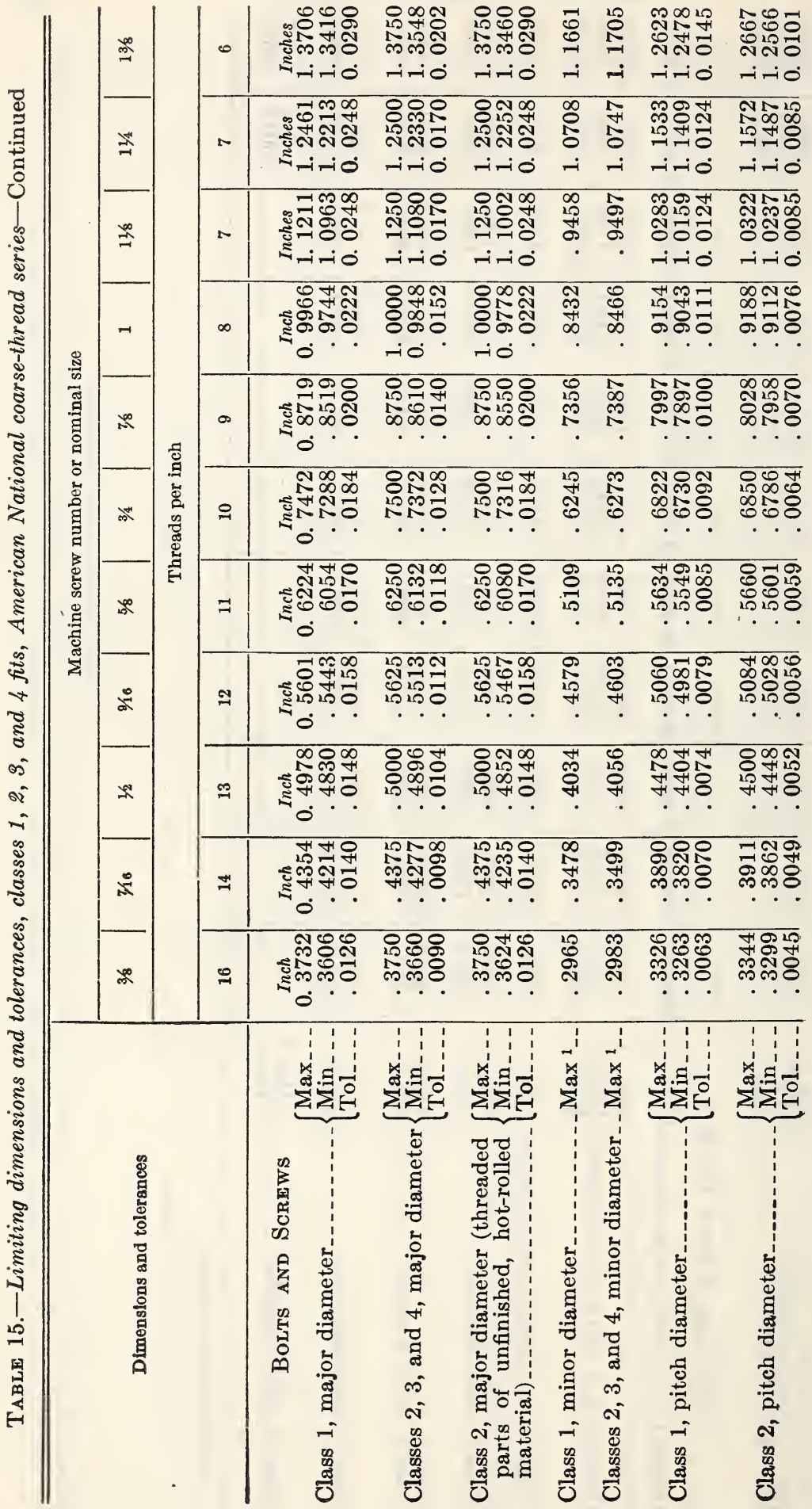




\begin{tabular}{|c|c|c|c|c|c|c|c|c|}
\hline 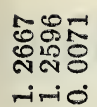 & 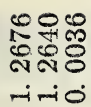 & 足 & 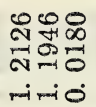 & 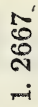 & 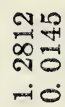 & 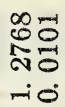 & 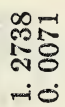 & $\begin{array}{l}\text { कृष } \\
\text { 슝 } \\
\text {-io } \\
\text {-io }\end{array}$ \\
\hline 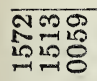 & $\begin{array}{l}80 \\
0100 \\
1920 \\
120\end{array}$ & $\begin{array}{l}8 \\
\text { ผ } \\
\text { ลิ }\end{array}$ & $\begin{array}{l}\infty .45 \\
0.5050 \\
=050\end{array}$ & $\underset{\substack{10 \\
10}}{\mathbb{2}}$ & 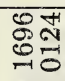 & $\begin{array}{l}120 \\
120 \\
08 \\
-10\end{array}$ & 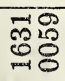 & 워요 \\
\hline -i -io & - - & -i & -i-io & $-i$ & -io & $-i 0^{\circ}$ & -10 & $-i 0$ \\
\hline 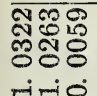 & 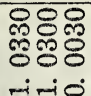 & $\begin{array}{l}\text { in } \\
\text { N } \\
\text { I }\end{array}$ & 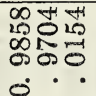 & $\begin{array}{l}\text { N } \\
\text { ซ్ } \\
-1\end{array}$ & 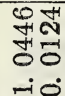 & $\begin{array}{l}10 \\
0 \% \\
\text { ㅇㅇㅇ } \\
\text {-i. }\end{array}$ & 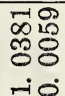 & 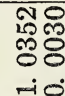 \\
\hline $\begin{array}{l}\infty \\
\infty \\
\infty \\
\infty \\
\infty \\
\sigma\end{array}$ & 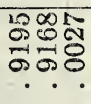 & 8 & 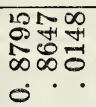 & $\begin{array}{l}\infty \\
\infty \\
\infty \\
-1\end{array}$ & $\begin{array}{l}\text { क्नेन } \\
\text { ฝैन }\end{array}$ & $\begin{array}{l}\text { to } \\
\text { ๙ै०ᄋ }\end{array}$ & 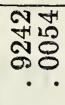 & \\
\hline
\end{tabular}

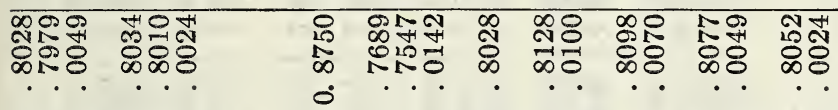

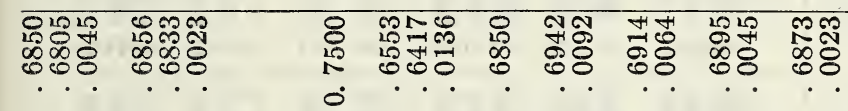

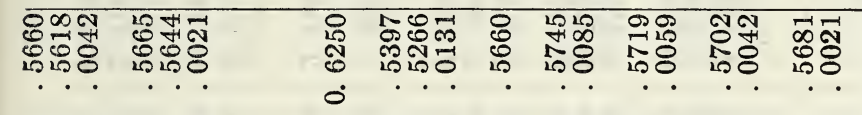

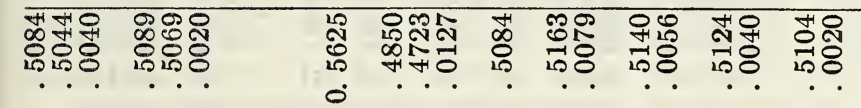

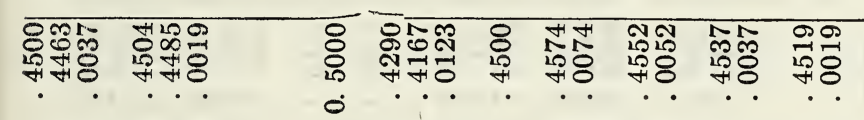

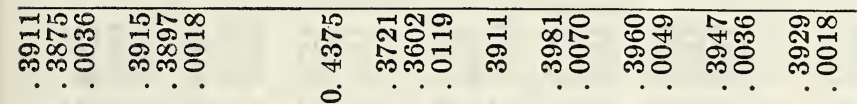

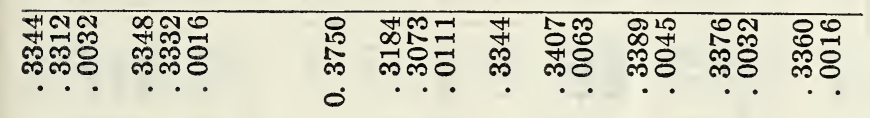

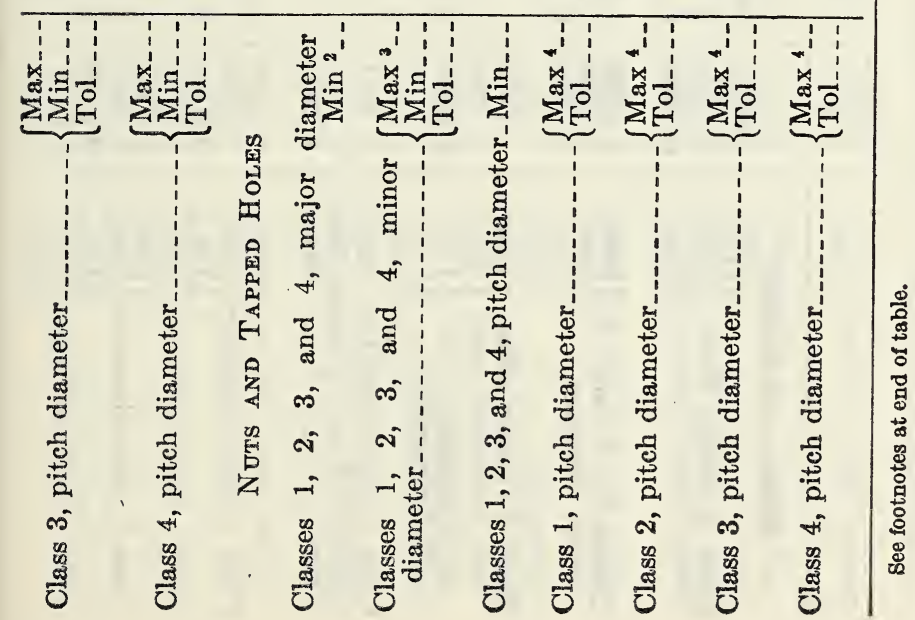




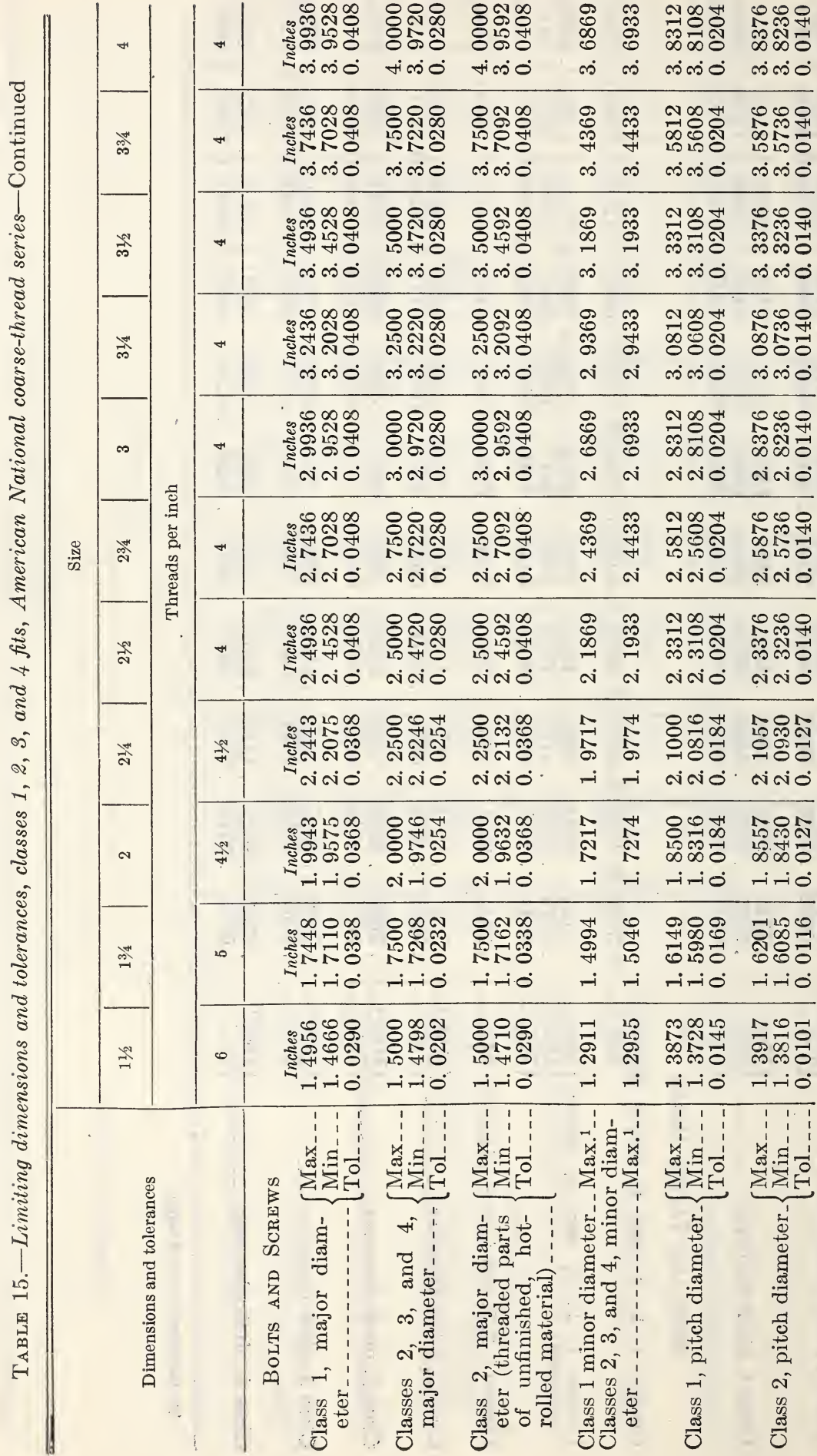




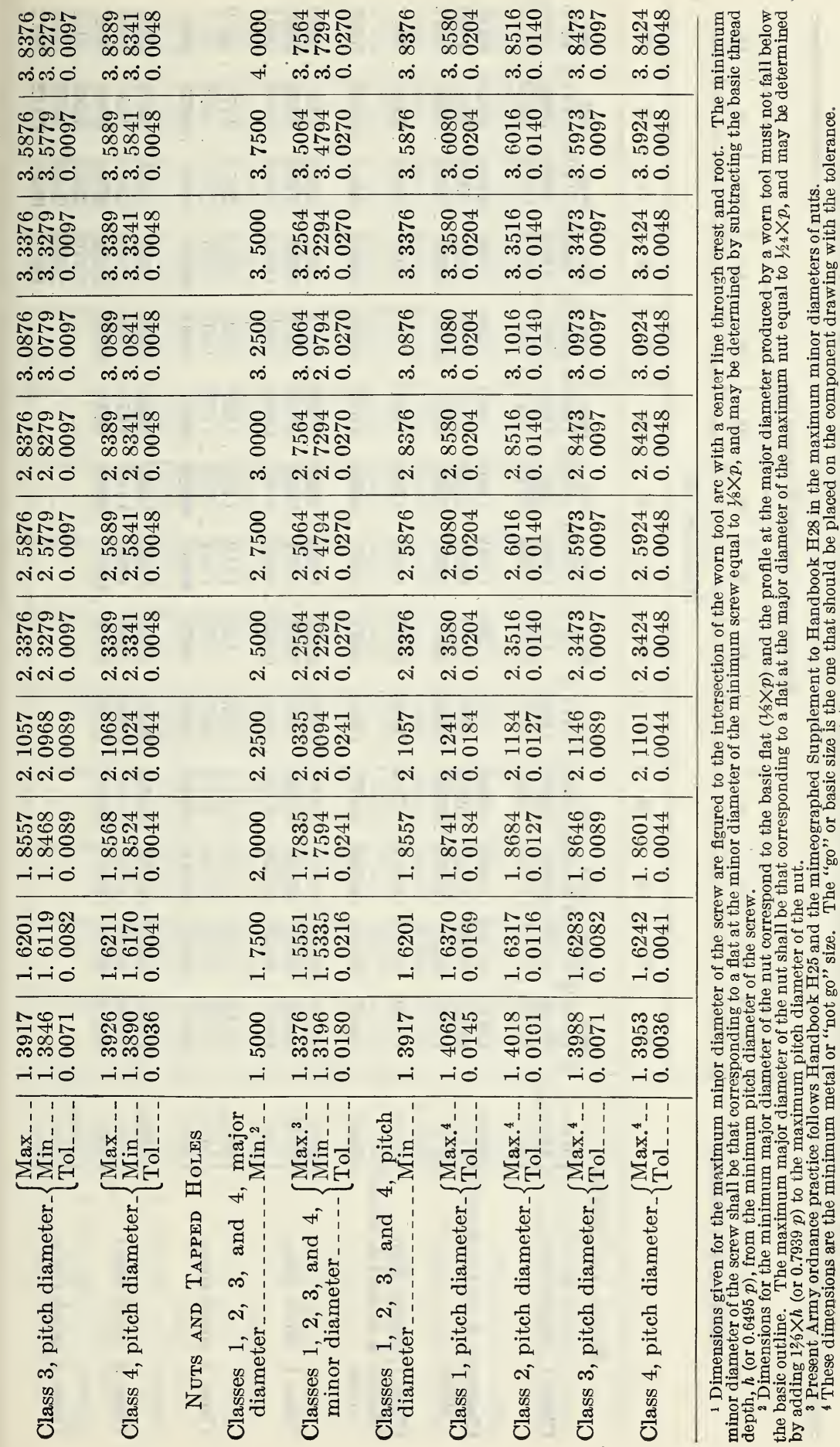




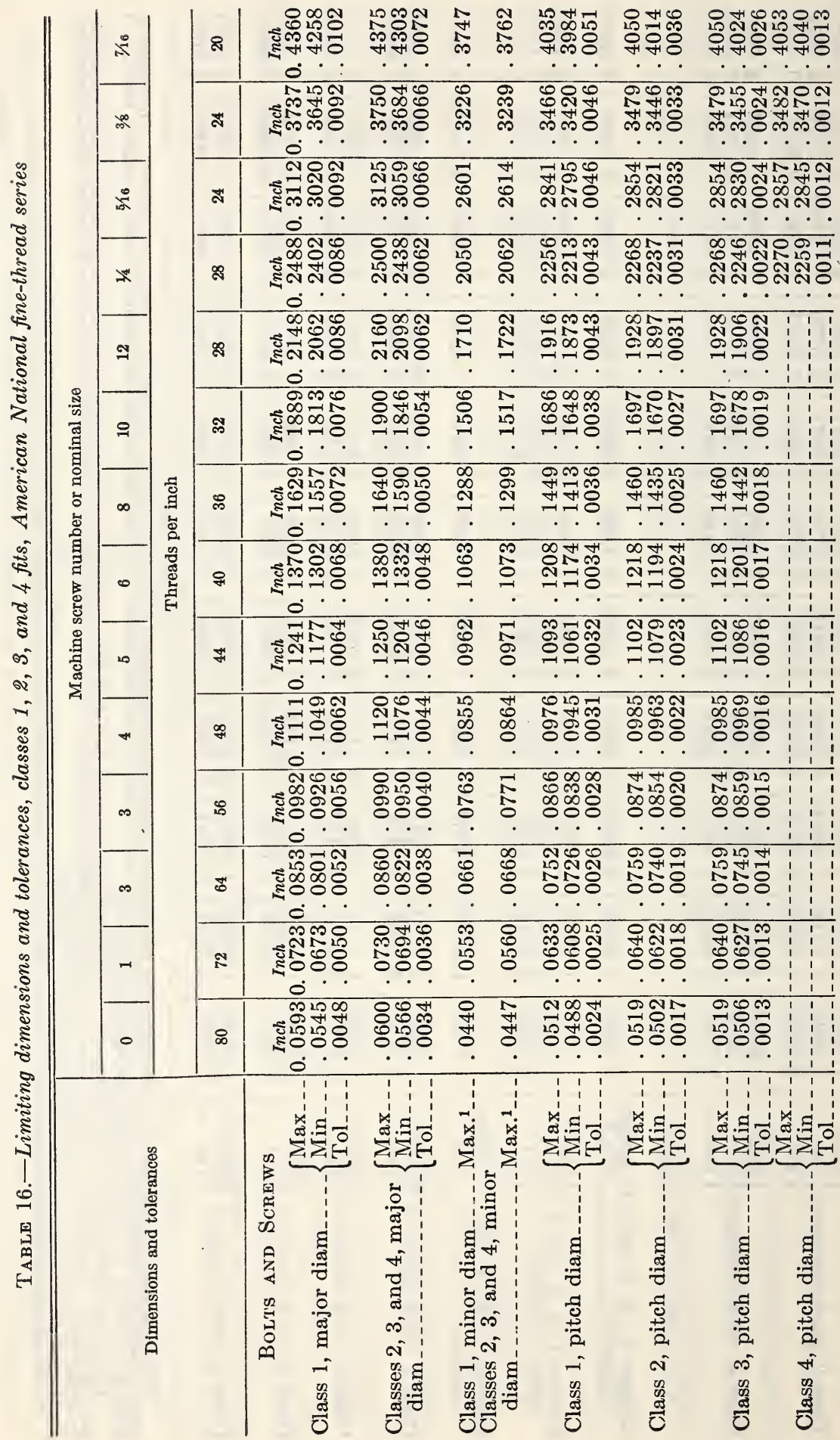




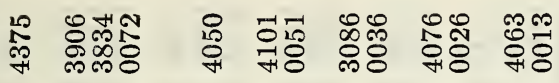

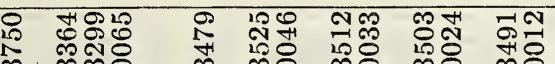

荌

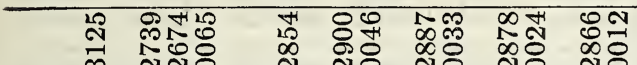

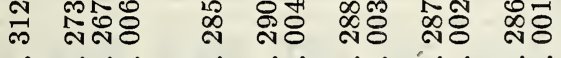

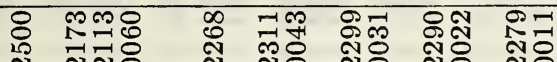

ฟ

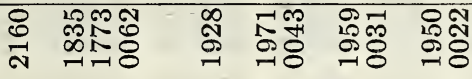

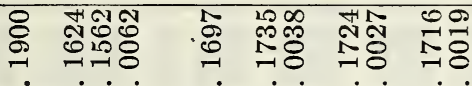

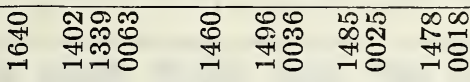

- 080 $\infty$ 재

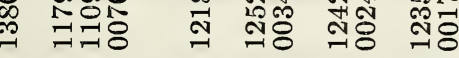

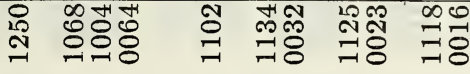

아용

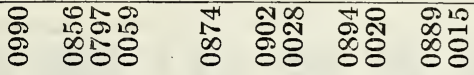

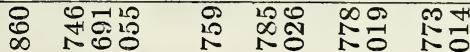

0 뇽요

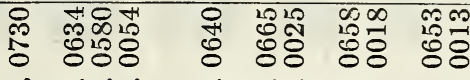

\&

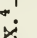

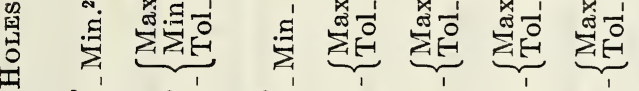

नि

चี चี

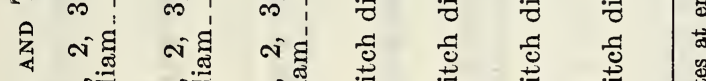

क تचี -

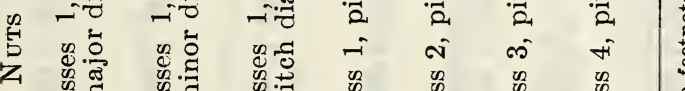

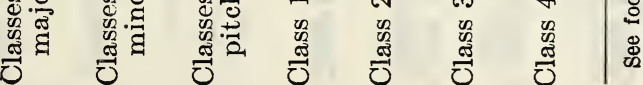




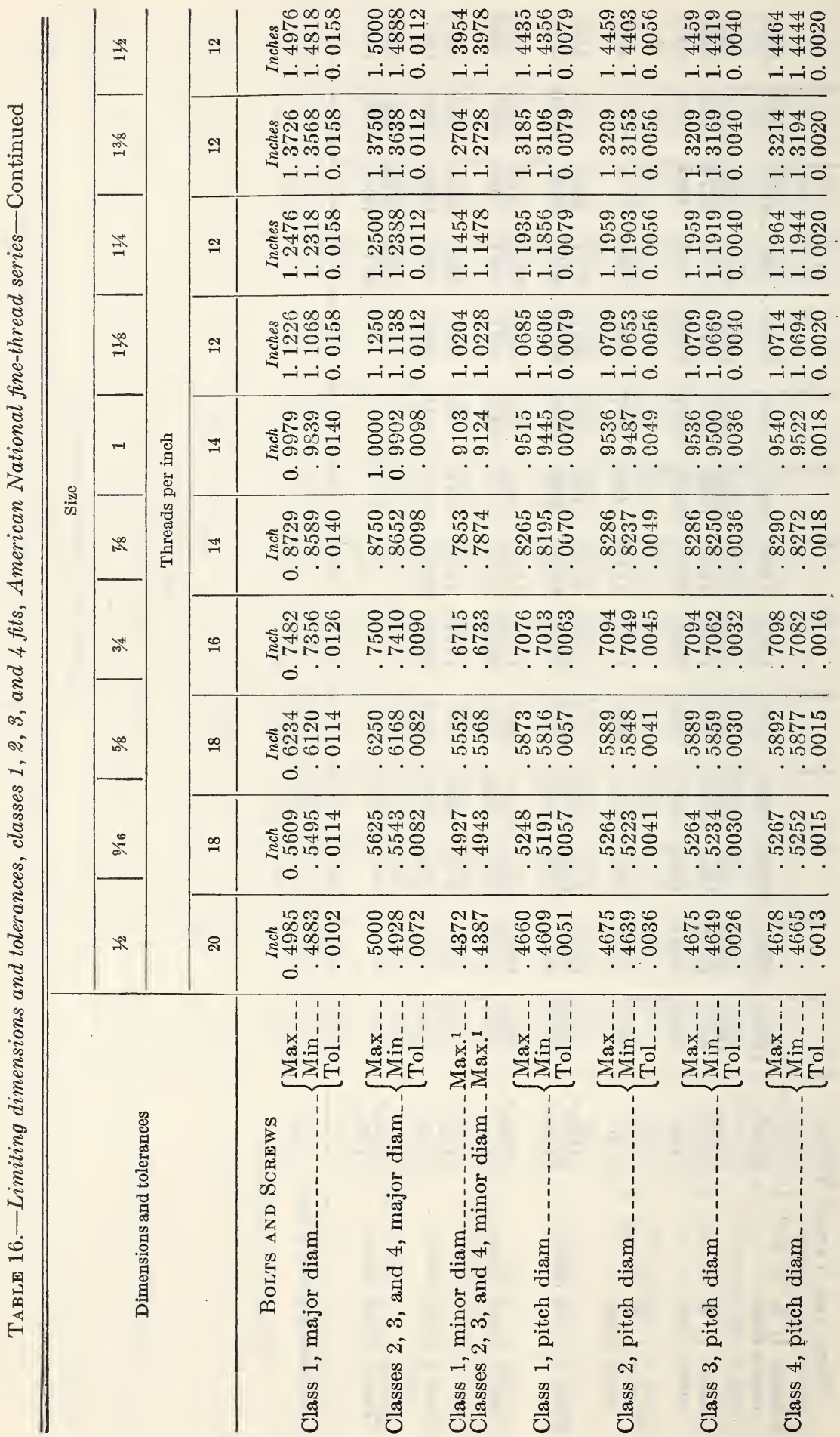




\begin{tabular}{|c|c|c|c|c|c|c|}
\hline $\begin{array}{l}8 \\
8 \\
8 \\
12 \\
-i\end{array}$ & 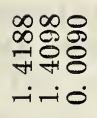 & $\begin{array}{l}8 \\
\text { in } \\
\text { म } \\
-1\end{array}$ & 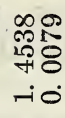 & $\begin{array}{l}200 \\
20 \\
2120 \\
710 \\
-i 0\end{array}$ & $\begin{array}{l}\text { 요여 } \\
\text { H요 } \\
\text {-io }\end{array}$ & $\begin{array}{l}\text { 요 } \\
\text { ㄱㅇㅇ } \\
\text {-io }\end{array}$ \\
\hline 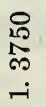 & 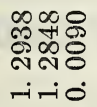 & $\begin{array}{l}\stackrel{\circ}{\circ} \\
\text { ๓ి } \\
-i\end{array}$ & $\begin{array}{l}\infty \\
\infty \\
\text { No } \\
\text { ஸి } \\
-i 0\end{array}$ & $\begin{array}{l}12 \% \\
012 \\
\text { No } \\
\text { - } \\
-10\end{array}$ & 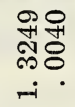 & 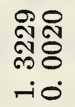 \\
\hline $\begin{array}{l}8 \\
\text { م } \\
\text { N } \\
-i\end{array}$ & $\begin{array}{l}\infty \\
\infty \\
\infty \\
0 \\
0 \\
-10 \\
\text {-i }\end{array}$ & $\begin{array}{l}28 \\
20 \\
2 \\
-1 \\
-1\end{array}$ & 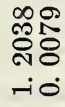 & $\begin{array}{l}120 \\
0.20 \\
\text { ㄱㅇㅇ } \\
\text {-io }\end{array}$ & $\begin{array}{l}\text { 웅ㅇ } \\
\text { \&용 } \\
\text {-io }\end{array}$ & $\begin{array}{l}\text { 유 } \\
\text { సరి } \\
-10 \\
\text {-io }\end{array}$ \\
\hline $\begin{array}{l}0 \\
\text { In } \\
\text { N1 } \\
\text {-i }\end{array}$ & 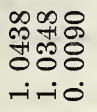 & $\begin{array}{l}8 \\
\stackrel{8}{5} \\
0 \\
-i\end{array}$ & $\begin{array}{l}\infty 0 \\
\infty \\
\infty 0 \\
08 \\
\text {-io }\end{array}$ & $\begin{array}{l}20 \% \\
020 \\
58 \\
-10\end{array}$ & $\begin{array}{l}\text { 웅위 } \\
\text { ㄷㅇㅇ } \\
\text {-io }\end{array}$ & $\begin{array}{l}\text { 융ำ } \\
\text { ஸेᄋ } \\
\text {-io }\end{array}$ \\
\hline $\begin{array}{l}8 \\
8 \\
8 \\
-1\end{array}$ & 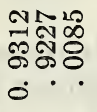 & $\begin{array}{l}0 \\
\text { : } \\
10 \\
\sigma\end{array}$ & $\begin{array}{l}\text { 융 } \\
\text { 용 }\end{array}$ & $\begin{array}{l}120 \\
100 \\
28 \\
0\end{array}$ & $\begin{array}{l}\text { No } \\
128 \\
58\end{array}$ & $\begin{array}{l}110 \\
250 \\
28 \\
0.9\end{array}$ \\
\hline
\end{tabular}

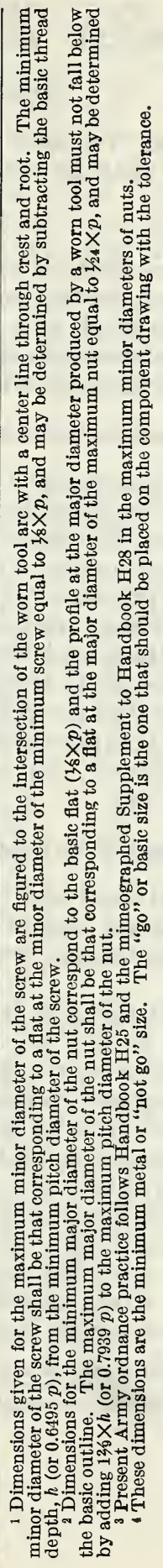

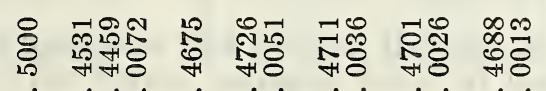

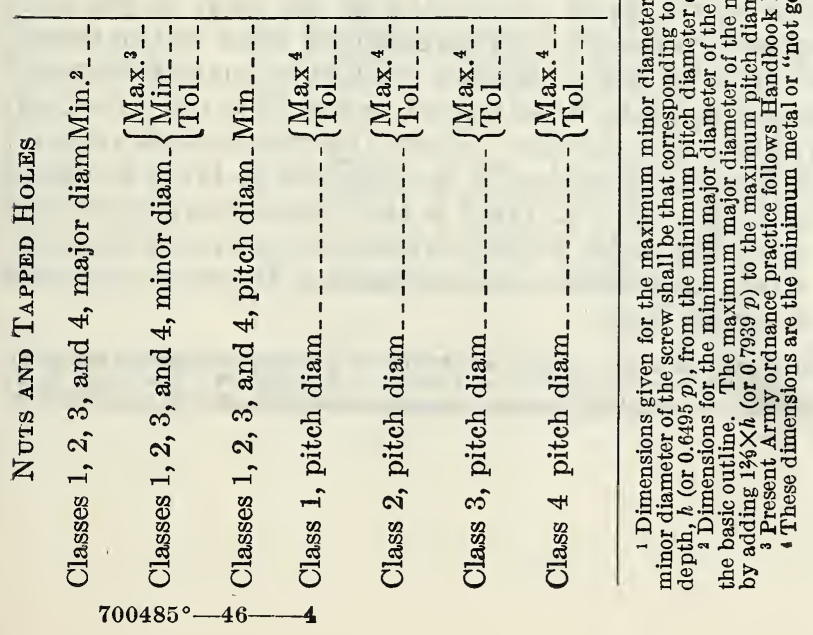


UNIFORM-PITCH SCREW-THREAD SERIES FOR HIGH-PRESSURE FASTENINGS, BOILER APPLICATIONS, MACHINERY COMPONENTS, ETC. ${ }^{3}$

FORM OF THREAD

9. The American National form of thread profile as specified in paragraphs 7 to $7 \mathrm{f}$ shall be used.

\section{THREAD SERIES}

9a. Where special threads are required, it is sometimes essential to select a certain pitch as standard for a range of sizes. Also, in general practice, where the pitch of a special thread is optional, the uniform use of a selected pitch is advantageous. For such applications 8,12 , and 16 threads per inch are widely used.

\section{AMERICAN NATIONAL 8-PITCH-THREAD SERIES}

9b. In table 26 are specified the nominal sizes and basic dimensions of the "American National 8-pitch-thread series." Limiting dimensions and tolerances for classes 2 and 3 fits are specified in table 29 .

Bolts for high-pressure pipe flanges, cylinder-head studs, and similar fastenings against pressure require that an initial tension be set up in the fastening, by elastic deformation of the fastening and the components held together, such that the joint will not open up when the steam or other pressure is applied. To secure a proper initial tension it is not practicable that the pitch should increase with the diameter of the thread, as the torque required to assemble the fastening would be excessive. Accordingly, for such purposes the 8-pitch thread has come into general use.

\section{AMERICAN NATIONAL 12-PITCH-THREAD SERIES}

9c. The nominal sizes and basic dimensions of the "American National 12-pitch-thread series" are specified in table 27. Limiting dimensions and tolerances for classes 2 and 3 fits are specified in table 30 .

Sizes of 12-pitch threads from one-half inch to and including one and three-fourths inches are used in boiler practice, which requires that worn stud holes be retapped with a tap of the next larger size, the increment being one-sixteenth inch throughout most of the range. Die-head chasers for sizes up to 3 inches are stocked by manufacturers. ${ }^{4}$

The 12-pitch threads are also widely used in machine construction, as for thin nuts on shafts and sleeves. From the standpoints of good design and simplification of practice, it is desirable to limit shoulder diameters to $1 / 8$-inch steps. The 12 pitch is the coarsest in general use that will permit a threaded collar which screws onto a threaded shoulder to slip over a shaft, the difference in diameter between shoulder and shaft being one-eighth inch.

\footnotetext{
3 This standard, in substantially the same form, has been adopted by the American Standards Association. It is published as ASA B1.1-1935 "Screw Threads" by the ASME, 29 West 39th St., New York, N. Y. "See U. S. Department of Commerce Simplified Practice Recommendation R51-29, Die Head Chasers.
} 


\section{AMERICAN NATIONAL 16-PITCH-THREAD SERIES}

9d. The nominal sizes and basic dimensions of the "American National 16-pitch-thread series" are specified in table 28. Limiting dimensions and tolerances for classes 2 and 3 fits are specified in table 31.

The 16-pitch series is a uniform pitch series for such applications as require a relatively fine thread. It is intended primarily for use on threaded adjusting collars and bearing-retaining nuts.

\section{TABLE 26.-American National 8-pitch thread series}

[Pitch, $p=0.12500$ inch; depth of thread, $h=0.08119$ inch; basic width of flat, $p / 8=0.01562$ inch; minimum width of flat at major diameter of nut, $p / 24=0.00521$ inch.]

\begin{tabular}{|c|c|c|c|c|c|c|c|c|}
\hline \multicolumn{2}{|c|}{ Identification } & \multicolumn{3}{|c|}{ Basic diameters } & \multicolumn{4}{|c|}{ Thread data } \\
\hline Sizes & $\begin{array}{l}\text { Threads } \\
\text { per inch }\end{array}$ & $\begin{array}{c}\text { Major } \\
\text { diameter, } \\
D\end{array}$ & $\begin{array}{c}\text { Pitch } \\
\text { diameter, } \\
E\end{array}$ & $\begin{array}{c}\text { Minor } \\
\text { diameter, } \\
\underset{K}{\text { dine }}\end{array}$ & $\begin{array}{l}\text { Metric } \\
\text { equivalent } \\
\text { of major } \\
\text { diameter }\end{array}$ & $\begin{array}{r}\text { Heli } \\
\text { at } \\
\text { p } \\
\text { diar } \\
s\end{array}$ & $\begin{array}{l}\text { angle } \\
\text { asic } \\
\text { ch } \\
\text { eter, }\end{array}$ & $\begin{array}{c}\text { Basic area } \\
\text { of section } \\
\text { at root of } \\
\text { thread, } \\
\frac{\pi K^{2}}{4}\end{array}$ \\
\hline $\begin{array}{r}\text { Inches } \\
111 / 8- \\
11 / 4 \ldots \\
13 / 8 \\
11 / 2 \ldots \\
\end{array}$ & $\begin{array}{l}8 \\
8 \\
8 \\
8 \\
8\end{array}$ & $\begin{array}{l}\text { Inches } \\
1.0000 \\
1.1250 \\
1.2500 \\
1.3750 \\
1.5000\end{array}$ & $\begin{array}{l}\text { Inches } \\
0.9188 \\
1.0438 \\
1.1688 \\
1.2938 \\
1.4188\end{array}$ & $\begin{array}{c}\text { Inches } \\
0.8376 \\
.9626 \\
1.0876 \\
1.2126 \\
1.3376\end{array}$ & $\begin{array}{l}\text { mm } \\
\text { 25. } 400 \\
28.575 \\
31.750 \\
34.925 \\
38.100\end{array}$ & $\begin{array}{l}\text { deg } \\
2 \\
2 \\
1 \\
1 \\
1\end{array}$ & $\begin{array}{r}\min \\
29 \\
11 \\
57 \\
46 \\
36\end{array}$ & $\begin{array}{l}\text { Square } \\
\text { inches } \\
0.5510 \\
.7277 \\
.9290 \\
1.1548 \\
1.4052\end{array}$ \\
\hline $\begin{array}{l}15 / 8 \ldots- \\
13 / 4 \ldots- \\
17 / 8 \ldots- \\
2 \\
21 / 8 \ldots\end{array}$ & $\begin{array}{l}8 \\
8 \\
8 \\
8 \\
8\end{array}$ & $\begin{array}{l}\text { 1. } 6250 \\
\text { 1. } 7500 \\
\text { 1. } 8750 \\
\text { 2. } 0000 \\
\text { 2. } 1250\end{array}$ & $\begin{array}{l}\text { 1. } 5438 \\
\text { 1. } 6688 \\
\text { 1. } 7938 \\
\text { 1. } 9188 \\
\text { 2. } 0438\end{array}$ & $\begin{array}{l}\text { 1. } 4626 \\
\text { 1. } 5876 \\
1.7126 \\
\text { 1. } 8376 \\
1.9626\end{array}$ & $\begin{array}{l}41.275 \\
44.450 \\
47.625 \\
50.800 \\
53.975\end{array}$ & $\begin{array}{l}1 \\
1 \\
1 \\
1 \\
1\end{array}$ & $\begin{array}{r}29 \\
22 \\
16 \\
11 \\
7\end{array}$ & $\begin{array}{l}\text { 1. } 6801 \\
\text { 1. } 9796 \\
\text { 2. } 3036 \\
\text { 2. } 6521 \\
\text { 3. } 0252\end{array}$ \\
\hline $\begin{array}{l}21 / 4-\ldots \\
21 / 2-- \\
23 / 4-- \\
3- \\
31 / 4 \ldots-\end{array}$ & $\begin{array}{l}8 \\
8 \\
8 \\
8 \\
8\end{array}$ & $\begin{array}{l}\text { 2. } 2500 \\
\text { 2. } 5000 \\
\text { 2. } 7500 \\
\text { 3. } 0000 \\
\text { 3. } 2500\end{array}$ & $\begin{array}{l}\text { 2. } 1688 \\
\text { 2. } 4188 \\
\text { 2. } 6688 \\
\text { 2. } 9188 \\
\text { 3. } 1688\end{array}$ & $\begin{array}{l}\text { 2. } 0876 \\
\text { 2. } 3376 \\
\text { 2. } 5876 \\
\text { 2. } 8376 \\
\text { 3. } 0876\end{array}$ & $\begin{array}{l}\text { 57. } 150 \\
63.500 \\
69.850 \\
76.200 \\
82.550\end{array}$ & $\begin{array}{l}1 \\
0 \\
0 \\
0 \\
0\end{array}$ & $\begin{array}{l}3 \\
57 \\
51 \\
47 \\
43\end{array}$ & $\begin{array}{l}\text { 3. } 4228 \\
\text { 4. } 2917 \\
\text { 5. } 2588 \\
\text { 6. } 3240 \\
\text { 7. } 4874\end{array}$ \\
\hline 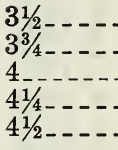 & $\begin{array}{l}8 \\
8 \\
8 \\
8 \\
8\end{array}$ & $\begin{array}{l}\text { 3. } 5000 \\
\text { 3. } 7500 \\
\text { 4. } 0000 \\
\text { 4. } 2500 \\
\text { 4. } 5000\end{array}$ & $\begin{array}{l}\text { 3. } 4188 \\
\text { 3. } 6688 \\
\text { 3. } 9188 \\
\text { 4. } 1688 \\
\text { 4. } 4188\end{array}$ & $\begin{array}{l}\text { 3. } 3376 \\
\text { 3. } 5876 \\
\text { 3. } 8376 \\
\text { 4. } 0876 \\
\text { 4. } 3376\end{array}$ & $\begin{array}{r}88.900 \\
95.250 \\
101.600 \\
107.950 \\
114.300\end{array}$ & $\begin{array}{l}0 \\
0 \\
0 \\
0 \\
0\end{array}$ & $\begin{array}{l}40 \\
37 \\
35 \\
33 \\
31\end{array}$ & $\begin{array}{r}\text { 8. } 7490 \\
10.1088 \\
11.5667 \\
13.1228 \\
14.7771\end{array}$ \\
\hline $\begin{array}{l}43 / 4-- \\
5 \\
51 / 4- \\
51 / 2 \\
534\end{array}$ & $\begin{array}{l}8 \\
8 \\
8 \\
8 \\
8\end{array}$ & $\begin{array}{l}\text { 4. } 7500 \\
\text { 5. } 0000 \\
\text { 5. } 2500 \\
\text { 5. } 5000 \\
\text { 5. } 7500\end{array}$ & $\begin{array}{l}\text { 4. } 6688 \\
\text { 4. } 9188 \\
\text { 5. } 1688 \\
\text { 5. } 4188 \\
\text { 5. } 6688\end{array}$ & $\begin{array}{l}\text { 4. } 5876 \\
\text { 4. } 8376 \\
\text { 5. } 0876 \\
\text { 5. } 3376 \\
\text { 5. } 5876\end{array}$ & $\begin{array}{l}120.650 \\
127.000 \\
133.350 \\
139.700 \\
146.050\end{array}$ & $\begin{array}{l}0 \\
0 \\
0 \\
0 \\
0\end{array}$ & $\begin{array}{l}29 \\
28 \\
26 \\
25 \\
24\end{array}$ & $\begin{array}{l}\text { 16. } 5295 \\
\text { 18. } 3802 \\
20.3290 \\
22.3760 \\
24.5211\end{array}$ \\
\hline $6 \ldots$ & 8 & 6. 0000 & 5. 9188 & 5. 8376 & 152. 400 & 0 & 23 & 26. 7645 \\
\hline
\end{tabular}

1 Standard size of the American National coarse-thread series. 
TABle 27.-American National 12-pitch thread series

[Pitch, $p=0.08333$ inch; depth of thread, $h=0.05413$ inch; basic width of flat, $p / 8=0.01042$ inch; minimum width of flat at major diameter of nut, $p / 24=0.00347$ inch.]

\begin{tabular}{|c|c|c|c|c|c|c|c|c|}
\hline \multicolumn{2}{|c|}{ Identification } & \multicolumn{3}{|c|}{ Basic diameters } & \multicolumn{4}{|c|}{ Thread data } \\
\hline Sizes & $\begin{array}{l}\text { Threads } \\
\text { per inch }\end{array}$ & $\begin{array}{c}\text { Major } \\
\text { diameter, } \\
D\end{array}$ & $\underset{E}{\text { Pitch }} \underset{\underset{E}{\text { diameter, }}}{ }$ & $\begin{array}{c}\text { Minor } \\
\text { diameter, } \\
K\end{array}$ & $\begin{array}{l}\text { Metric } \\
\text { equivalent } \\
\text { of major } \\
\text { diameter }\end{array}$ & $\begin{array}{r}\text { Helix } \\
\text { at } \\
\text { pi } \\
\text { dian }\end{array}$ & $\begin{array}{l}\text { angle } \\
\text { asic } \\
\text { tch } \\
\text { acter, } \\
8\end{array}$ & $\begin{array}{c}\text { Basic area } \\
\text { of section } \\
\text { at root of } \\
\text { thread, } \\
\frac{\pi K^{2}}{4}\end{array}$ \\
\hline 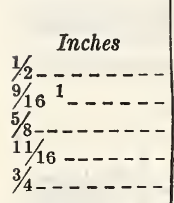 & $\begin{array}{l}12 \\
12 \\
12 \\
12 \\
12\end{array}$ & $\begin{array}{l}\text { Inches } \\
0.5000 \\
.5625 \\
.6250 \\
.6875 \\
.7500\end{array}$ & $\begin{array}{c}\text { Inches } \\
0.4459 \\
.5084 \\
.5709 \\
.6334 \\
.6959\end{array}$ & $\begin{array}{c}\text { Inches } \\
0.3917 \\
.4542 \\
.5167 \\
.5792 \\
.6417\end{array}$ & $\begin{array}{l}\text { mm } \\
\text { 12. } 700 \\
14.288 \\
15.875 \\
17.463 \\
19.050\end{array}$ & $\begin{array}{l}d e g \\
3 \\
2 \\
2 \\
2 \\
2\end{array}$ & $\begin{array}{l}\min \\
24 \\
59 \\
40 \\
24 \\
11\end{array}$ & $\begin{array}{l}\text { Square } \\
\text { inches } \\
0.1205 \\
.1620 \\
.2097 \\
.2635 \\
.3234\end{array}$ \\
\hline 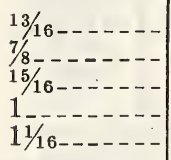 & $\begin{array}{l}12 \\
12 \\
12 \\
12 \\
12\end{array}$ & $\begin{array}{r}.8125 \\
.8750 \\
.9375 \\
1.0000 \\
1.0625\end{array}$ & $\begin{array}{r}.7584 \\
.8209 \\
.8834 \\
.9459 \\
1.0084\end{array}$ & $\begin{array}{l}.7042 \\
.7667 \\
.8292 \\
.8917 \\
.9542\end{array}$ & $\begin{array}{l}\text { 20. } 638 \\
22.225 \\
23.813 \\
25.400 \\
26.988\end{array}$ & $\begin{array}{l}2 \\
1 \\
1 \\
1 \\
1\end{array}$ & $\begin{array}{r}0 \\
51 \\
43 \\
36 \\
30\end{array}$ & $\begin{array}{l}.3895 \\
.4617 \\
.5400 \\
.6245 \\
.7151\end{array}$ \\
\hline $\begin{array}{l}11 / 8^{2}-\ldots- \\
13 / 16 \ldots- \\
11 / 4^{2}-\ldots-\cdots \\
15 / 16-\cdots \\
13 / 8^{2}-\ldots-\cdots\end{array}$ & $\begin{array}{l}12 \\
12 \\
12 \\
12 \\
12\end{array}$ & $\begin{array}{l}\text { 1. } 1250 \\
\text { 1. } 1875 \\
\text { 1. } 2500 \\
\text { 1. } 3125 \\
\text { 1. } 3750\end{array}$ & $\begin{array}{l}\text { 1. } 0709 \\
\text { 1. } 1334 \\
\text { 1. } 1959 \\
\text { 1. } 2584 \\
\text { 1. } 3209\end{array}$ & $\begin{array}{l}\text { 1. } 0167 \\
\text { 1. } 0792 \\
\text { 1. } 1417 \\
\text { 1. } 2042 \\
\text { 1. } 2667\end{array}$ & $\begin{array}{l}28.575 \\
30.163 \\
31.750 \\
33.338 \\
34.925\end{array}$ & $\begin{array}{l}1 \\
1 \\
1 \\
1 \\
1\end{array}$ & $\begin{array}{r}25 \\
20 \\
16 \\
12 \\
9\end{array}$ & $\begin{array}{r}.8118 \\
.9147 \\
1.0237 \\
1.1389 \\
1.2602\end{array}$ \\
\hline 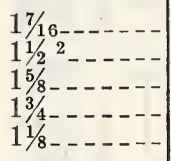 & $\begin{array}{l}12 \\
12 \\
12 \\
12 \\
12\end{array}$ & $\begin{array}{l}\text { 1. } 4375 \\
\text { 1. } 5000 \\
\text { 1. } 6250 \\
\text { 1. } 7500 \\
\text { 1. } 8750\end{array}$ & $\begin{array}{l}\text { 1. } 3834 \\
\text { 1. } 4459 \\
\text { 1. } 5709 \\
\text { 1. } 6959 \\
\text { 1. } 8209\end{array}$ & $\begin{array}{l}\text { 1. } 3292 \\
\text { 1. } 3917 \\
\text { 1. } 5167 \\
\text { 1. } 6417 \\
\text { 1. } 7667\end{array}$ & $\begin{array}{l}36.513 \\
38.100 \\
41.275 \\
44.450 \\
47.625\end{array}$ & $\begin{array}{l}1 \\
1 \\
0 \\
0 \\
0\end{array}$ & $\begin{array}{r}6 \\
3 \\
58 \\
54 \\
50\end{array}$ & $\begin{array}{l}\text { 1. } 3876 \\
\text { 1. } 5212 \\
\text { 1. } 8067 \\
\text { 2. } 1168 \\
\text { 2. } 4514\end{array}$ \\
\hline 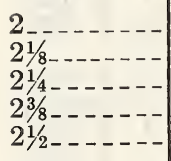 & $\begin{array}{l}12 \\
12 \\
12 \\
12 \\
12\end{array}$ & $\begin{array}{l}\text { 2. } 0000 \\
\text { 2. } 1250 \\
\text { 2. } 2500 \\
\text { 2. } 3750 \\
\text { 2. } 5000\end{array}$ & $\begin{array}{l}\text { 1. } 9459 \\
\text { 2. } 0709 \\
\text { 2. } 1959 \\
\text { 2. } 3209 \\
\text { 2. } 4459\end{array}$ & $\begin{array}{l}\text { 1. } 8917 \\
\text { 2. } 0167 \\
\text { 2. } 1417 \\
\text { 2. } 2667 \\
\text { 2. } 3917\end{array}$ & $\begin{array}{l}50.800 \\
53.975 \\
57.150 \\
60.325 \\
63.500\end{array}$ & $\begin{array}{l}0 \\
0 \\
0 \\
0 \\
0\end{array}$ & $\begin{array}{l}47 \\
44 \\
42 \\
39 \\
37\end{array}$ & $\begin{array}{l}\text { 2. } 8106 \\
\text { 3. } 1943 \\
\text { 3. } 6025 \\
\text { 4. } 0353 \\
\text { 4. } 4927\end{array}$ \\
\hline 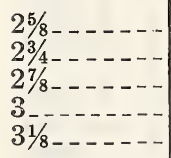 & $\begin{array}{l}12 \\
12 \\
12 \\
12 \\
12\end{array}$ & $\begin{array}{l}\text { 2. } 6250 \\
\text { 2. } 7500 \\
\text { 2. } 8750 \\
\text { 3. } 0000 \\
\text { 3. } 1250\end{array}$ & $\begin{array}{l}\text { 2. } 5709 \\
\text { 2. } 6959 \\
\text { 2. } 8209 \\
\text { 2. } 9459 \\
\text { 3. } 0709\end{array}$ & $\begin{array}{l}\text { 2. } 5167 \\
\text { 2. } 6417 \\
\text { 2. } 7667 \\
\text { 2. } 8917 \\
\text { 3. } 0167\end{array}$ & & $\begin{array}{l}0 \\
0 \\
0 \\
0 \\
0\end{array}$ & $\begin{array}{l}35 \\
34 \\
32 \\
31 \\
30\end{array}$ & $\begin{array}{l}\text { 4. } 9745 \\
\text { 5. } 4810 \\
6.0119 \\
6.5674 \\
\text { 7. } 1475\end{array}$ \\
\hline 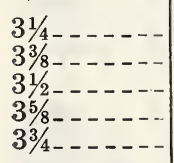 & $\begin{array}{l}12 \\
12 \\
12 \\
12 \\
12\end{array}$ & $\begin{array}{l}\text { 3. } 2500 \\
\text { 3. } 3750 \\
\text { 3. } 5000 \\
\text { 3. } 6250 \\
\text { 3. } 7500\end{array}$ & $\begin{array}{l}\text { 3. } 1959 \\
\text { 3. } 3209 \\
\text { 3. } 4459 \\
\text { 3. } 5709 \\
\text { 3. } 6959\end{array}$ & $\begin{array}{l}\text { 3. } 1417 \\
\text { 3. } 2667 \\
\text { 3. } 3917 \\
\text { 3. } 5167 \\
\text { 3. } 6417\end{array}$ & & $\begin{array}{l}0 \\
0 \\
0 \\
0 \\
0\end{array}$ & $\begin{array}{l}29 \\
27 \\
26 \\
26 \\
25\end{array}$ & $\begin{array}{r}\text { 7. } 7521 \\
8.3812 \\
9.0349 \\
\text { 9. } 7132 \\
\text { 10. } 4159\end{array}$ \\
\hline 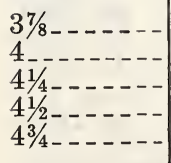 & $\begin{array}{l}12 \\
12 \\
12 \\
12 \\
12\end{array}$ & $\begin{array}{l}\text { 3. } 8750 \\
\text { 4. } 0000 \\
\text { 4. } 2500 \\
\text { 4. } 5000 \\
\text { 4. } 7500\end{array}$ & $\begin{array}{l}\text { 3. } 8209 \\
\text { 3. } 9459 \\
\text { 4. } 1959 \\
\text { 4. } 4459 \\
\text { 4. } 6959\end{array}$ & $\begin{array}{l}\text { 3. } 7667 \\
\text { 3. } 8917 \\
\text { 4. } 1417 \\
\text { 4. } 3917 \\
\text { 4. } 6417\end{array}$ & $\begin{array}{r}98.425 \\
101.600 \\
107.950 \\
114.300 \\
120.650\end{array}$ & $\begin{array}{l}0 \\
0 \\
0 \\
0 \\
0\end{array}$ & $\begin{array}{l}24 \\
23 \\
22 \\
21 \\
19\end{array}$ & $\begin{array}{l}\text { 11. } 1433 \\
11.8951 \\
\text { 13. } 4725 \\
\text { 15. } 1480 \\
16.9217\end{array}$ \\
\hline $\begin{array}{l}5 \\
5114 \ldots \\
51 / 2 \ldots \\
53 / 4-\ldots-\cdots \\
6 \ldots-\ldots\end{array}$ & $\begin{array}{l}12 \\
12 \\
12 \\
12 \\
12\end{array}$ & $\begin{array}{l}\text { 5. } 0000 \\
\text { 5. } 2500 \\
\text { 5. } 5000 \\
\text { 5. } 7500 \\
\text { 6. } 0000\end{array}$ & $\begin{array}{l}\text { 4. } 9459 \\
\text { 5. } 1959 \\
\text { 5. } 4459 \\
\text { 5. } 6959 \\
\text { 5. } 9459\end{array}$ & $\begin{array}{l}\text { 4. } 8917 \\
\text { 5. } 1417 \\
\text { 5. } 3917 \\
\text { 5. } 6417 \\
\text { 5. } 8917\end{array}$ & $\begin{array}{l}\text { 127. } 000 \\
133.350 \\
139.700 \\
146.050 \\
152.400\end{array}$ & $\begin{array}{l}0 \\
0 \\
0 \\
0 \\
0\end{array}$ & $\begin{array}{l}18 \\
18 \\
17 \\
16 \\
15\end{array}$ & $\begin{array}{l}\text { 18. } 7936 \\
\text { 20. } 7636 \\
\text { 22. } 8319 \\
\text { 24. } 9983 \\
\text { 27. } 2628\end{array}$ \\
\hline
\end{tabular}

1 Standard size of the American National coarse-thread series.

2 Standard size of the American National fine-thread series. 
TABLE 28.-American National 16-pitch thread series

[Pitch, $p=0.06250$ inch; depth of thread, $h=0.04059$ inch; basic width of flat, $p / 8=0.00781$ inch; minimum width of flat at major diameter of nut, $p / 24=0.00260 \mathrm{inch}]$

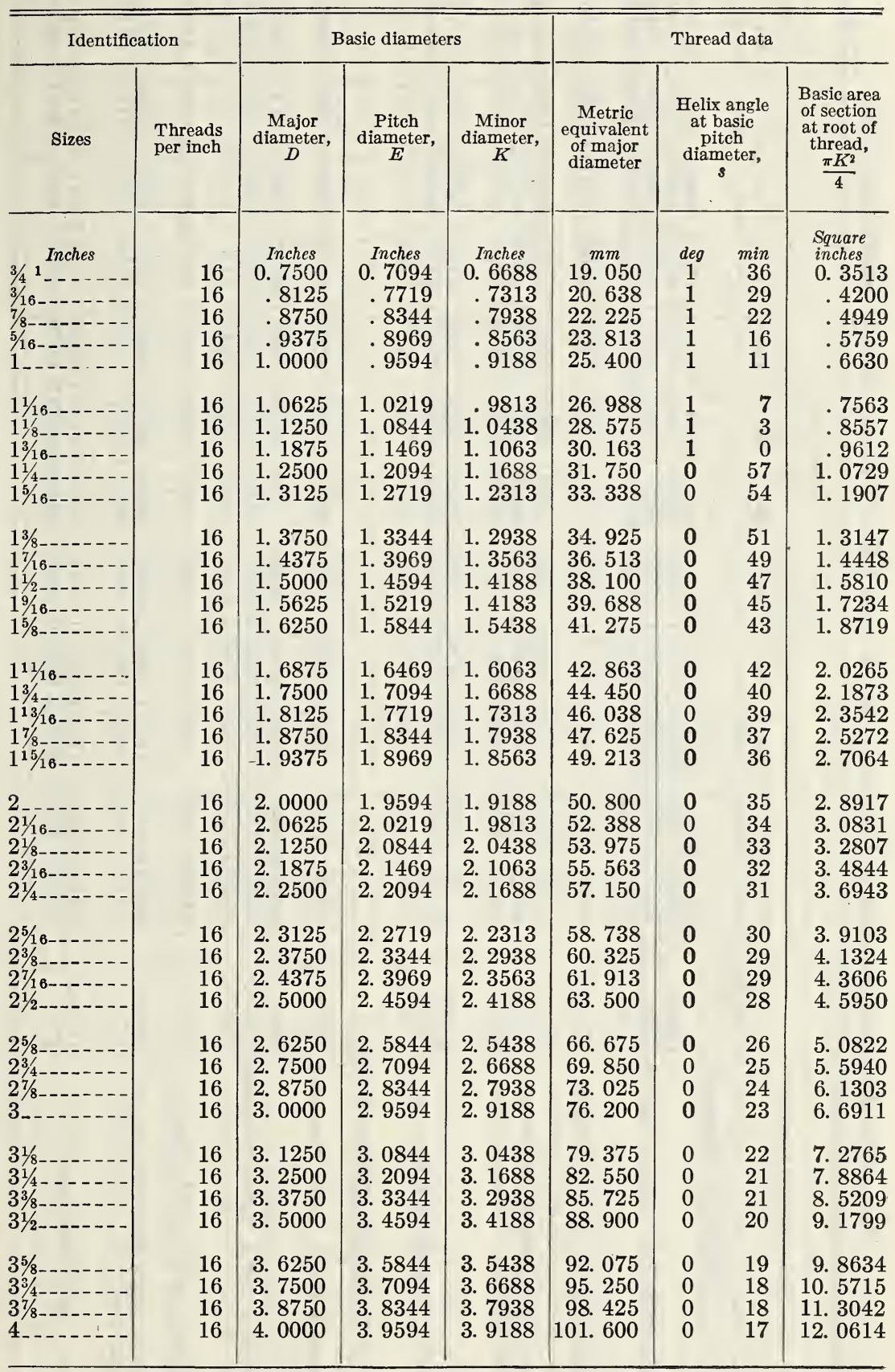

1 Standard size of the American National fine-thread series; 


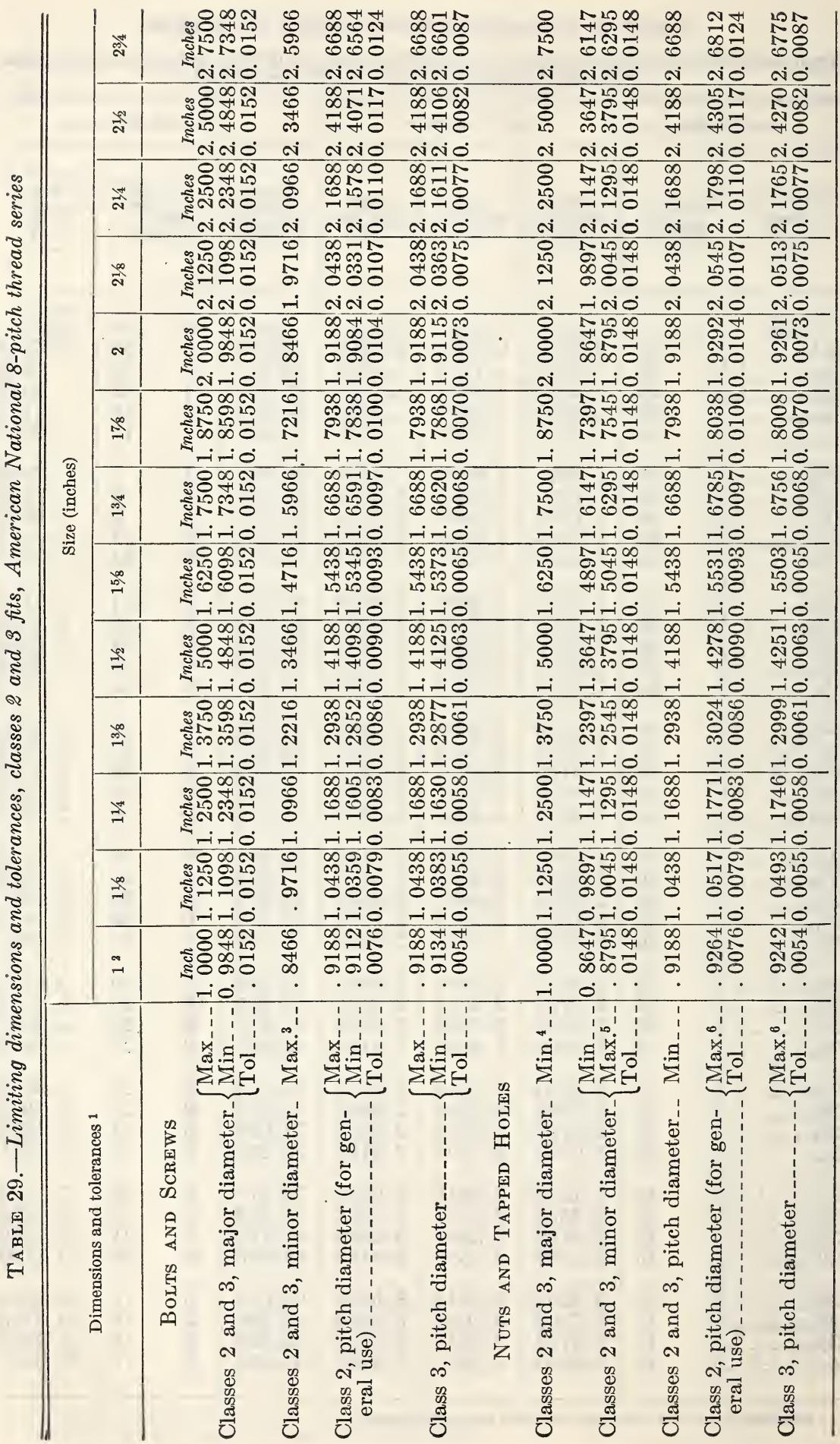




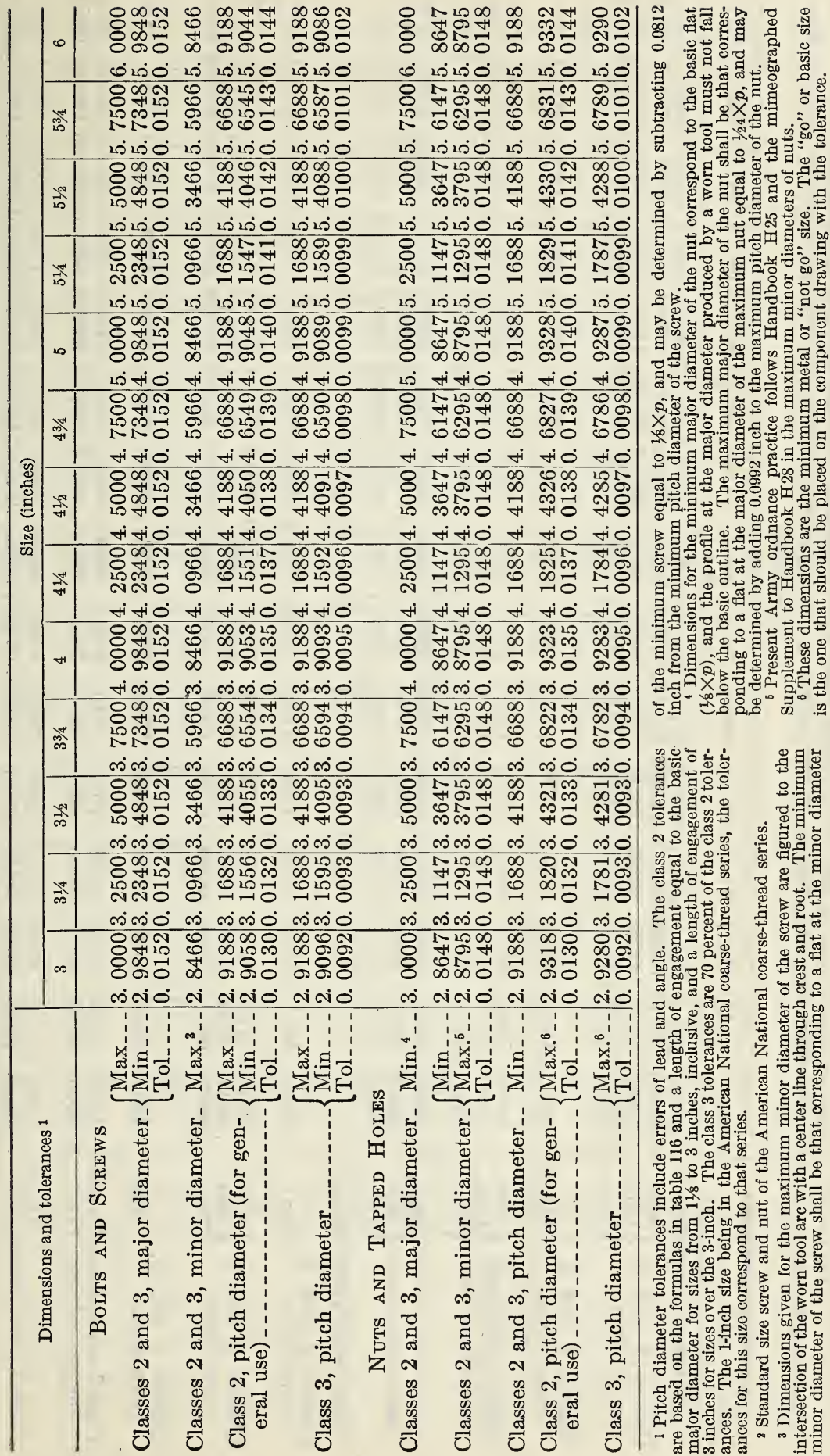




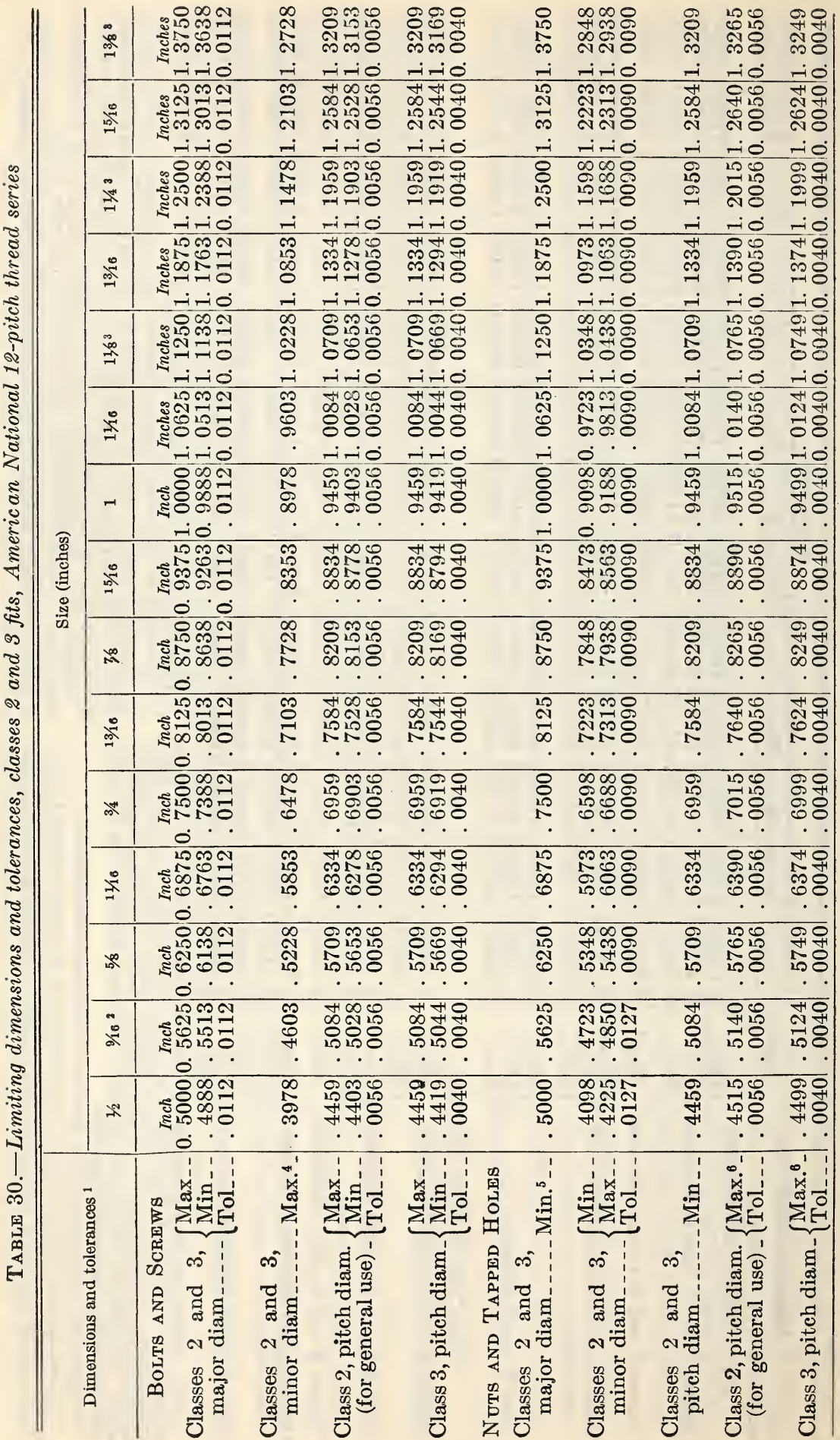




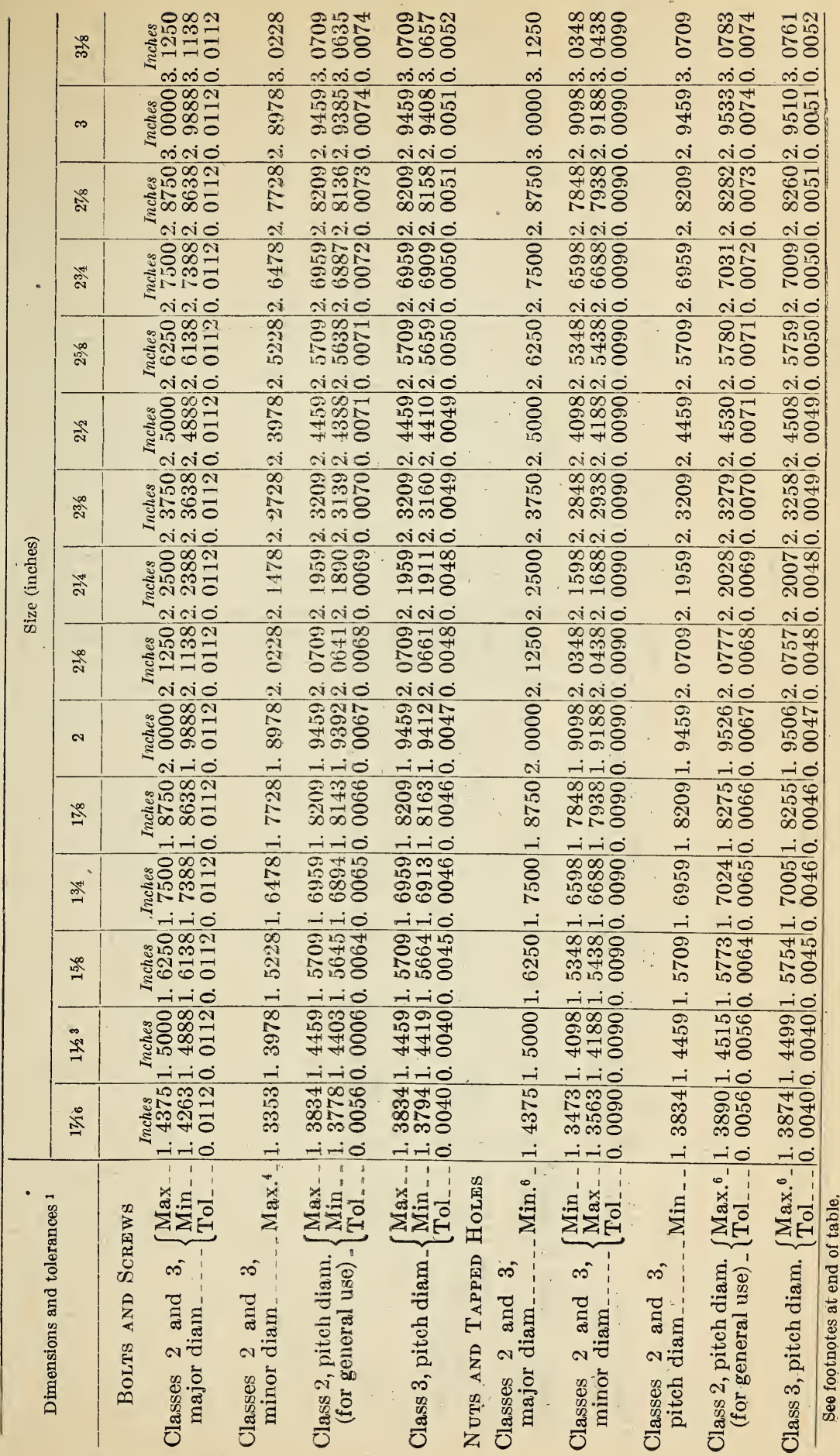

$700485^{\circ}-46-5$ 


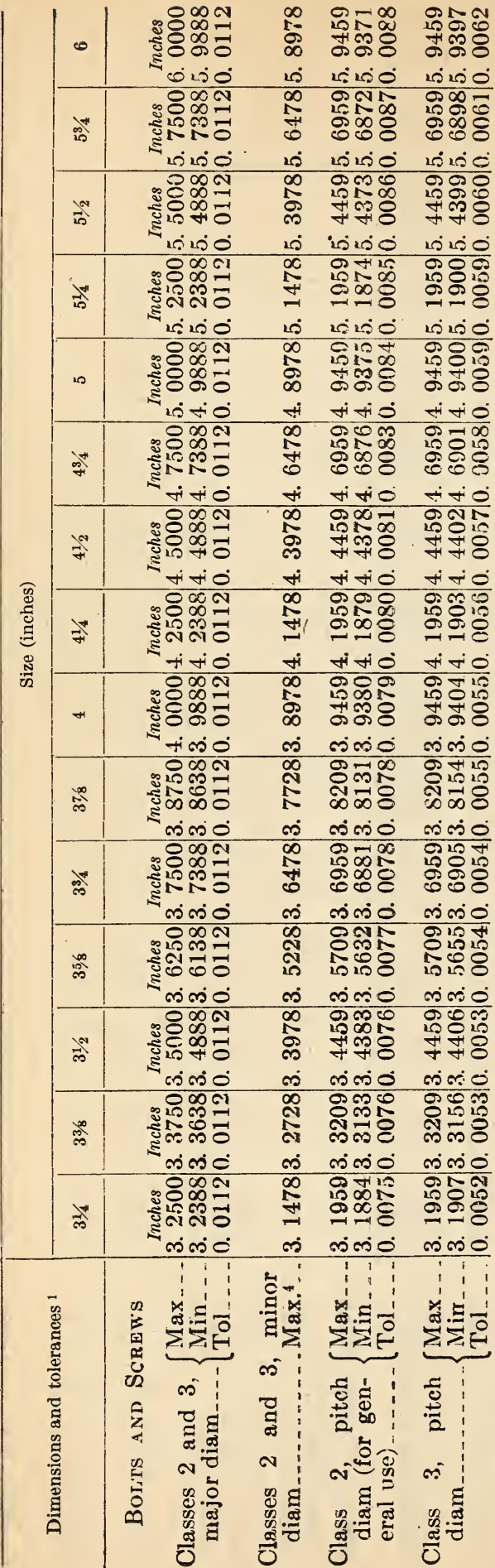




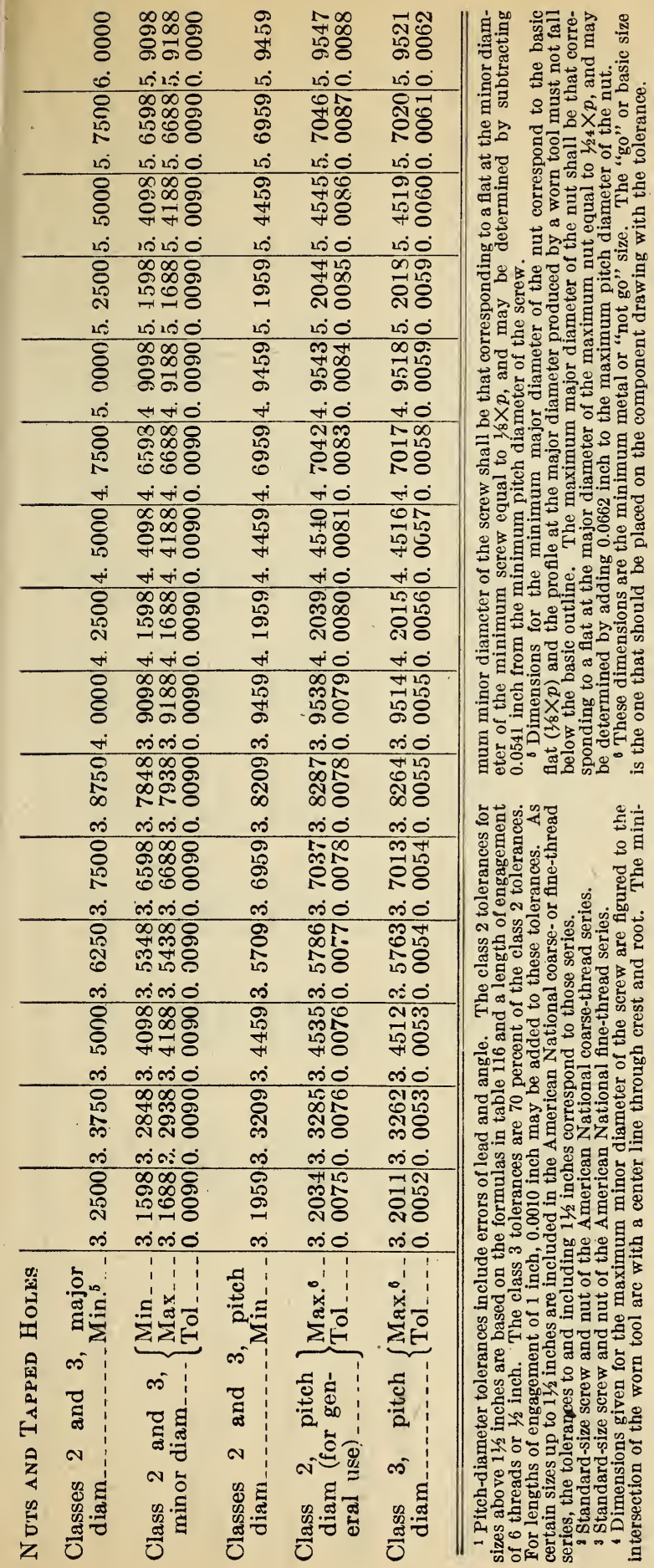




\begin{tabular}{|c|c|c|c|c|c|c|c|c|c|c|}
\hline \multirow{10}{*}{ 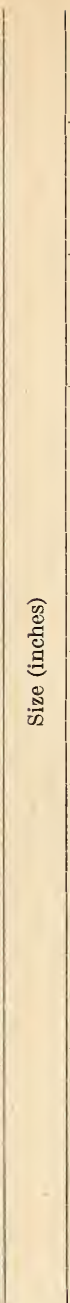 } & 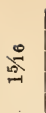 & 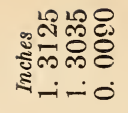 & 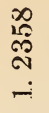 & 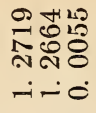 & 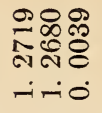 & & $\begin{array}{l}\stackrel{2}{\mathrm{~N}} \\
\stackrel{6}{6} \\
-i\end{array}$ & 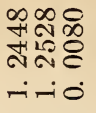 & 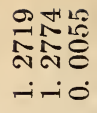 & 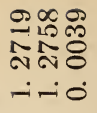 \\
\hline & 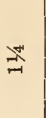 & 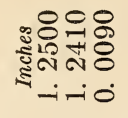 & 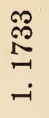 & 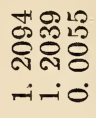 & 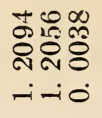 & & $\begin{array}{l}8 \\
\text { \& } \\
\text { on } \\
-1\end{array}$ & 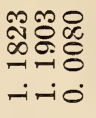 & 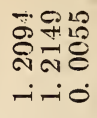 & 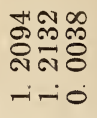 \\
\hline & $\stackrel{\oplus}{=}$ & 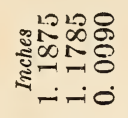 & 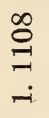 & 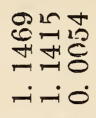 & 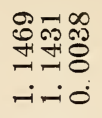 & & 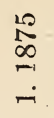 & 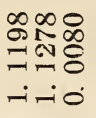 & 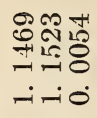 & 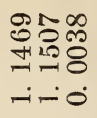 \\
\hline & $\stackrel{\infty}{=}$ & 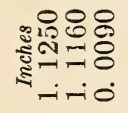 & $\begin{array}{l}\infty \\
\infty \\
0 \\
0 \\
-i\end{array}$ & 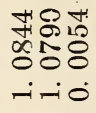 & 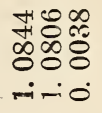 & & 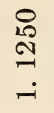 & 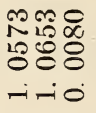 & 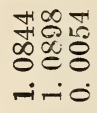 & 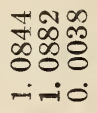 \\
\hline & $\stackrel{0}{\Rightarrow}$ & 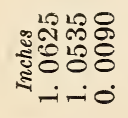 & $\begin{array}{l}\infty \\
10 \\
\infty \\
0\end{array}$ & 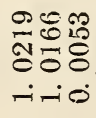 & 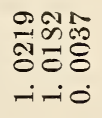 & & $\begin{array}{l}\stackrel{10}{0} \\
\stackrel{8}{0} \\
-1\end{array}$ & 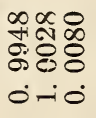 & 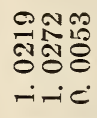 & 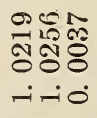 \\
\hline & -1 & 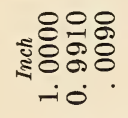 & 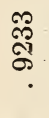 & 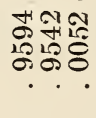 & 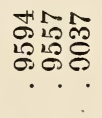 & & $\begin{array}{l}8 \\
8 \\
8 \\
-1\end{array}$ & 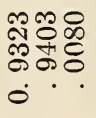 & 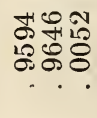 & 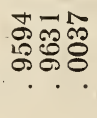 \\
\hline & 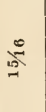 & 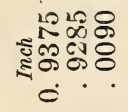 & $\begin{array}{l}\infty \\
\stackrel{8}{0} \\
\infty\end{array}$ & 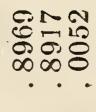 & 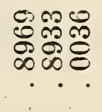 & & $\underset{\mathscr{N}}{\stackrel{1}{2}}$ & $\begin{array}{l}\infty \propto 0 \\
011 \\
0 \stackrel{1}{1} \\
\infty \infty\end{array}$ & 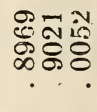 & 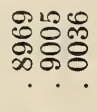 \\
\hline & $\stackrel{\infty}{\infty}$ & 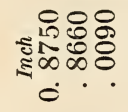 & $\begin{array}{l}\mathscr{\infty} \\
\stackrel{\infty}{R}\end{array}$ & 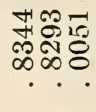 & 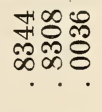 & & $\underset{\infty}{\stackrel{0}{\infty}}$ & 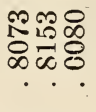 & 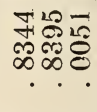 & 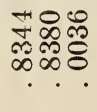 \\
\hline & $\stackrel{0}{\Rightarrow}$ & 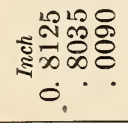 & 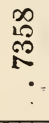 & 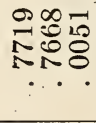 & 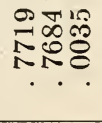 & & $\stackrel{20}{\infty}$ & 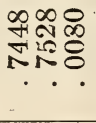 & 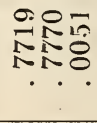 & 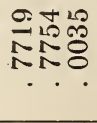 \\
\hline & ळు & 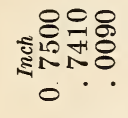 & $\stackrel{\Re}{\stackrel{\oplus}{0}}$ & 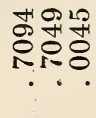 & 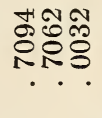 & & 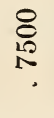 & 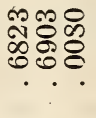 & 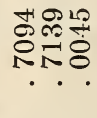 & 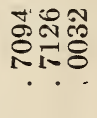 \\
\hline \multicolumn{2}{|c|}{ 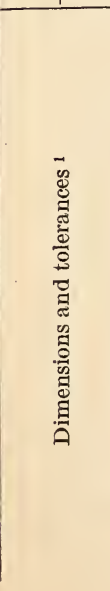 } & 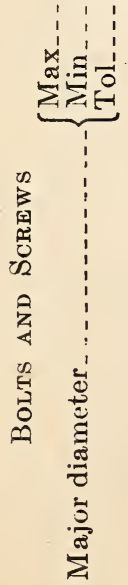 & 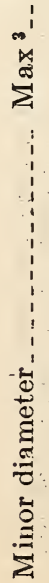 & 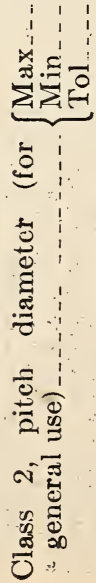 & 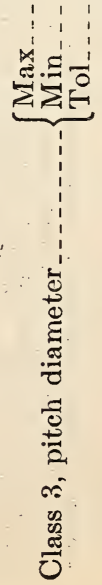 & 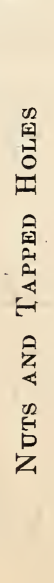 & 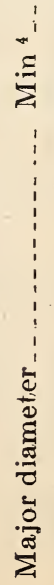 & 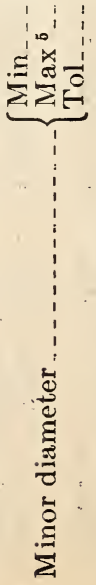 & 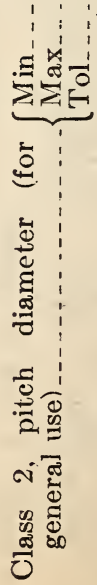 & 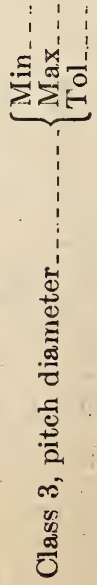 \\
\hline
\end{tabular}




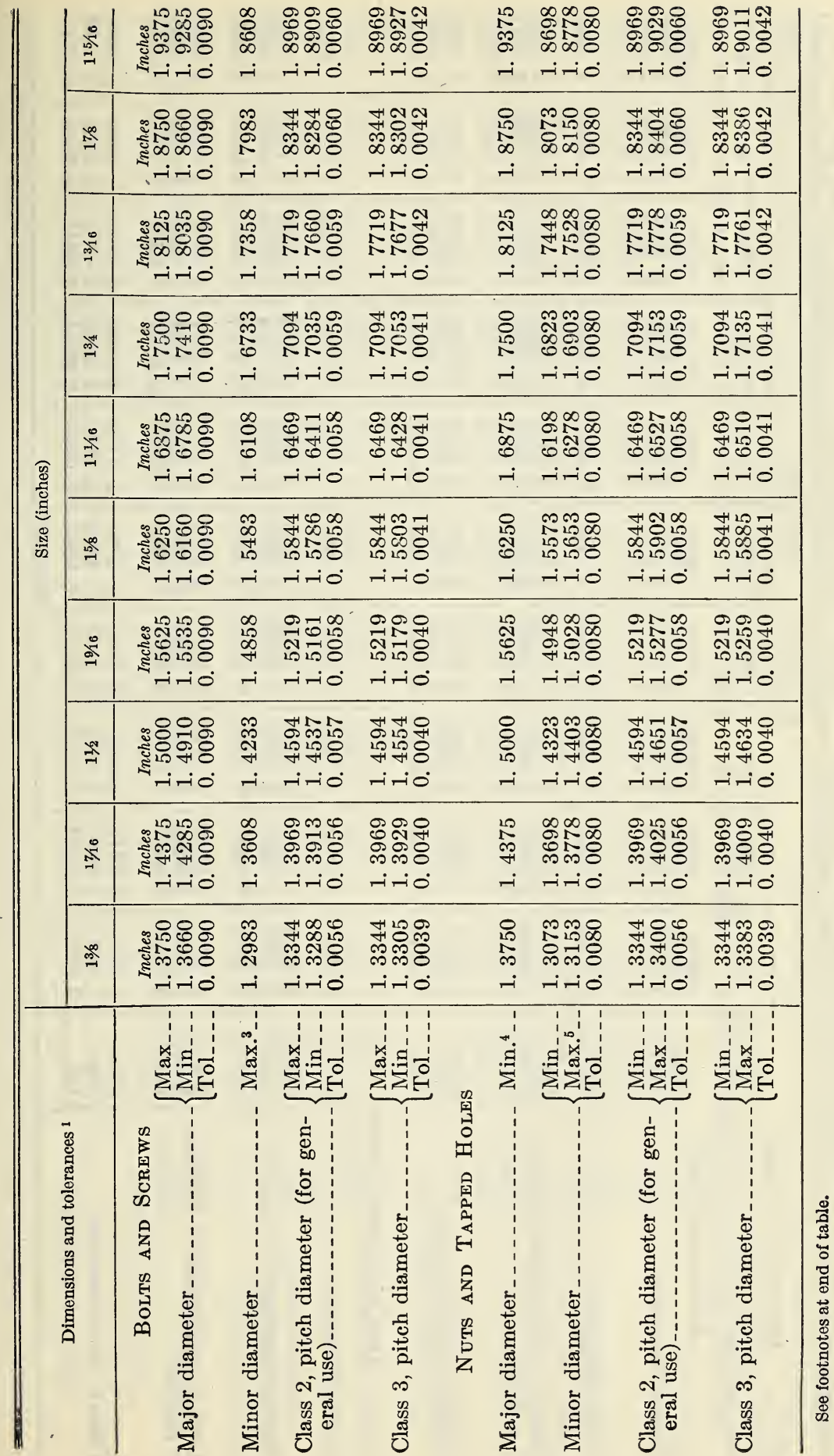




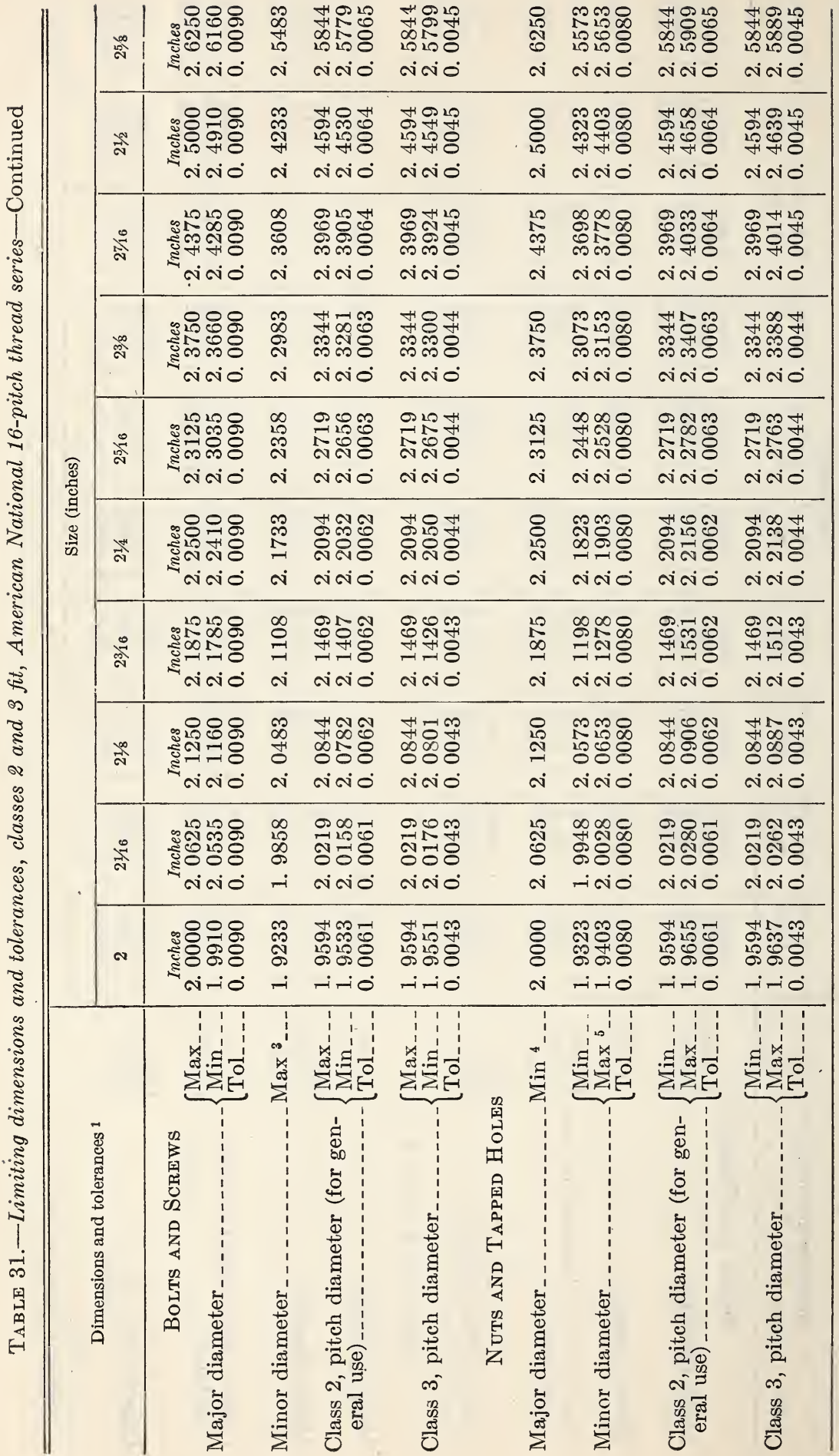




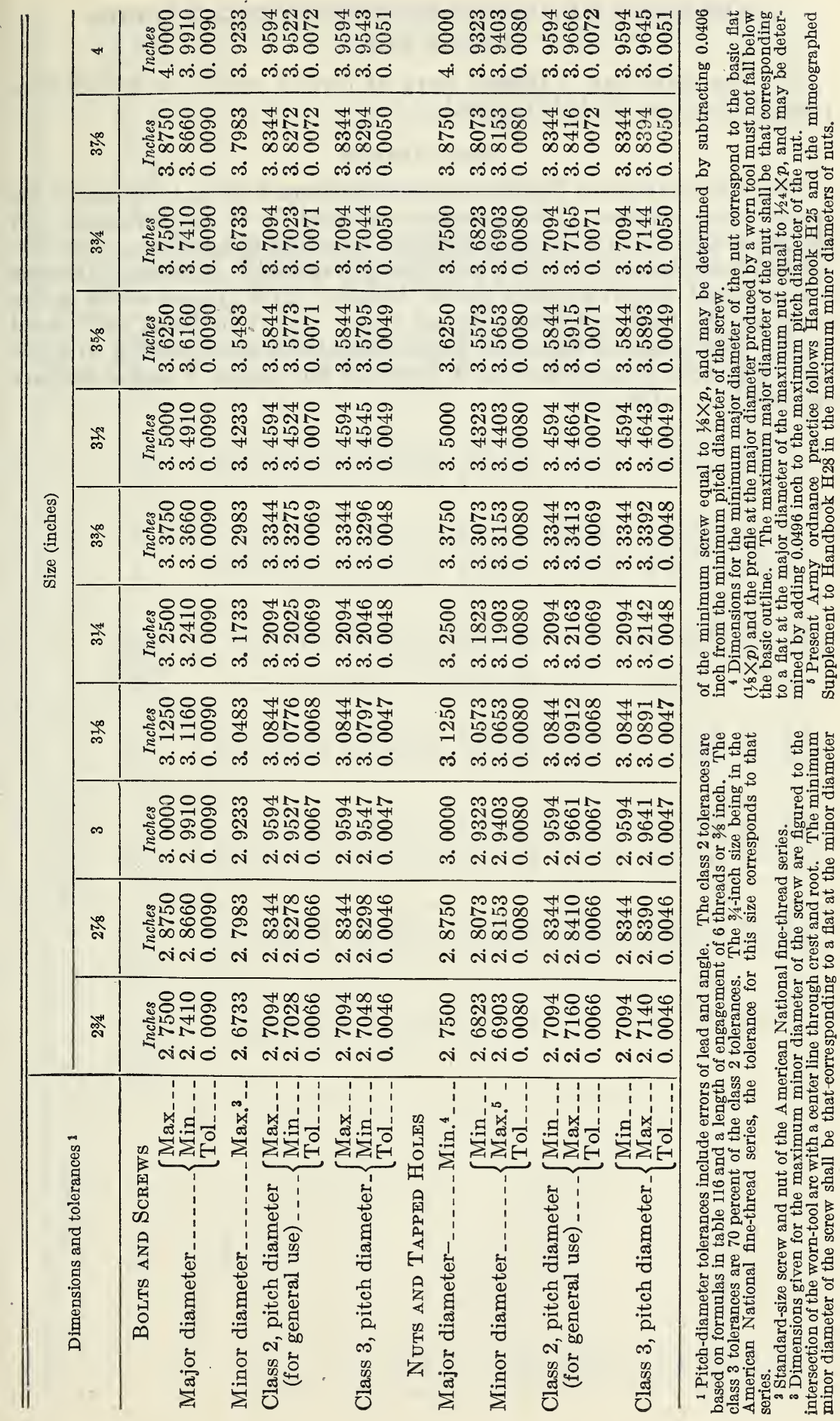




\section{AMERICAN NATIONAL EXTRA-FINE-THREAD SERIES}

\section{FORM OF THREAD}

10. The American National form of thread profile as specified in paragraphs 7 to $7 \mathrm{f}$ shall be used.

\section{THREAD SERIES}

10a. The American National extra-fine-thread series is intended for special uses where (1) thin-walled material is to be threaded, (2) thread depth of nuts clearing ferrules, coupling flanges, etc., must be held to a minimum, and (3) a maximum practicable number of threads are required within a given thread length. This thread series is the same as the SAE extra-fine-thread series, but it includes additional sizes. The nominal sizes and basic dimensions are specified in table 34. Limiting dimensions and tolerances for classes 2 and 3 fits are specified in table 35 . 


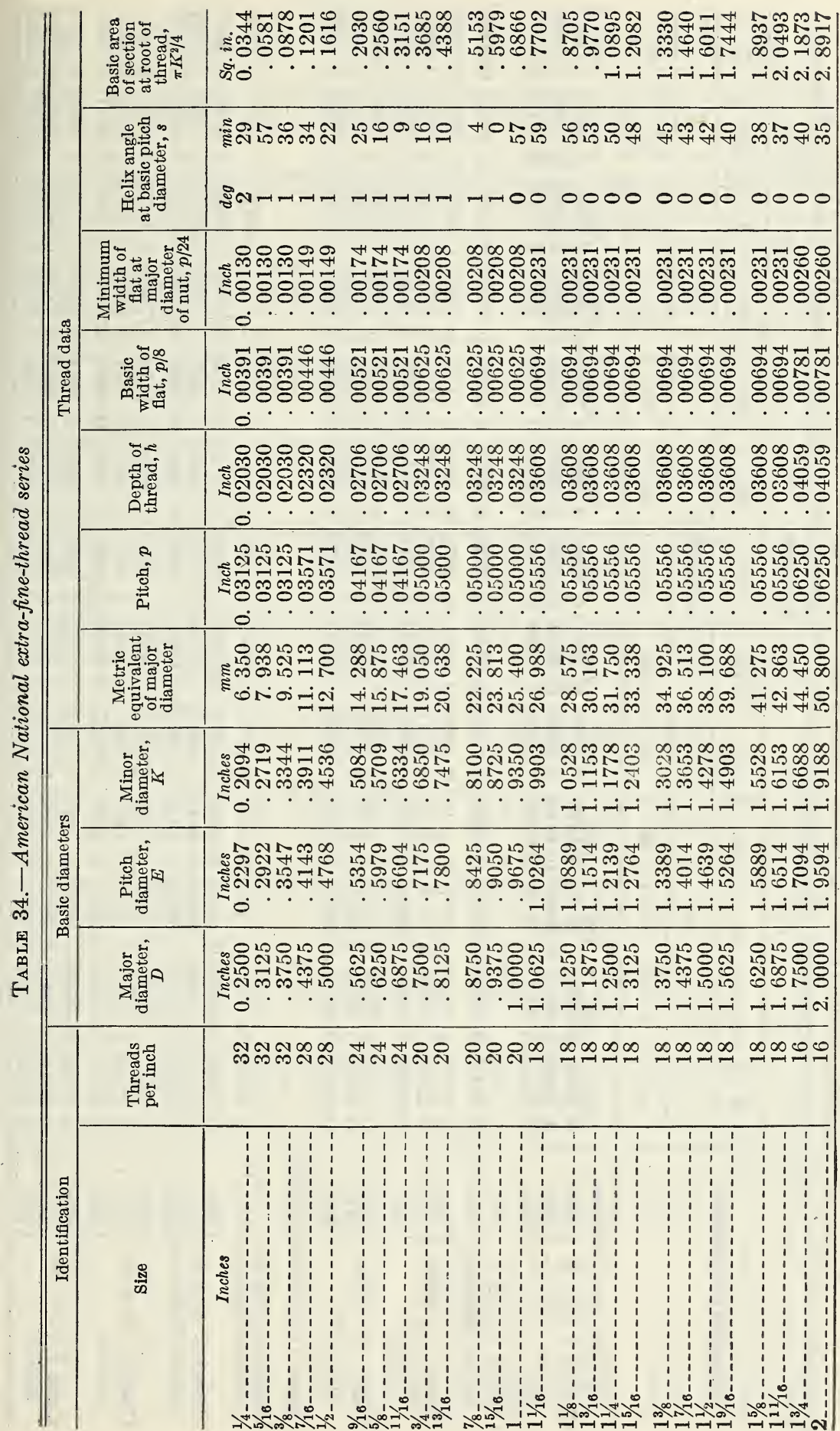




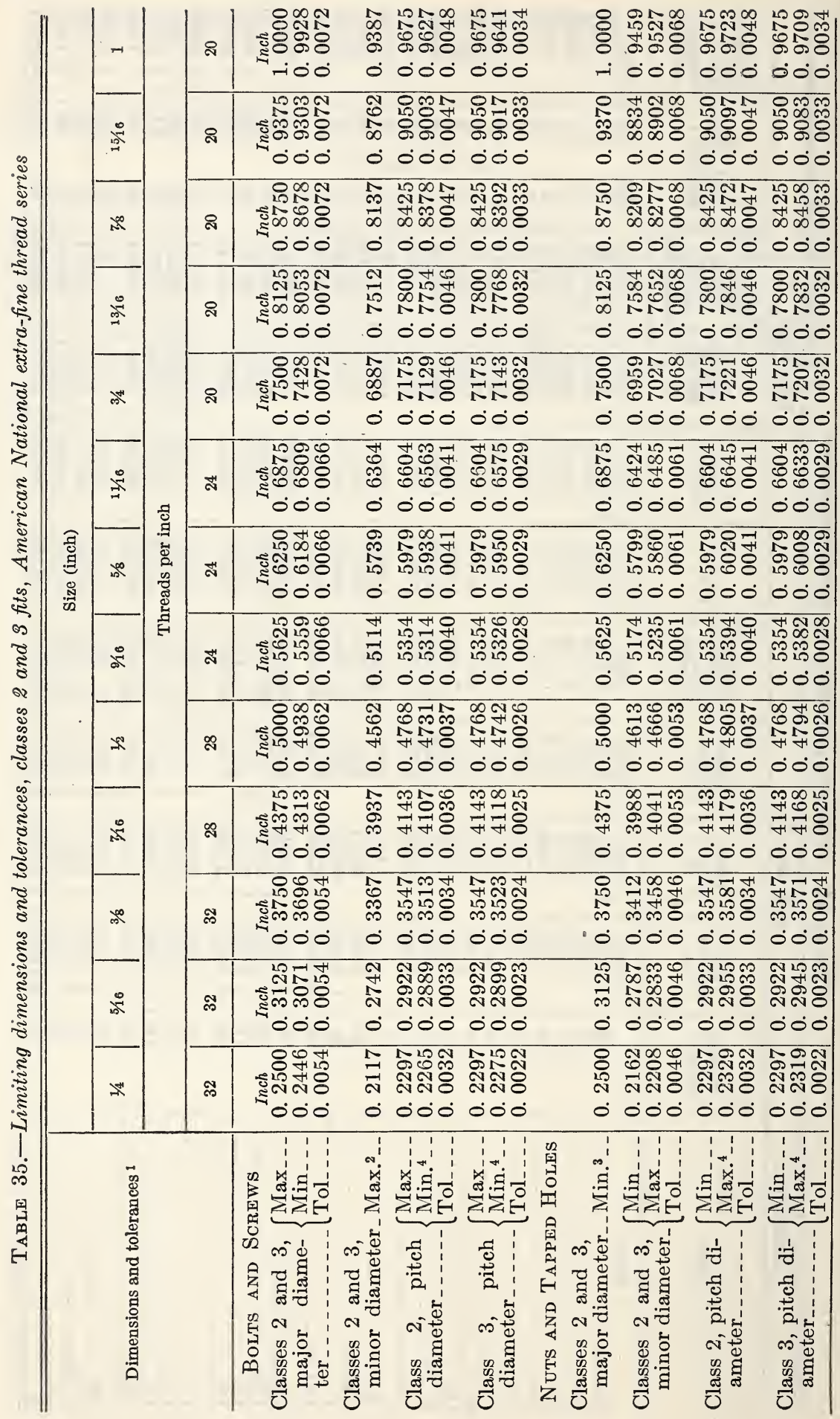




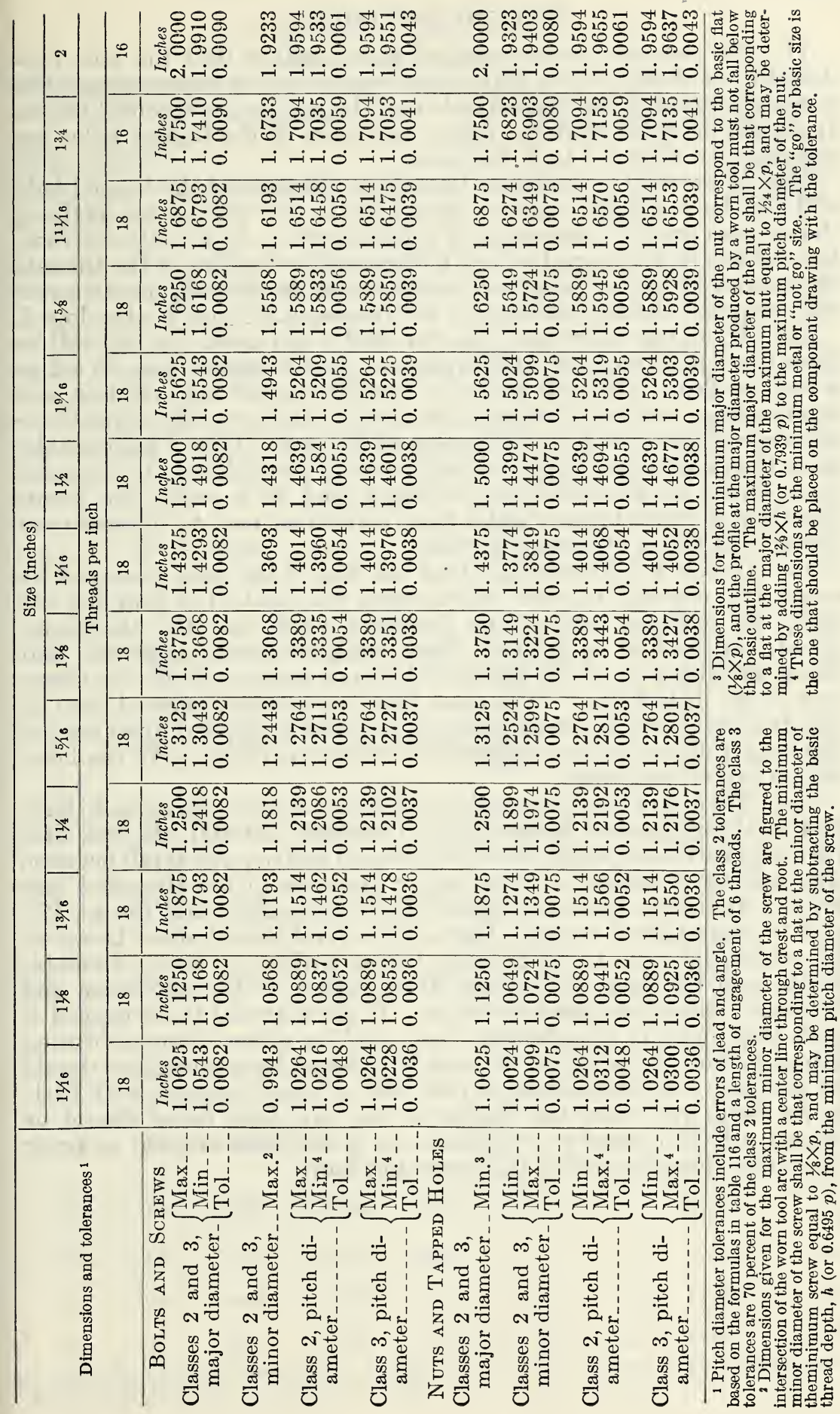




\section{SIZES OF TAP DRILLS}

11. The essential requirement of a tap drill is that the hole produced by it shall be such that, when tapped with a screw thread, the minor diameter of the tapped hole shall be within the specified limits. It should be noted that the minor diameters of the tapped holes are the same for classes 1 to 4 , inclusive.

11a. If the drill is too large, the minor diameter of the tapped hole will also be too large, and the thread in the nut will be too shallow, that is, too small a percentage of a full thread. As an extreme case, the threads in the tapped hole will engage only the tops of the threads on a screw of correct size, and under stress the threads of the screw will strip and the full strength of the fastening will not be developed.

$11 \mathrm{~b}$. If, on the other hand, the tap drill is too small, the tap will be forced to cut a thread of full depth, and in the extreme case to act as a reamer also. This will result in excessive power consumption and tap breakage, and will also make the minor diameter of the tapped hole dependent upon the minor diameter of the tap. This is undesirable, since the minor diameter of the tap is not, in general, held to the same close limits as the other tap elements, and as a result the minor diameter of a hole tapped under these conditions may be in error even though the tap is otherwise correct.

11c. It is a well-known fact that the size of the hole produced by a tap drill depends to some extent upon the method of grinding the drill, the material drilled, the lubricant used, and the alinement, speed, and feed of operation. This being true, it is apparent that fixing the diameter of the tap drill does not completely fix the diameter of the drilled hole. The most that can be accomplished is to fix the drill diameters between certain limits and to depend upon correct grinding, lubrication, and operation to keep the diameter of the holes within prescribed limits.

11d. There are listed in tables 122 and 123 from Handbook H28, and in the additional tables 123 (A) through 123 (D), all drill sizes regularly carried in stock, both English and metric, which fall between the limiting dimensions of the minor diameter of the threaded hole for the American National coarse-, fine-, extra-fine-, and the 8-, 12-, and 16-pitch-thread series. There are several thread sizes, however, for which there are no stock drills falling within the minor diameter limits, and for these the nearest drills outside of the maximum and minimum limits are listed in italics. If the material to be tapped is such that there is considerable "spin-up" on minor diameter during tapping, then the larger of the two drills listed for a given size should be selected. If the material is cast iron or other material with little or no "spin-up", then the smaller of the two drills listed should be chosen. It will usually cut oversize by a sufficient amount to bring the minor diameter above the minimum limit. 
TABLE 122.-Sizes of tap drills

[American National coarse-thread series]

\begin{tabular}{|c|c|c|c|c|c|c|c|}
\hline \multirow{2}{*}{$\begin{array}{l}\text { Size of } \\
\text { thread }\end{array}$} & \multirow{2}{*}{$\begin{array}{l}\text { Threads } \\
\text { per inch }\end{array}$} & \multicolumn{3}{|c|}{ Minor diameter of nut } & \multicolumn{3}{|c|}{$\begin{array}{l}\text { Stock drills corresponding to } 100 \text { percent to } 50 \\
\text { percent of basic thread depth }\end{array}$} \\
\hline & & Basic & Maximum 1 & Minimum & Nominal size & Diameter & $\begin{array}{l}\text { Percentage } \\
\text { of depth of } \\
\text { basic thread }\end{array}$ \\
\hline 1 & 64 & $\begin{array}{c}\text { Inch } \\
0.0527\end{array}$ & $\begin{array}{c}\text { Inch } \\
0.0623\end{array}$ & $\begin{array}{c}\text { Inch } \\
0.0561\end{array}$ & $\left\{\begin{array}{l}1.45 \mathrm{~mm} \ldots \\
1.50 \mathrm{~mm} \\
1.55 \mathrm{~mm}\end{array}\right.$ & $\begin{array}{l}\text { Inch } \\
0.0571 \\
.0591 \\
.0610\end{array}$ & $\begin{array}{l}78 \\
68 \\
59\end{array}$ \\
\hline$z$ & 56 & .0628 & .0737 & .0667 & $\left\{\begin{array}{l}\# 51 \ldots \ldots \\
\# 50 \ldots \ldots \\
\# 49\end{array}\right.$ & $\begin{array}{l}.0670 \\
.0700 \\
.0730\end{array}$ & $\begin{array}{l}82 \\
69 \\
56\end{array}$ \\
\hline 3 & 48 & .0719 & .0841 & .0764 & $\left\{\begin{array}{l}5 / 64 \text { in } \\
\# 446 \\
2.10 \mathrm{~mm}\end{array}\right.$ & $\begin{array}{l}.0781 \\
.0810 \\
.0827\end{array}$ & $\begin{array}{l}77 \\
67 \\
60\end{array}$ \\
\hline 4 & 40 & .0795 & .0938 & .0849 & $\left\{\begin{array}{l}\# 44 \\
\# 43 \\
2.30 \mathrm{~mm} \\
3 / 32 \text { in }\end{array}\right.$ & $\begin{array}{l}.0860 \\
.0890 \\
.0906 \\
.0937\end{array}$ & $\begin{array}{l}80 \\
71 \\
66 \\
56\end{array}$ \\
\hline 5 & 40 & .0925 & .1062 & .0979 & $\left\{\begin{array}{l}\# 39 \\
\# 38^{3} \\
2.60 \mathrm{~mm} \\
\# 37\end{array}\right.$ & $\begin{array}{l}.0995 \\
.1015 \\
.1024 \\
.1040\end{array}$ & $\begin{array}{l}79 \\
72 \\
70 \\
65\end{array}$ \\
\hline 6 & 32 & .0974 & .1145 & .1042 & $\left\{\begin{array}{l}\# 36 \ldots \ldots \ldots \\
764 \text { in } \\
\# 33\end{array}\right.$ & $\begin{array}{l}.1065 \\
.1094 \\
.1130\end{array}$ & $\begin{array}{l}78 \\
70 \\
62\end{array}$ \\
\hline 8 & 32 & .1234 & 1384 & .1302 & $\left\{\begin{array}{l}3.40 \mathrm{~mm} \\
\# 29 \\
3.50 \mathrm{~mm}\end{array}\right.$ & $\begin{array}{l}.1339 \\
.1360 \\
.1378\end{array}$ & $\begin{array}{l}74 \\
69 \\
65\end{array}$ \\
\hline 10 & 24 & .1359 & .1559 & 1449 & $\left\{\begin{array}{l}\# 26 \ldots \ldots \\
\# 24 \ldots \ldots\end{array}\right.$ & $\begin{array}{l}.1470 \\
.1520\end{array}$ & $\begin{array}{l}79 \\
70\end{array}$ \\
\hline 12 & 24 & .1619 & .1801 & .1709 & $\left\{\begin{array}{l}11 / 64 \text { in } \ldots \ldots \\
\# 17 \\
\# 16 \\
\# 15\end{array}\right.$ & $\begin{array}{l}.1719 \\
.1730 \\
.1770 \\
.1800\end{array}$ & $\begin{array}{l}82 \\
79 \\
72 \\
67\end{array}$ \\
\hline $1 / 4$ & 20 & .1850 & .2060 & .1959 & $\left\{\begin{array}{l}\# 9 \\
\# 8 \\
13 \% 64 \text { in }\end{array}\right.$ & $\begin{array}{l}.1960 \\
.1990 \\
.2031\end{array}$ & $\begin{array}{l}83 \\
79 \\
72\end{array}$ \\
\hline $5 / 16$ & 18 & .2403 & .2630 & .2524 & $\left\{\begin{array}{l}F_{-} \ldots \ldots \\
G_{\ldots}\end{array}\right.$ & $\begin{array}{l}.2570 \\
.2610\end{array}$ & $\begin{array}{l}77 \\
71\end{array}$ \\
\hline $3 / 8$ & 16 & .2938 & .3184 & .3073 & $\left\{\begin{array}{l}5 / 16 \text { in } \ldots \\
O\end{array}\right.$ & $\begin{array}{l}.3125 \\
.3160\end{array}$ & $\begin{array}{l}77 \\
73\end{array}$ \\
\hline $7 / 16$ & 14 & .3447 & .3721 & .3602 & $U_{\ldots} \ldots \ldots$ & .3680 & 75 \\
\hline $1 / 2$ & 13 & .4001 & .4290 & .4167 & $27 / 64$ in $\ldots$ & .4219 & 78 \\
\hline$\% / 10$ & 12 & .4542 & .4850 & .4723 & $31 / 64$ in $\ldots$ & .4844 & 72 \\
\hline
\end{tabular}

1 Present Army Ordnance practice follows Handbook H25 and the mimeographed Supplement to Handbook $\mathrm{H} 28$ in the maximum minor diameters of nuts.

8 See footnote at end of table 123 (D). 
TABLE 122.-Sizes of tap drills-Continued

[American National coarse-thread series]

\begin{tabular}{|c|c|c|c|c|c|c|c|}
\hline \multirow{2}{*}{$\begin{array}{l}\text { Size of } \\
\text { thread }\end{array}$} & \multirow{2}{*}{$\begin{array}{l}\text { Threads } \\
\text { per inch }\end{array}$} & \multicolumn{3}{|c|}{ Minor diameter of nut } & \multicolumn{3}{|c|}{$\begin{array}{l}\text { Stock drills corresponding to } 100 \text { percent to } 50 \\
\text { percent of basic thread depth }\end{array}$} \\
\hline & & Basic & Maximum & Minimum & Nominal size & Diameter & $\begin{array}{l}\text { Percentage } \\
\text { of depth of } \\
\text { basic thread }\end{array}$ \\
\hline $5 / 8$ & 11 & $\begin{array}{l}\text { Inch } \\
.5069\end{array}$ & $\begin{array}{l}\text { Inch } \\
.5397\end{array}$ & $\begin{array}{l}\text { Inch } \\
.5266\end{array}$ & $\left\{\begin{array}{l}17 / 3{ }_{2} \text { in } \\
13.5 \mathrm{~mm}\end{array}\right.$ & $\begin{array}{l}\text { Inch } \\
.5312 \\
.5315\end{array}$ & $\begin{array}{l}79 \\
79\end{array}$ \\
\hline $3 / 4$ & 10 & .6201 & .6553 & .6417 & $16.5 \mathrm{~mm}$ & .6496 & 77 \\
\hline $7 / 8$ & 9 & .7307 & .7689 & .7547 & $\left\{\begin{array}{l}49 / 64 \text { in } \\
19.5 \mathrm{~mm}\end{array}\right.$ & $\begin{array}{l}.7656 \\
.7677\end{array}$ & $\begin{array}{l}76 \\
74\end{array}$ \\
\hline 1 & 8 & .8376 & .8795 & .8647 & $\left\{\begin{array}{l}22 \mathrm{~mm}_{-} \\
7 / 8 \mathrm{in}\end{array}\right.$ & $\begin{array}{l}.8661 \\
.8750\end{array}$ & $\begin{array}{l}82 \\
77\end{array}$ \\
\hline $11 / 8$ & 7 & .9394 & .9858 & .9704 & $\left\{\begin{array}{l}25 \mathrm{~mm} \\
636^{\prime} \mathrm{in}_{-}\end{array}\right.$ & $\begin{array}{l}.9842 \\
.9844\end{array}$ & $\begin{array}{l}76 \\
76\end{array}$ \\
\hline $11 / 4$ & 7 & 1. 0644 & 1. 1108 & 1. 0954 & $\left\{\begin{array}{l}28 \mathrm{~mm}_{-} \\
17 / 64 \mathrm{in}_{-}\end{array}\right.$ & $\begin{array}{l}\text { 1. } 1024 \\
\text { 1. } 1094\end{array}$ & $\begin{array}{l}80 \\
76\end{array}$ \\
\hline $13 / 8$ & 6 & 1. 1585 & 1. 2126 & 1. 1946 & $\left\{\begin{array}{l}30.5 \mathrm{~mm} \\
113 / 64 \mathrm{in} .\end{array}\right.$ & $\begin{array}{l}\text { 1. } 2008 \\
\text { 1. } 2031\end{array}$ & $\begin{array}{l}80 \\
79\end{array}$ \\
\hline $11 / 2$ & 6 & 1. 2835 & 1. 3376 & 1. 3196 & $121 / 64$ in. & 1. 3281 & 79 \\
\hline $13 / 4$ & 5 & 1. 4902 & 1. 5551 & 1. 5335 & $\left\{\begin{array}{l}39 \mathrm{~mm}^{35 / 64} \mathrm{in}_{1} \\
39.5 \mathrm{~mm}_{-}\end{array}\right.$ & $\begin{array}{l}\text { 1. } 5354 \\
\text { 1. } 5469 \\
\text { 1. } 5551\end{array}$ & $\begin{array}{l}83 \\
78 \\
75\end{array}$ \\
\hline 2 & $41 / 2$ & 1. 7113 & 1. 7835 & 1. 7594 & $\left\{\begin{array}{l}14 \% 6 \mathrm{in}^{4} \\
45 \mathrm{~mm} \\
125 / 32 \mathrm{in}\end{array}\right.$ & $\begin{array}{l}\text { 1. } 7656 \\
\text { 1. } 7716 \\
\text { 1. } 7812\end{array}$ & $\begin{array}{l}81 \\
79 \\
76\end{array}$ \\
\hline $21 / 4$ & $41 \frac{1}{2}$ & 1. 9613 & 2. 0335 & 2. 0094 & $\left\{\begin{array}{l}21 / 64 \text { in } \\
51.5 \mathrm{~mm}^{1 / 32} \text { in } \\
2 .\end{array}\right.$ & $\begin{array}{l}\text { 2. } 0156 \\
\text { 2. } 0276 \\
\text { 2. } 0312\end{array}$ & $\begin{array}{l}81 \\
77 \\
76\end{array}$ \\
\hline $21 / 2$ & 4 & 2. 1752 & 2. 2564 & 2. 2294 & $\left\{\begin{array}{l}2^{15} / 64 \mathrm{in} \\
57 \mathrm{~mm} \\
21 / 4 \mathrm{in}\end{array}\right.$ & $\begin{array}{l}\text { 2. } 2344 \\
\text { 2. } 2441 \\
\text { 2. } 2500\end{array}$ & $\begin{array}{l}82 \\
79 \\
77\end{array}$ \\
\hline $23 / 4$ & 4 & 2. 4252 & 2. 5064 & 2. 4794 & $\left\{\begin{array}{l}63 \mathrm{~mm} \\
2^{31} 1 / 64 \mathrm{in}_{-} \\
63.5 \mathrm{~mm} \\
21 / 2 \mathrm{in} .\end{array}\right.$ & $\begin{array}{l}\text { 2. } 4803 \\
\text { 2. } 4844 \\
\text { 2. } 5000 \\
\text { 2. } 5000\end{array}$ & $\begin{array}{l}83 \\
82 \\
77 \\
77\end{array}$ \\
\hline 3 & 4 & 2. 6752 & 2. 7564 & 2. 7294 & $\left\{\begin{array}{l}247 / 64 \mathrm{in} \\
69.5 \mathrm{~mm} \\
23 / 4 \mathrm{in} \\
70 \mathrm{~mm}\end{array}\right.$ & $\begin{array}{l}\text { 2. } 7344 \\
\text { 2. } 7362 \\
\text { 2. } 7500 \\
\text { 2. } 7559\end{array}$ & $\begin{array}{l}82 \\
81 \\
77 \\
75\end{array}$ \\
\hline $31 / 4$ & 4 & 2. 9252 & 3. 0064 & 2. 9794 & $\left\{\begin{array}{l}2^{63} / 64 \text { in } \\
76 \mathrm{~mm} \\
3\end{array}\right.$ & $\begin{array}{l}\text { 2. } 9844 \\
\text { 2. } 9921 \\
\text { 3. } 0000\end{array}$ & $\begin{array}{l}82 \\
79 \\
77\end{array}$ \\
\hline $31 / 2$ & 4 & 3. 1752 & 3. 2564 & 3. 2294 & $31 / 4$ in & 3. 2500 & 77 \\
\hline $33 / 4$ & 4 & 3. 4252 & 3. 5064 & 3. 4794 & $31 / 2$ in $\ldots$ & 3. 5000 & 77 \\
\hline
\end{tabular}


TABLE 123.-Sizes of tap drills

[American National fine-thread series] ${ }^{1}$

\begin{tabular}{|c|c|c|c|c|c|c|c|}
\hline \multirow{2}{*}{$\begin{array}{l}\text { Size of } \\
\text { thread }\end{array}$} & \multirow{2}{*}{$\begin{array}{l}\text { Threads } \\
\text { per inch }\end{array}$} & \multicolumn{3}{|c|}{ Minor diameter of nut } & \multicolumn{3}{|c|}{$\begin{array}{l}\text { Stock drills corresponding to } 100 \text { percent to } 50 \\
\text { percent of basic thread depth }\end{array}$} \\
\hline & & Basic & Maximum ${ }^{2}$ & Minimum & Nominal size & Diameter & $\begin{array}{l}\text { Percentage } \\
\text { of depth of } \\
\text { basic thread }\end{array}$ \\
\hline $0_{--}$ & 80 & $\begin{array}{c}\text { Inch } \\
0.0438\end{array}$ & $\begin{array}{c}\text { Inch } \\
0.0514\end{array}$ & $\begin{array}{c}\text { Inch } \\
0.0465\end{array}$ & $\left\{\begin{array}{l}3 / 64 \text { in } \\
1.25 \mathrm{~mm}\end{array}\right.$ & $\begin{array}{l}\text { Inch } \\
0.0469 \\
.0492\end{array}$ & $\begin{array}{l}81 \\
67\end{array}$ \\
\hline 1.- & 72 & .0550 & .0634 & .0580 & $\left\{\begin{array}{l}1.50 \mathrm{~mm}_{-} \ldots \\
1.55 \mathrm{~mm}_{-}\end{array}\right.$ & $\begin{array}{l}.0591 \\
.0610\end{array}$ & $\begin{array}{l}77 \\
67\end{array}$ \\
\hline $2 \ldots$ & 64 & .0657 & .0746 & .0691 & $\left\{\begin{array}{l}\# 50 \ldots \ldots- \\
\# 49 \ldots \ldots\end{array}\right.$ & $\begin{array}{l}.0700 \\
.0730\end{array}$ & $\begin{array}{l}79 \\
64\end{array}$ \\
\hline $3 \ldots$ & 56 & .0758 & .0856 & .0797 & $\left\{\begin{array}{l}\# 46 \\
2.10 \mathrm{~mm} \\
\# 44\end{array}\right.$ & $\begin{array}{l}.0810 \\
.0827 \\
.0860\end{array}$ & $\begin{array}{l}78 \\
70 \\
56\end{array}$ \\
\hline $4--$ & 48 & .0849 & .0960 & .0894 & $\left\{\begin{array}{l}2.30 \mathrm{~mm} \\
3 / 32 \text { in } \\
\# 41\end{array}\right.$ & $\begin{array}{l}.0906 \\
.0937 \\
.0960\end{array}$ & $\begin{array}{l}79 \\
68 \\
59\end{array}$ \\
\hline 5_- & 44 & .0955 & .1068 & .1004 & $\left\{\begin{array}{l}2.60 \mathrm{~mm}-\ldots \\
\# 37 \\
\# 36\end{array}\right.$ & $\begin{array}{l}.1024 \\
.1040 \\
.1065\end{array}$ & $\begin{array}{l}77 \\
71 \\
63\end{array}$ \\
\hline 6.- & 40 & .1055 & .1179 & .1109 & $\left\{\begin{array}{l}\# 33_{2} \ldots \ldots \\
\# 32 \ldots\end{array}\right.$ & $\begin{array}{l}.1130 \\
.1160\end{array}$ & $\begin{array}{l}77 \\
68\end{array}$ \\
\hline 8_- & 36 & .1279 & .1402 & 1339 & $\left\{\begin{array}{l}3.40 \mathrm{~mm} \\
\# 29 \\
3.50 \mathrm{~mm} \\
9 \% 6 \text { in }\end{array}\right.$ & $\begin{array}{l}1339 \\
.1360 \\
.1378 \\
.1406\end{array}$ & $\begin{array}{l}83 \\
78 \\
73 \\
65\end{array}$ \\
\hline $10_{-}$ & 32 & .1494 & .1624 & .1562 & $\left\{\begin{array}{l}5 / 32 \text { in } \\
\# 21^{3} \ldots \ldots \\
\# 20 \\
\# 19\end{array}\right.$ & $\begin{array}{l}1562 \\
.1590 \\
.1610 \\
.1660\end{array}$ & $\begin{array}{l}83 \\
76 \\
71 \\
59\end{array}$ \\
\hline $12_{--}$ & 28 & .1696 & .1835 & .1773 & $\left\{\begin{array}{l}\# 15 \\
4.70 \mathrm{~mm} \# 13 \\
3 / 16 \text { in }\end{array}\right.$ & $\begin{array}{l}.1800 \\
.1850 \\
.1875\end{array}$ & $\begin{array}{l}78 \\
67 \\
61\end{array}$ \\
\hline $1 / 4--$ & 28 & .2036 & .2173 & .2113 & $\# 3 \ldots \ldots \ldots$ & .2130 & 80 \\
\hline $5 / 16 \mathrm{f}$ & 24 & .2584 & .2739 & .2674 & $\left\{\begin{array}{l}17 / 64 \text { in } \\
I\end{array}\right.$ & $\begin{array}{l}.2656 \\
.2720\end{array}$ & $\begin{array}{l}87 \\
75\end{array}$ \\
\hline $3 / 8--$ & 24 & .3209 & .3364 & .3299 & $Q_{-}$ & .3320 & 79 \\
\hline
\end{tabular}

1 Drill sizes up to $1 / 2$ inch are in agreement with ASA B5.12-1940, Twist Drills, Straight Shank, published by the ASME, 29 West 39th Street, New York, N. Y.

2 Present Army Ordnance practice follows NBS Handbook H25 and the mimeographed Supplement to Handbook H28 in the maximum minor diameters of nuts.

3 See footnote at end of table 123 (D). 
TABLE 123.-Sizes of tap drills-Continued

[American National fine-thread series]

\begin{tabular}{|c|c|c|c|c|c|c|c|}
\hline \multirow{2}{*}{$\begin{array}{l}\text { Size of } \\
\text { thread }\end{array}$} & \multirow{2}{*}{$\begin{array}{l}\text { Threads } \\
\text { per inch }\end{array}$} & \multicolumn{3}{|c|}{ Minor diameter of nut } & \multicolumn{3}{|c|}{$\begin{array}{l}\text { Stock drills corresponding to } 100 \text { percent to } 50 \\
\text { percent of basic thread depth }\end{array}$} \\
\hline & & Basic & Maximum & Minimum & Nominal size & Diameter & $\begin{array}{l}\text { Percentage } \\
\text { of depth of } \\
\text { basic thread }\end{array}$ \\
\hline $7 / 16-$ & 20 & $\begin{array}{c}\text { Inch } \\
0.3725\end{array}$ & $\begin{array}{c}\text { Inch } \\
\text { 0. } 3906\end{array}$ & $\begin{array}{c}\text { Inch } \\
0.3834\end{array}$ & $\left\{\begin{array}{l}\mathrm{W}_{25}-- \\
25 / 64\end{array}\right.$ & $\begin{array}{c}\text { Inch } \\
\text { 0. } 3860 \\
.3906\end{array}$ & $\begin{array}{l}79 \\
72\end{array}$ \\
\hline $1 / 2--$ & 20 & .4350 & .4531 & .4459 & $29 / 64$ in $\ldots$. & .4531 & 72 \\
\hline $9 / 16--$ & 18 & . 4903 & .5100 & .5024 & $0.5062 \ldots$ & .5062 & 78 \\
\hline $5 / 8--$ & 18 & .5528 & .5725 & .5649 & $14.5 \mathrm{~mm}_{\ldots} \ldots$ & .5709 & 75 \\
\hline $3 / 4-$ & 16 & .6688 & .6903 & .6823 & $\left\{\begin{array}{l}11 / 16 \text { in } \\
17.5 \mathrm{~mm}_{-}\end{array}\right.$ & $\begin{array}{l}.6875 \\
.6890\end{array}$ & $\begin{array}{l}77 \\
75\end{array}$ \\
\hline $7 / 8--$ & 14 & .7822 & .8062 & .7977 & $\left\{\begin{array}{l}51 / 64 \mathrm{in}_{\ldots} \\
20.5 \mathrm{~mm}_{-}\end{array}\right.$ & $\begin{array}{l}.7969 \\
.8071\end{array}$ & $\begin{array}{l}84 \\
73\end{array}$ \\
\hline $1 \ldots$ & 14 & .9072 & .9312 & .9227 & $23.5 \mathrm{~mm}_{\ldots} \ldots$ & .9252 & 81 \\
\hline $11 / 8$ & 12 & 1. 0167 & 1. 0438 & 1. 0348 & $26.5 \mathrm{~mm}_{\ldots} \ldots$ & 1. 0433 & 75 \\
\hline $11 / 4$ & 12 & 1. 1417 & 1. 1688 & 1. 1598 & $29.5 \mathrm{~mm}_{-\ldots} \ldots$ & 1. 1614 & 82 \\
\hline $13 / 8$ & 12 & 1. 2667 & 1. 2938 & 1. 2848 & $\left\{\begin{array}{l}9 / 32 \text { in } \\
19 / 64 \text { in }\end{array}\right.$ & $\begin{array}{l}\text { 1. } 2812 \\
\text { 1. } 2969\end{array}$ & $\begin{array}{r}8 \\
72\end{array}$ \\
\hline $1 \frac{1}{2-}$ & 12 & 1. 3917 & 1. 4188 & 1. 4098 & $36 \mathrm{~mm}_{\ldots} \ldots$ & 1. 4173 & 76 \\
\hline
\end{tabular}


TaBle 123 (A).-Sizes of tap drills

[American National 8-pitch-thread series]

\begin{tabular}{|c|c|c|c|c|c|c|c|}
\hline \multirow[b]{2}{*}{$\begin{array}{l}\text { Size of } \\
\text { thread }\end{array}$} & \multirow[b]{2}{*}{$\begin{array}{l}\text { Threads } \\
\text { per in. }\end{array}$} & \multicolumn{3}{|c|}{ Minor diameter of nut } & \multicolumn{3}{|c|}{$\begin{array}{l}\text { Stock drills and corresponding percentage of } \\
\text { basic thread depth }\end{array}$} \\
\hline & & Basic & Maximum & Minimum & Nominal size & Diameter & $\begin{array}{l}\text { Percentage } \\
\text { of depth of } \\
\text { basic } \\
\text { thread }\end{array}$ \\
\hline 1 & 8 & $\begin{array}{l}\text { Inch } \\
0.8376\end{array}$ & $\begin{array}{l}\text { Inch } \\
0.8795\end{array}$ & $\begin{array}{l}\text { Inch } \\
0.8647\end{array}$ & $\left\{\begin{array}{l}22 \mathrm{~mm}_{--} \\
7 \% 8 \mathrm{in}-\ldots\end{array}\right.$ & $\begin{aligned} & \text { Inch } \\
& 0.8661 \\
& .8750\end{aligned}$ & $\begin{array}{l}82 \\
77\end{array}$ \\
\hline $11 / 8$ & 8 & .9626 & 1. 0045 & .9897 & $\left\{\begin{array}{l}1 \mathrm{in} \\
25.5 \mathrm{~mm}\end{array}\right.$ & $\begin{array}{l}\text { 1. } 0000 \\
\text { 1. } 0039\end{array}$ & $\begin{array}{l}77 \\
75\end{array}$ \\
\hline $11 / 4$ & 8 & 1. 0876 & 1. 1295 & 1. 1147 & $\left\{\begin{array}{l}28.5 \mathrm{~mm} \\
11 / 8 \text { in } \ldots\end{array}\right.$ & $\begin{array}{l}\text { 1. } 1220 \\
\text { 1. } 1250\end{array}$ & $\begin{array}{l}79 \\
77\end{array}$ \\
\hline $13 / 8$ & 8 & 1. 2126 & 1. 2545 & 1. 2397 & $\left\{\begin{array}{l}31.5 \mathrm{~mm}_{-} \\
11 / 4 \mathrm{in} \ldots\end{array}\right.$ & $\begin{array}{l}\text { 1. } 2402 \\
\text { 1. } 2500\end{array}$ & $\begin{array}{l}83 \\
77\end{array}$ \\
\hline $11 / 2$ & 8 & 1. 3376 & 1. 3795 & 1. 3647 & $\left\{\begin{array}{l}13 / 8 \mathrm{in}_{\ldots} \ldots \\
35 \mathrm{~mm}\end{array}\right.$ & $\begin{array}{l}\text { 1. } 3750 \\
\text { 1. } 3780\end{array}$ & $\begin{array}{l}77 \\
75\end{array}$ \\
\hline $15 / 8$ & 8 & 1. 4626 & 1. 5045 & 1. 4897 & $\left\{\begin{array}{l}38 \mathrm{~mm} \ldots \\
1 / 2 \mathrm{in} \ldots\end{array}\right.$ & $\begin{array}{l}\text { 1. } 4961 \\
\text { 1. } 5000\end{array}$ & $\begin{array}{l}79 \\
77\end{array}$ \\
\hline $13 / 4$ & 8 & 1. 5876 & 1. 6295 & 1. 6147 & $15 / 8$ in $\ldots \ldots$ & 1. 6250 & 77 \\
\hline $17 / 8$ & 8 & 1. 7126 & 1. 7545 & 1. 7397 & $\left\{\begin{array}{l}13 / 4 \mathrm{in}_{1} \\
44.5 \mathrm{~mm}\end{array}\right.$ & $\begin{array}{l}\text { 1. } 7500 \\
\text { 1. } 7520\end{array}$ & $\begin{array}{l}77 \\
76\end{array}$ \\
\hline 2 & 8 & 1. 8376 & 1. 8795 & 1. 8647 & $\left\{\begin{array}{l}47.5 \mathrm{~mm}_{\ldots} \\
17 / 8 \mathrm{in} \ldots\end{array}\right.$ & $\begin{array}{l}\text { 1. } 8701 \\
\text { 1. } 8750\end{array}$ & $\begin{array}{l}80 \\
77\end{array}$ \\
\hline $21 / 8$ & 8 & 1. 9626 & 2. 0045 & 1. 9897 & 2 in $\ldots$ & 2. 0000 & 77 \\
\hline $21 / 4$ & 8 & 2. 0876 & 2. 1295 & 2. 1147 & $\left\{\begin{array}{l}21 / 8 \text { in } \\
54 \mathrm{~mm}\end{array}\right.$ & $\begin{array}{l}\text { 2. } 1250 \\
\text { 2. } 1260\end{array}$ & $\begin{array}{l}77 \\
76\end{array}$ \\
\hline $21 / 2$ & 8 & 2. 3376 & 2. 3795 & 2. 3647 & $23 / 8$ in $\ldots$ & 2. 3750 & 77 \\
\hline $23 / 4$ & 8 & 2. 5876 & 2. 6295 & 2. 6147 & $\left\{\begin{array}{l}66.5 \mathrm{~mm} \\
25 / 8 \text { in } \ldots\end{array}\right.$ & $\begin{array}{l}\text { 2. } 6181 \\
\text { 2. } 6250\end{array}$ & $\begin{array}{l}81 \\
77\end{array}$ \\
\hline 3 & 8 & 2. 8376 & 2. 8795 & 2. 8647 & $\left\{\begin{array}{l}73 \mathrm{~mm} \\
27 / 8 \mathrm{in} \ldots\end{array}\right.$ & $\begin{array}{l}\text { 2. } 8740 \\
\text { 2. } 8750\end{array}$ & $\begin{array}{l}78 \\
77\end{array}$ \\
\hline $31 / 4$ & 8 & 3. 0876 & 3. 1295 & 3. 1147 & $31 / 8$ in $\ldots$ & 3. 1250 & 77 \\
\hline $31 / 2$ & 8 & 3. 3376 & 3. 3795 & 3. 3647 & $33 / 8$ in $\ldots$ & 3. 3750 & 77 . \\
\hline
\end{tabular}

1 Present Army Ordnance practice follows Handbook Hi25 and the mimeographed Supplement to Handbook $\mathrm{H} 28$ in the maximum minor diameters of nuts. 
Table 123 (B).-Sizes of tap drills

[American National 12-pitch thread series]

\begin{tabular}{|c|c|c|c|c|c|c|c|}
\hline \multirow[b]{2}{*}{$\begin{array}{l}\text { Size of } \\
\text { thread }\end{array}$} & \multirow[b]{2}{*}{$\begin{array}{l}\text { Threads } \\
\text { per in. }\end{array}$} & \multicolumn{3}{|c|}{ Minor diameter of nut } & \multicolumn{3}{|c|}{$\begin{array}{l}\text { Stock drilis and corresponding percentage of } \\
\text { basic thread depth } 1\end{array}$} \\
\hline & & Basic & Maximum & Minimum & Nominal size & Diameter & $\begin{array}{l}\text { Percentage } \\
\text { of depth of } \\
\text { basic } \\
\text { thread }\end{array}$ \\
\hline $1 / 2 \ldots$ & 12 & $\begin{array}{c}\text { Inch } \\
\text { 0. } 3917\end{array}$ & $\begin{array}{c}\text { Inch } \\
0.4225\end{array}$ & $\begin{array}{c}\text { Inch } \\
\text { 0. } 4098\end{array}$ & $\left\{\begin{array}{l}\mathrm{Z}^{3} \\
10.5 \mathrm{~mm}^{3} \\
27 / 64 \mathrm{in}_{--}\end{array}\right.$ & $\begin{array}{r}\text { Inch } \\
0.4130 \\
.4134 \\
.4219\end{array}$ & $\begin{array}{l}80 \\
80 \\
72\end{array}$ \\
\hline $9 / 16-$ & 12 & . 4542 & .4850 & .4723 & $\left\{\begin{array}{l}12 \mathrm{~mm}^{3}-- \\
31 / 64 \mathrm{in}_{-}\end{array}\right.$ & $\begin{array}{l}.4724 \\
.4844\end{array}$ & $\begin{array}{l}83 \\
72\end{array}$ \\
\hline $5 / 8$ & 12 & .5167 & .5438 & .5348 & $\left\{\begin{array}{l}13.5 \mathrm{~mm}_{-} \\
35 / 64 \mathrm{in}\end{array}\right.$ & $\begin{array}{l}.5315 \\
.5469\end{array}$ & $\begin{array}{l}86 \\
72\end{array}$ \\
\hline $11 / 16-$ & 12 & .5792 & .6063 & .5973 & $\left\{\begin{array}{l}19 / 32 \text { in } \\
39 / 64 \text { in.-- }\end{array}\right.$ & $\begin{array}{l}.5938 \\
.6094\end{array}$ & $\begin{array}{l}87 \\
72\end{array}$ \\
\hline $3 / 4$ & 12 & .6417 & .6688 & .6598 & $\left\{\begin{array}{l}21 / 32 \text { in } \ldots \\
17 \mathrm{~mm} \ldots\end{array}\right.$ & $\begin{array}{l}.6562 \\
.6693\end{array}$ & $\begin{array}{l}87 \\
75\end{array}$ \\
\hline $13 / 16 \ldots$ & 12 & .7042 & .7313 & .7223 & $18.5 \mathrm{~mm}$ & .7283 & 78 \\
\hline $7 / 8 \ldots$ & 12 & .7667 & .7938 & .7848 & $20 \mathrm{~mm}$ & .7874 & 81 \\
\hline $15 / 16 \ldots$ & 12 & .8292 & .8563 & .8473 & $\left\{\begin{array}{l}21.5 \mathrm{~mm}_{\ldots} \ldots \\
55 / 64 \mathrm{in} \ldots\end{array}\right.$ & $\begin{array}{l}.8465 \\
.8594\end{array}$ & $\begin{array}{l}84 \\
72\end{array}$ \\
\hline $1 \ldots$ & 12 & .8917 & .9188 & 9098 & $\left\{\begin{array}{l}29 / 32 \text { in } \\
59 / 64 \text { in }\end{array}\right.$ & $\begin{array}{l}.9062 \\
.9219\end{array}$ & $\begin{array}{l}87 \\
72\end{array}$ \\
\hline $11 / 16 \ldots$ & 12 & .9542 & . 9813 & .9723 & $\left\{\begin{array}{l}31 / 32 \text { in } \\
25 \mathrm{~mm}_{\ldots}\end{array}\right.$ & $\begin{array}{l}.9687 \\
.9843\end{array}$ & $\begin{array}{l}87 \\
72\end{array}$ \\
\hline $11 / 8 \ldots$ & 12 & 1. 0167 & 1. 0438 & 1. 0348 & $26.5 \mathrm{~mm}_{\ldots} \ldots$ & 1. 0433 & 75 \\
\hline $13 / 16--$ & 12 & 1. 0792 & 1. 1063 & 1. 0973 & $28 \mathrm{~mm}_{-}--$ & 1. 1024 & 79 \\
\hline $11 / 4 \ldots$ & 12 & 1. 1417 & 1. 1688 & 1. 1598 & $29.5 \mathrm{~mm} \ldots$ & 1. 1614 & 82 \\
\hline $15 / 16--$ & 12 & 1. 2042 & 1. 2313 & 1. 2223 & $\begin{cases}31 & m m \\
1 & 15 / 64\end{cases}$ & $\begin{array}{l}\text { 1. } 2205 \\
\text { 1. } 2344\end{array}$ & $\begin{array}{l}85 \\
72\end{array}$ \\
\hline $13 / 8 \ldots$ & 12 & 1. 2667 & 1. 2938 & 1. 2848 & $\begin{cases}1 & 9 / 32 \\
1 & 19 / 64\end{cases}$ & $\begin{array}{l}\text { 1. } 2812 \\
\text { 1. } 2969\end{array}$ & $\begin{array}{l}87 \\
72\end{array}$ \\
\hline $17 / 16 \ldots$ & 12 & 1. 3292 & 1. 3563 & 1. 3473 & $\begin{cases}1 & 11 / 32 \\
34.5 & \mathrm{~mm}\end{cases}$ & $\begin{array}{l}\text { 1. } 3438 \\
\text { 1. } 3583\end{array}$ & $\begin{array}{l}87 \\
78\end{array}$ \\
\hline
\end{tabular}

1 Sizes in italics are not within the specified limits for minor diameter of nut. 3 See footnote at end of table 123 (D). 
Table 123 (B).-Sizes of tap drills-Continued

[American National 12-pitch thread series]

\begin{tabular}{|c|c|c|c|c|c|c|c|}
\hline \multirow[b]{2}{*}{$\begin{array}{l}\text { Size of } \\
\text { thread }\end{array}$} & \multirow[b]{2}{*}{$\begin{array}{l}\text { Threads } \\
\text { per in. }\end{array}$} & \multicolumn{3}{|c|}{ Minor diameter of nut } & \multicolumn{3}{|c|}{$\begin{array}{l}\text { Stock drills and corresponding percentage of } \\
\text { basic thread depth } 1\end{array}$} \\
\hline & & Basic & Maximum & Minimum & Nominal size & Diameter & $\begin{array}{l}\text { Percentage } \\
\text { of depth of } \\
\text { basic } \\
\text { thread }\end{array}$ \\
\hline $11 / 2$ & 12 & $\begin{array}{l}\text { Inch } \\
\text { 1. } 3917\end{array}$ & $\begin{array}{c}\text { Inch } \\
\text { 1. } 4188\end{array}$ & $\begin{array}{c}\text { Inch } \\
\text { 1. } 4098\end{array}$ & $36 \mathrm{~mm}_{-.-}$ & $\begin{array}{c}\text { Inch } \\
\text { 1. } 4173\end{array}$ & 76 \\
\hline $15 / 8 \ldots$ & 12 & 1. 5167 & 1. 5438 & 1. 5348 & $39 \mathrm{~mm}$ & 1. 5354 & 83 \\
\hline $13 / 4$ & 12 & 1. 6417 & 1. 6688 & 1. 6598 & $\begin{cases}1 & 21 / 32 \\
1 & 43 / 64 \\
\text { in }\end{cases}$ & $\begin{array}{l}\text { 1. } 6562 \\
\text { 1. } 6719\end{array}$ & $\begin{array}{l}87 \\
72\end{array}$ \\
\hline $17 / 8 \ldots$ & 12 & 1. 7667 & 1. 7938 & 1. 7848 & $45.5 \mathrm{~mm}_{\ldots} \ldots$ & 1. 7913 & 77 \\
\hline 2 & 12 & 1. 8917 & 1. 9188 & 1. 9098 & 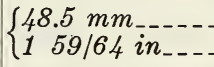 & $\begin{array}{l}\text { 1. } 9094 \\
\text { 1. } 9219\end{array}$ & $\begin{array}{l}84 \\
72\end{array}$ \\
\hline $21 / 8$ & 12 & 2. 0167 & 2. 0438 & 2. 0348 & $\left\{\begin{array}{lll}2 & 1 / 32 & \text { in } \\
2 & 3 / 64 & \text { in }--\end{array}\right.$ & $\begin{array}{l}\text { 2. } 0312 \\
\text { 2. } 0469\end{array}$ & $\begin{array}{l}87 \\
72\end{array}$ \\
\hline $21 / 4$ & 12 & 2. 1417 & 2. 1688 & 2. 1598 & $55 \mathrm{~mm}_{\ldots}$ & 2. 1654 & 78 \\
\hline $23 / 8$ & 12 & 2. 2667 & 2. 2938 & 2. 2848 & $\begin{cases}58 & \mathrm{~mm} \\
2 & 19 / 64 \\
\mathrm{in}\end{cases}$ & $\begin{array}{l}\text { 2. } 2835 \\
\text { 2. } 2969\end{array}$ & $\begin{array}{l}85 \\
72\end{array}$ \\
\hline $21 / 2-$ & 12 & 2. 3917 & 2. 4188 & 2. 4098 & $\left\{\begin{array}{l}213 / 32 \text { in } \\
61.5 \mathrm{~mm}\end{array}\right.$ & $\begin{array}{l}\text { 2. } 4062 \\
\text { 2. } 4213\end{array}$ & $\begin{array}{l}87 \\
73\end{array}$ \\
\hline $25 / 8$ & 12 & 2. 5167 & 2. 5438 & 2. 5348 & $64.5 \mathrm{~mm} \ldots$ & 2. 5394 & 79 \\
\hline $23 / 4 \ldots$ & 12 & 2. 6417 & 2. 6688 & 2. 6598 & $\left\{\begin{array}{lll}67.5 & \mathrm{~mm}_{-} \\
2 & 43 / 64 & \mathrm{in}_{-}\end{array}\right.$ & $\begin{array}{l}\text { 2. } 6575 \\
\text { 2. } 6719\end{array}$ & $\begin{array}{l}85 \\
72\end{array}$ \\
\hline $27 / 8--$ & 12 & 2. 7667 & 2. 7938 & 2. 7848 & $\left\{\begin{array}{lll}2 & 25 / 32 & \mathrm{in} \\
71 & \mathrm{~mm}\end{array}\right.$ & $\begin{array}{l}\text { 2. } 7812 \\
\text { 2. } 7953\end{array}$ & $\begin{array}{l}87 \\
74\end{array}$ \\
\hline $3 \ldots \ldots$ & 12 & 2. 8917 & 2. 9188 & 2. 9098 & $74 \mathrm{~mm}$ & 2. 9134 & 80 \\
\hline $31 / 8 \ldots$ & 12 & 3. 0167 & 3. 0438 & 3. 0348 & $\left\{\begin{array}{lll}3 & 1 / 32 & i n \\
3 & 1 / 16 & \text { in }\end{array}\right.$ & $\begin{array}{l}\text { 3. } 0312 \\
\text { 3. } 0625\end{array}$ & $\begin{array}{l}87 \\
58\end{array}$ \\
\hline $31 / 4-\ldots$ & 12 & 3. 1417 & 3. 1688 & 3. 1598 & $\left\{\begin{array}{lll}3 & 5 / 32 & i n \\
3 & 3 / 16 & \text { in }\end{array}\right.$ & $\begin{array}{l}\text { 3. } 1562 \\
\text { 3. } 1875\end{array}$ & $\begin{array}{l}87 \\
58\end{array}$ \\
\hline $33 / 8--$ & 12 & 3. 2667 & 3. 2938 & 3. 2848 & $\left\{\begin{array}{lll}3 & 9 / 32 & \text { in } \\
3 & 5 / 16 & \text { in }\end{array}\right.$ & $\begin{array}{l}\text { 3. } 2812 \\
\text { 3. } 3125\end{array}$ & $\begin{array}{l}87 \\
58\end{array}$ \\
\hline $31 / 2$ & 12 & 3. 3917 & 3. 4188 & 3. 4098 & $3 \mathrm{7} / 16 \mathrm{in}_{\ldots}$ & 3. 4375 & 58 \\
\hline
\end{tabular}

1 Sizes in italies are not within the specified limits for minor diameter of nut. 
TABLE 123 (C).- Sizes of tap drills

[American National 16-pitch-thread series]

\begin{tabular}{|c|c|c|c|c|c|c|c|}
\hline \multirow[b]{2}{*}{$\begin{array}{l}\text { Size of } \\
\text { thread }\end{array}$} & \multirow[b]{2}{*}{$\begin{array}{c}\text { Threads per } \\
\text { inch }\end{array}$} & \multicolumn{3}{|c|}{ Minor diameter of nut } & \multicolumn{3}{|c|}{$\begin{array}{l}\text { Stock drills and corresponding percentage of } \\
\text { basic thread depth }\end{array}$} \\
\hline & & Basic & Maximum 1 & Minimum & Nominal size & Diameter & $\begin{array}{l}\text { Percentage } \\
\text { of depth } \\
\text { of basic } \\
\text { thread }\end{array}$ \\
\hline $3 / 4 \ldots$ & 16 & $\begin{array}{c}\text { Inch } \\
0.6688\end{array}$ & $\begin{array}{c}\text { Inch } \\
0.6903\end{array}$ & $\begin{array}{c}\text { Inch } \\
0.6823\end{array}$ & $\left\{\begin{array}{l}11 / 16 \mathrm{in} \ldots \ldots \\
17.5 \mathrm{~mm}\end{array}\right.$ & $\begin{array}{r}\text { Inch } \\
0.6875 \\
.6890\end{array}$ & $\begin{array}{l}77 \\
.75\end{array}$ \\
\hline $13 / 16 \ldots$ & 16 & .7313 & .7528 & .7448 & $\left\{\begin{array}{l}19 \mathrm{~mm}_{3} \ldots \\
3 / 4 \mathrm{in} \ldots\end{array}\right.$ & $\begin{array}{l}.7480 \\
.7500\end{array}$ & $\begin{array}{l}79 \\
77\end{array}$ \\
\hline $7 / 8 \ldots$ & 16 & .7938 & .8153 & .8073 & $13 / 16$ in & .8125 & 77 \\
\hline $15 / 16 \ldots$ & 16 & .8563 & .8778 & 8698 & $7 / 8$ in $\ldots$ & .8750 & 77 \\
\hline $1 \ldots \ldots$ & 16 & .9188 & .9403 & .9323 & $15 / 16$ in $\ldots$ & .9375 & 77 \\
\hline $11 / 16 \ldots$ & 16 & .9813 & 1. 0028 & .9948 & 1 in $\ldots$ & 1. 0000 & 77 \\
\hline $11 / 8 \ldots$ & 16 & 1. 0438 & 1. 0653 & 1. 0573 & $\left\{\begin{array}{l}11 / 16 \text { in } \\
27 \mathrm{~mm}_{-}-\ldots\end{array}\right.$ & $\begin{array}{l}\text { 1. } 0625 \\
\text { 1. } 0630\end{array}$ & $\begin{array}{l}77 \\
76\end{array}$ \\
\hline $13 / 16$ & 16 & 1. 1063 & 1. 1278 & 1. 1198 & $\left\{\begin{array}{l}28.5 \mathrm{~mm} \\
11 / \mathrm{s} \text { in }\end{array}\right.$ & $\begin{array}{l}\text { 1. } 1220 \\
\text { 1. } 1250\end{array}$ & $\begin{array}{l}81 \\
77\end{array}$ \\
\hline $11 / 4 \ldots$ & 16 & 1. 1688 & 1. 1903 & 1. 1823 & $13 / 16$ in & 1. 1875 & 77 \\
\hline $15 / 16---$ & 16 & 1. 2313 & 1. 2528 & 1. 2448 & $1 \frac{1}{1} 1$ in & 1. 2500 & 77 \\
\hline $13 / 8 \ldots$ & 16 & 1. 2938 & 1. 3153 & 1. 3073 & $15 / 16$ in & 1. 3125 & 77 \\
\hline $17 / 16--$ & 16 & 1. 3563 & 1. 3778 & 1. 3698 & $13 / 8$ in & 1. 3750 & 77 \\
\hline $11 / 2$ & 16 & 1. 4188 & 1. 4403 & 1. 4323 & $\left\{\begin{array}{l}36.5 \mathrm{~mm} \\
17 / 16 \text { in }\end{array}\right.$ & $\begin{array}{l}\text { 1. } 4370 \\
\text { 1. } 4375\end{array}$ & $\begin{array}{l}78 \\
77\end{array}$ \\
\hline $1 \% / 16--$ & 16 & 1. 4813 & 1. 5028 & 1. 4948 & $\left\{\begin{array}{r}38 \mathrm{~mm}_{1} \ldots \\
1 / 2 \mathrm{in}\end{array}\right.$ & $\begin{array}{l}\text { 1. } 4961 \\
\text { 1. } 5000\end{array}$ & $\begin{array}{l}82 \\
77\end{array}$ \\
\hline $15 / 8$ & 16 & 1. 5438 & 1. 5653 & 1. 5573 & $19 / 16$ in & 1. 5625 & 77 \\
\hline $111 / 16--$ & 16 & 1. 6063 & 1. 6278 & 1. 6198 & $15 / 8$ in & 1. 6250 & 77 \\
\hline $13 / 4 \ldots$ & 16 & 1. 6688 & 1. 6903 & 1. 6823 & $111 / 16$ in & 1. 6875 & 77 \\
\hline $113 / 16$ & 16 & 1. 7313 & 1. 7528 & 1. 7448 & $\left\{\begin{array}{l}13 / 4 \mathrm{in} \\
44.5 \mathrm{~mm}\end{array}\right.$ & $\begin{array}{l}\text { 1. } 7500 \\
\text { 1. } 7520\end{array}$ & $\begin{array}{l}77 \\
75\end{array}$ \\
\hline
\end{tabular}

1 Present Army ordnance practice follows Handbook H25 and the mimeographed Supplement to Handbook $\mathrm{H} 28$ in the maximum minor diameters of nuts. 
TABLE 123 (C).-Sizes of tap drills-Continued

[American National 16-pitch-thread series]

\begin{tabular}{|c|c|c|c|c|c|c|c|}
\hline \multirow[b]{2}{*}{$\begin{array}{l}\text { Size of } \\
\text { thread }\end{array}$} & \multirow[b]{2}{*}{$\begin{array}{l}\text { Threads } \\
\text { per in. }\end{array}$} & \multicolumn{3}{|c|}{ Minor diameter of nut } & \multicolumn{3}{|c|}{$\begin{array}{l}\text { Stock drills and corresponding percentage of } \\
\text { basic thread depth }\end{array}$} \\
\hline & & Basic & Maximum & Minimum & Nominal size & Diameter & $\begin{array}{l}\text { Percentage } \\
\text { of depth of } \\
\text { basic } \\
\text { thread }\end{array}$ \\
\hline $17 / 8$ & 16 & $\begin{array}{c}\text { Inch } \\
\text { 1. } 7938\end{array}$ & $\begin{array}{c}\text { Inch } \\
\text { 1. } 8153\end{array}$ & $\begin{array}{c}\text { Inch } \\
\text { 1. } 8073\end{array}$ & $\left\{\begin{array}{l}46 \mathrm{~mm}_{-} \\
1^{13 / 16} \text { in-- }\end{array}\right.$ & $\begin{array}{c}\text { Inch } \\
\text { 1. } 8110 \\
\text { 1. } 8125\end{array}$ & $\begin{array}{l}79 \\
77\end{array}$ \\
\hline $115 / 16--$ & 16 & 1. 8563 & 1. 8778 & 1. 8698 & $\left\{\begin{array}{l}47.5 \mathrm{~mm}_{-} \\
178 \mathrm{in} .-\end{array}\right.$ & $\begin{array}{l}\text { 1. } 8701 \\
\text { 1. } 8750\end{array}$ & $\begin{array}{l}83 \\
77\end{array}$ \\
\hline $2 \ldots \ldots$ & 16 & 1. 9188 & 1. 9403 & 1. 9323 & $1 \frac{15}{16}$ in & 1. 9375 & 77 \\
\hline $21 / 16--$ & 16 & 1. 9813 & 2. 0028 & 1. 9948 & 2 in $\ldots$ & 2. 0000 & 77 \\
\hline $2 \frac{1}{8--}$ & 16 & 2. 0438 & 2. 0653 & 2. 0573 & $2^{1 / 16}$ in $\ldots$ & 2. 0625 & 77 \\
\hline $23 / 16-$ & 16 & 2. 1063 & 2. 1278 & 2. 1198 & $\left\{\begin{array}{l}21 / 8 \mathrm{in}_{----} \\
54 \mathrm{~mm}_{--}\end{array}\right.$ & $\begin{array}{l}\text { 2. } 1250 \\
\text { 2. } 1260\end{array}$ & $\begin{array}{l}77 \\
76\end{array}$ \\
\hline $21 / 4--$ & 16 & 2. 1688 & 2. 1903 & 2. 1823 & $\left\{\begin{array}{l}55.5 \mathrm{~mm}_{--} \\
23 / 16 \mathrm{in}_{---}\end{array}\right.$ & $\begin{array}{l}\text { 2. } 1850 \\
\text { 2. } 1875\end{array}$ & $\begin{array}{l}80 \\
77\end{array}$ \\
\hline $25 / 10--$ & 16 & 2. 2313 & 2. 2528 & 2. 2448 & $21 / 4$ in & 2. 2500 & 77 \\
\hline $23 / 8$ & 16 & 2. 2938 & 2. 3153 & 2. 3073 & $25 / 16$ in & 2. 3125 & 77 \\
\hline $27 / 16---$ & 16 & 2. 3563 & 2. 3778 & 2. 3698 & $23 / 8$ in & 2. 3750 & 77 \\
\hline $21 / 2 \ldots$ & 16 & 2. 4188 & 2. 4403 & 2. 4323 & $27 / 16$ in $\ldots$ & 2. 4375 & 77 \\
\hline $25 \% 8$ & 16 & 2. 5438 & 2. 5653 & 2. 5573 & $\left\{\begin{array}{l}65 \mathrm{~mm}_{-} \\
2 \% 16 \mathrm{in}_{---}\end{array}\right.$ & $\begin{array}{l}\text { 2. } 5590 \\
\text { 2. } 5625\end{array}$ & $\begin{array}{l}81 \\
77\end{array}$ \\
\hline $23 / 4-\ldots$ & 16 & 2. 6688 & 2. 6903 & 2. 6823 & $2^{11 / 16}$ in $\ldots$ & 2. 6875 & 77 \\
\hline $27 / 8-$ & 16 & 2. 7938 & 2. 8153 & 2. 8073 & $\left\{\begin{array}{l}2^{13} / 16 \text { in } \\
71.5 \mathrm{~mm}\end{array}\right.$ & $\begin{array}{l}\text { 2. } 8125 \\
\text { 2. } 8150\end{array}$ & $\begin{array}{l}77 \\
74\end{array}$ \\
\hline $3 \ldots$ & 16 & 2. 9188 & 2. 9403 & 2. 9323 & $\left\{\begin{array}{l}74.5 \mathrm{~mm} \\
2^{15} / 16 \text { in } \ldots\end{array}\right.$ & $\begin{array}{l}\text { 2. } 9331 \\
\text { 2. } 9375\end{array}$ & $\begin{array}{l}82 \\
77\end{array}$ \\
\hline $31 / 8 \ldots$ & 16 & 3. 0438 & 3. 0653 & 3. 0573 & $31 / 16$ in $\ldots$ & 3. 0625 & 77 \\
\hline $31 / 4----$ & 16 & 3. 1688 & 3. 1903 & 3. 1823 & $33 / 16$ in & 3. 1875 & 77 \\
\hline $33 / 8 \ldots$ & 16 & 3. 2938 & 3. 3153 & 3. 3073 & $35 / 16$ in & 3. 3125 & 77 \\
\hline $31 / 2 \ldots$ & 16 & 3. 4188 & 3. 4403 & 3. 4323 & $37 / 16$ in $\ldots$ & 3. 4375 & 77 \\
\hline
\end{tabular}


TABLE 123 (D).-Sizes of tap drills

[American National extra-fine-thread series]

\begin{tabular}{|c|c|c|c|c|c|c|c|}
\hline \multirow[b]{2}{*}{$\begin{array}{l}\text { Size of } \\
\text { thread }\end{array}$} & \multirow[b]{2}{*}{$\begin{array}{l}\text { Threads } \\
\text { per inch }\end{array}$} & \multicolumn{3}{|c|}{ Minor diameter of nut } & \multicolumn{3}{|c|}{$\begin{array}{l}\text { Stock drills and corresponding percentage of } \\
\text { basic thread depth } 1\end{array}$} \\
\hline & & Basic & Maximum 2 & Minimum & Nominal size & Diameter & $\begin{array}{l}\text { Percentage } \\
\text { of depth of } \\
\text { basic } \\
\text { thread }\end{array}$ \\
\hline $1 / 4-$ & 32 & $\begin{array}{c}\text { Inch } \\
\text { 0. } 2094\end{array}$ & $\begin{array}{c}\text { Inch } \\
0.2208\end{array}$ & $\begin{array}{c}\text { Inch } \\
0.2162\end{array}$ & $\left\{\begin{array}{l}5.5 \mathrm{~mm} .^{3}- \\
7 / 32 \mathrm{in}-{ }_{-}- \\
5.6 \mathrm{~mm} .^{3}\end{array}\right.$ & $\begin{aligned} \text { Inch } \\
0.2165 \\
.2188 \\
.2205\end{aligned}$ & $\begin{array}{l}83 \\
77 \\
73\end{array}$ \\
\hline $5 / 16-\ldots$ & 32 & .2719 & .2833 & .2787 & $\left\{\begin{array}{l}7.1 \mathrm{~mm} .^{3}-\ldots \\
\mathrm{K}^{3}{ }^{3} \mathrm{in}_{32} \mathrm{in}\end{array}\right.$ & $\begin{array}{l}.2795 \\
.2810 \\
.2812\end{array}$ & $\begin{array}{l}81 \\
77 \\
77\end{array}$ \\
\hline $3 / 8 \ldots$ & 32 & .3344 & .3458 & .3412 & $\left\{\begin{array}{l}8.7 \mathrm{~mm} .^{3} \ldots \\
11 / 32 \mathrm{in} \\
8.75 \mathrm{~mm} .^{3} \ldots\end{array}\right.$ & $\begin{array}{l}.3425 \\
.3438 \\
.3445\end{array}$ & $\begin{array}{l}80 \\
77 \\
75\end{array}$ \\
\hline $7 / 16 \ldots$ & 28 & .3911 & .4041 & .3988 & $\left\{\begin{array}{l}X \\
Y^{3}-\cdots\end{array}\right.$ & $\begin{array}{l}.3970 \\
.4040\end{array}$ & $\begin{array}{l}87 \\
72\end{array}$ \\
\hline $1 / 2 \ldots$ & 28 & .4536 & .4666 & .4613 & $\begin{cases}29 / 64 & \text { in } \\
15 / 32 & \text { in }\end{cases}$ & $\begin{array}{l}.4531 \\
.4687\end{array}$ & $\begin{array}{r}101 \\
67\end{array}$ \\
\hline $9 / 16-$ & 24 & .5084 & .5235 & .5174 & $\begin{cases}33 / 64 & \text { in } \\
17 / 32 & \text { in }\end{cases}$ & $\begin{array}{l}.5156 \\
.5312\end{array}$ & $\begin{array}{l}87 \\
58\end{array}$ \\
\hline $5 / 8--$ & 24 & .5709 & .5860 & .5799 & $\left\{\begin{array}{l}37 / 64 \text { in } \ldots \ldots \\
15 \mathrm{~mm}_{\ldots} \ldots \ldots\end{array}\right.$ & $\begin{array}{l}.5781 \\
.5906\end{array}$ & $\begin{array}{l}87 \\
64\end{array}$ \\
\hline $11 / 16$ & 24 & .6334 & .6485 & .6424 & $\left\{\begin{array}{l}41 / 64 \text { in } \\
16.5 \mathrm{~mm}_{\ldots} \ldots\end{array}\right.$ & $\begin{array}{l}.6406 \\
.6496\end{array}$ & $\begin{array}{l}87 \\
70\end{array}$ \\
\hline $3 / 4+-$ & 20 & .6850 & .7027 & .6959 & $\left\{\begin{array}{l}17.5 \mathrm{~mm}_{\ldots} \ldots \ldots \\
4564 \mathrm{in} \ldots\end{array}\right.$ & $\begin{array}{l}.6890 \\
.7031\end{array}$ & $\begin{array}{l}94 \\
72\end{array}$ \\
\hline $13 / 16$ & 20 & .7475 & .7652 & .7584 & $\left\{\begin{array}{l}3 / 4 \text { in } \\
4964 \text { in } \ldots \ldots\end{array}\right.$ & $\begin{array}{l}.7500 \\
.7656\end{array}$ & $\begin{array}{l}96 \\
72\end{array}$ \\
\hline $7 / 8 \ldots$ & 20 & .8100 & .8277 & .8209 & $21 \mathrm{~mm}_{\ldots} \ldots$ & .8268 & 74 \\
\hline $15 / 16-\ldots$ & 20 & .8725 & .8902 & .8834 & $22.5 \mathrm{~mm}_{\ldots} \ldots$ & .8858 & 80 \\
\hline $1 \ldots$ & 20 & .9350 & .9527 & .9459 & $\left\{\begin{array}{l}24 \mathrm{~mm}_{\ldots} \ldots \ldots \\
61 / 64 \mathrm{in} \ldots \ldots\end{array}\right.$ & $\begin{array}{l}.9449 \\
.9531\end{array}$ & $\begin{array}{l}85 \\
72\end{array}$ \\
\hline $11 / 16 \ldots$ & 18 & .9903 & 1. 0099 & 1. 0024 & $25.5 \mathrm{~mm}_{\ldots} \ldots$ & 1. 0040 & 81 \\
\hline $11 / 8 \ldots$ & 18 & 1. 0528 & 1. 0724 & 1. 0649 & $\left\{\begin{array}{l}27 \mathrm{~mm}_{15} \ldots \ldots \\
1 \% \mathrm{in}_{\ldots}\end{array}\right.$ & $\begin{array}{l}\text { 1. } 0630 \\
\text { 1. } 0781\end{array}$ & $\begin{array}{l}86 \\
65\end{array}$ \\
\hline $13 / 16 \ldots$ & 18 & 1. 1153 & 1. 1349 & 1. 1274 & $\left\{\begin{array}{l}11 / 8 \text { in } \\
1 \% 64 \text { in }\end{array}\right.$ & $\begin{array}{l}\text { 1. } 1250 . \\
\text { 1. } 1406\end{array}$ & $\begin{array}{l}87 \\
65\end{array}$ \\
\hline $11 / 4 \ldots$ & 18 & 1. 1778 & 1. 1974 & 1. 1899 & $\left\{\begin{array}{l}13 / 16 \text { in } \\
30.5 \mathrm{~mm}_{\ldots}\end{array}\right.$ & $\begin{array}{l}\text { 1. } 1875 \\
\text { 1. } 2008\end{array}$ & $\begin{array}{l}87 \\
68\end{array}$ \\
\hline
\end{tabular}

1 Sizes in italics are not within the specified limits for minor diameter of nut.

2 Present Army ordnance practice follows Handbook H25 and the mimeographed Supplement to Hand book $\mathrm{H} 28$ in the maximum minor diameters of nuts.

3 These sizes are not included as standard in American Standard B 5.12-1940 for Twist Drills, Straight Shank, but are listed in the appendix thereto. 
TABLE 123 (D).-Sizes of tap drills-Continued

[American National extra-fine-thread series]

\begin{tabular}{|c|c|c|c|c|c|c|c|}
\hline \multirow[b]{2}{*}{$\begin{array}{l}\text { Size of } \\
\text { thread }\end{array}$} & \multirow[b]{2}{*}{$\begin{array}{l}\text { Threads } \\
\text { per inch }\end{array}$} & \multicolumn{3}{|c|}{ Minor diameter of nut } & \multicolumn{3}{|c|}{$\begin{array}{l}\text { Stock drills and corresponding percentage of } \\
\text { basic thread depth }\end{array}$} \\
\hline & & Basic & Maximum & Minimum & Nominal size & Diameter & $\begin{array}{l}\text { Percentage } \\
\text { of depth of } \\
\text { basic } \\
\text { thread }\end{array}$ \\
\hline $15 / 16 \ldots$ & 18 & $\begin{array}{l}\text { Inch } \\
\text { 1. } 2403\end{array}$ & $\begin{array}{l}\text { Inch } \\
\text { 1. } 2599\end{array}$ & $\begin{array}{l}\text { Inch } \\
\text { 1. } 2524\end{array}$ & $32 \mathrm{~mm}_{-}$. & $\begin{array}{l}\text { Inch } \\
\text { 1. } 2598\end{array}$ & 73 \\
\hline $13 / 8$ & 18 & 1. 3028 & 1. 3224 & 1. 3149 & $33.5 \mathrm{~mm} \ldots$ & 1. 3189 & 78 \\
\hline $17 / 16 \ldots$ & 18 & 1. 3653 & 1. 3849 & 1. 3774 & $35 \mathrm{~mm}_{\ldots}$ & 1. 3780 & 82 \\
\hline $11 / 2 \ldots$ & 18 & 1. 4278 & 1. 4474 & 1. 4399 & $\left\{\begin{array}{l}17 / 16 \text { in } \\
1^{29} / 64 \text { in }\end{array}\right.$ & $\begin{array}{l}\text { 1. } 4375 \\
\text { 1. } 4531\end{array}$ & $\begin{array}{l}87 \\
65\end{array}$ \\
\hline $1 \% 16 \ldots$ & 18 & 1. 4903 & 1. 5099 & 1. 5024 & $\left\{\begin{array}{l}11 / 2 \text { in } \\
1^{33} / 64\end{array}\right.$ & $\begin{array}{l}\text { 1. } 5000 \\
1.5156\end{array}$ & $\begin{array}{l}87 \\
65\end{array}$ \\
\hline $15 / 8 \ldots$ & 18 & 1. 5528 & 1. 5724 & 1. 5649 & $\left\{\begin{array}{l}19 / 16 \mathrm{in}_{\ldots} \\
40 \mathrm{~mm}\end{array}\right.$ & $\begin{array}{l}\text { 1. } 5625 \\
\text { 1. } 5748\end{array}$ & $\begin{array}{l}87 \\
70\end{array}$ \\
\hline $1^{11 / 16}$ & 18 & 1. 6153 & 1. 6349 & 1. 6274 & $41.5 \mathrm{~mm} \ldots$ & 1. 6339 & 74 \\
\hline $13 / 4$ & 16 & 1. 6688 & 1. 6903 & 1. 6823 & $1^{11} 1 / 16$ in $\ldots$ & 1. 6875 & 77 \\
\hline $2 \ldots$ & 16 & 1. 9188 & 1. 9403 & 1. 9323 & $115 / 16$ in & 1. 9375 & 77 \\
\hline
\end{tabular}

\section{LABELING}

12. Where the dimensions are to be guaranteed, the following form of statement on labels, invoices, catalogues, etc., is recommended:

The guarantees that for the respective classes of fit as identified or labeled, these screw threads conform to Commercial Standard CS24-43 as issued by the National Bureau of Standards of the U. S. Department of Commerce.

\section{EFFECTIVE DATE}

The standard is effective for new production from February 10, 1943.

\section{STANDING COMMITTEE}

The following individuals comprise the membership of the standing committee, which is to review, prior to circulation for acceptance, revisions proposed to keep the standard abreast of progress. Most organizations nominated their own representatives. Comment concerning the standard and suggestions for revision may be addressed to any member of the committee or to the Division of Trade Standards, National Bureau of Standards, which acts as secretary for the committee.

\section{Manufacturers:}

CARL W. Betrcher (Chairman), Eastern Machine Screw Corporation, New Haven, Conn.

J. J. Tomalis, American Screw Co., 21 Stevens Street, Providence, R. I. 
George S. Case, Lamson \& Sessions Co., 1975 W. 85th Street, Cleveland, OhioJ. S. Davey, Russell, Burdsall \& Ward Bolt \& Nut Co., Port Chester, N. Y.

J. H. Edmonds, Lebanon Plant, Bethlehem Steel Co., Lebanon, Pa.

H. C. Erdman, National Screw \& Mfg. Co., 2440 E. 75th St., Cleveland, Ohio W. C. Strewart, American Institute, Bolt, Nut and Rivet Mfrs., 1550 Hanna Bldg., Cleveland, Ohio.

F. P. Tisch, Pheoll Mfg. Co., 5700 Roosevelt Road, Chicago, Ill.

Charles C. Winter, Winter Bros. Co., Wrentham, Mass.

\section{Distributors:}

G. Cheston Carer, Carey Machinery \& Supply Co., 119 E. Lombard Street' Baltimore, Md.

H. H. Smrth, Strong, Carlisle \& Hammond Co., 1392 W. 3d Street, Cleveland, Ohio.

\section{Consumers:}

W. B. BARth, General Motors Corporation, Standards Section, 15-158 General Motors Bldg., Detroit, Mich.

Lt. Col. Harry B. Hambleton, Office of Chief of Ordnance, War Department, Washington, D. C.

A. M. Houser, Crane Company, 836 S. Michigan Ave., Chicago, Ill.

L. A. WenN, International Business Machines Co., North Street, Endicott, N. Y. H. W. Samson, Standards Department, General Electric Co., Schenectady, N. Y. Lt. Comdr. J. W. Huckert, USN, Naval Gun Factory, U. S. Navy Yard, Washington, D. C.

\section{Laboratories:}

H. W. Bearce, Interdepartmental Screw Thread Committee, National Bureau of Standards, Washington, D. C.

Earle Buckingham, Massachusetts Institute of Technology, Cambridge, Mass.

\section{HISTORY OF PROJEC'T}

In the United States the standardization of screw threads was begun with the appointment of a special committee by the Franklin Institute on April 21, 1861, for the investigation of a proper system of screw threads, bolt heads, and nuts. From this beginning there was developed a system variously known as the Franklin Institute thread, the Sellers thread, or the United States thread. Later a system having finer pitches was recommended by the Society of Automotive Engineers, and a machine-screw-thread series providing smaller sizes of screws than the United States series was recommended by the American Society of Mechanical Engineers.

On July 18, 1918, the Congress authorized the appointment of the National Screw Thread Commission, consisting of nine members, to "ascertain and establish standards for screw threads" which when "accepted and approved shall be adopted and used in the several manufacturing plants under the control of the War and Navy Departments, and, so far as practicable, in all specifications for screw threads in proposals for manufactured articles, parts, or materials to be used under the direction of these departments." The National Screw Thread Commission issued printed reports in 1921, 1924, 1928, and 1933, based upon a long series of hearings and investigations both in the United States and abroad.

While the recommendations of the NSTC are mandatory upon the War and Navy Departments, and, as far as practicable, apply also to purchases by all Government departments, it seemed desirable to determine the extent to which these standards were being applied within the industries concerned. Accordingly, on May 8, 1929, the 
National Screw Thread Commission requested the cooperation of the National Bureau of Standards to determine the extent of adoption and use of the NSTC recommendations in industry.

The hearing of the NSTC having performed all the essential functions of the general conferences normally required as a part of the procedure leading to the establishment of commercial standards, and the recommendations of the NSTC having attained national recognition and a large following, it seemed logical to proceed directly with the circulation of the essential screw-thread tables and tolerances to industry for written acceptance. This was done and resulted in the impressive roster of organizations, listed on page V of CS24-30 and CS25-30, which indicated in writing their intention of making the American National Standard Screw Threads, as set forth in CS24-30 and CS25-30, their standard of practice, effective from July 1, 1930.

First revision and consolidation.-On March 25, 1942, the Interdepartmental Screw Thread Committee, ${ }^{5}$ recognizing that the Commercial Standards CS24-30 and CS25-30 had been rendered obsolete by revisions since their publication, requested the development of revised and additional standards in line with the generally accepted commercial practice recorded in National Bureau of Standards Handbook H28.

The National Bureau of Standards established a standing committee representing manufacturers, distributors, consumers, and laboratories, which reviewed, revised, and approved for circulation within the industry the Recommended Commercial Standard for Screw Threads and Tap Drill Sizes prepared by the Bureau.

Upon written acceptance by a predominant majority of users, distributors, and producers, as listed herein, announcement was made on November 10, 1942, that the standard would become effective for new production from February 10, 1943.

5 The National Screw Thread Commission was abolished by Executive Order dated June 10, 1933. The Interdepartmental Screw Thread Committee was established September 14, 1939, by the Departments of War, Navy, and Commerce to promote uniformity in screw-thread standards in the Departments concerned. 



\section{ACCEPTANCE OF COMMERCIAL STANDARD}

If acceptance has not previously been filed, this sheet properly filled in, signed and returned will provide for the recording of your organization as an acceptor of this commercial standard.

Division of Trade Standards,

Date

National Bureau of Standards,

Washington, D. C.

Gentlemen:

Having considered the statements on the reverse side of this sheet, we accept the Commercial Standard CS24-43 as our standard of practice in the

$\begin{array}{llll}\text { Production }^{1} & \text { Distribution }^{1} & \text { Use }^{1} & \text { Testing } \\ & \end{array}$

of screw threads and tap-drill sizes.

We will assist in securing its general recognition and use, and will cooperate with the standing committee to effect revisions of the standard when necessary.

Signature of individual officer

(in ink)

(Kindly typewrite or print the following lines)

Name and title of above officer

Organization

(Fill in exactly as it should be listed)

Street address

City and State

1 Please designate which group you represent by drawing lines through the other three. Please file separate acceptances for all subsidiary companies and affiliates which should be listed separately as acceptors. In the case of related interests, trade papers, colleges, etc., desiring to record their general approval, the words "in principle" should be added after the signature. 


\section{TO THE ACCEPTOR}

The following statements answer the usual questions arising in connection with the acceptance and its significance:

1. Enforcement.-Commercial standards are commodity specifications voluntarily established by mutual consent of those concerned. They present a common basis of understanding between the producer, distributor, and consumer and should not be confused with any plan of governmental regulation or control. The United States Department of Commerce has no regulatory power in the enforcement of their provisions, but since they represent the will of the interested groups as a whole, their provisions through usage soon become established as trade customs, and are made effective through incorporation into sales contracts by means of labels, invoices and the like.

2. The acceptor's responsibility. - The purpose of commercial standards is to establish for specific commodities, nationally recognized grades or consumer criteria and the benefits therefrom will be measureable in direct proportion to their general recognition and actual use. Instances will occur when it may be necessary to deviate from the standard and the signing of an acceptance does not preclude such departures; however, such signature indicates an intention to follow the commercial standard where practicable, in the production, distribution, or consumption of the article in question.

3. The Department's responsibility.-The major function performed by the Department of Commerce in the voluntary establishment of commercial standards on a Nation-wide basis is fourfold: first, to act as an unbiased coordinator to bring all interested parties together for the mutually satisfactory adjustment of trade standards; second, to supply such assistance and advice as past experience with similar programs may suggest; third, to canvass and record the extent of acceptance and adherence to the standard on the part of producers, distributors, and users; and fourth, after acceptance, to publish and promulgate the standard for the information and guidance of buyers and sellers of the commodity.

4. Announcement and promulgation.-When the standard has been endorsed by a satisfactory majority of production or consumption in the absence of active, valid opposition, the success of the project is announced. If, however, in the opinion of the standing committee or the Department of Commerce, the support of any standard is inadequate, the right is reserved to withhold promulgation and publication. 


\section{ACCEPTORS}

The organizations and individuals listed below have accepted these dimensions as their standard of practice in the production, distribution, and use of screw threads and tap-drill sizes. Such endorsement does not signify that they may not find it necessary to deviate from the standard, nor that producers so listed guarantee all of their products in this field to conform with the requirements of this standard. Therefore specific evidence of conformity should be obtained where required.

\section{ASSOCIATIONS}

Allied Building Metal Industries, New York, N. Y.

American Association of Engineers, Chicago, III.

American Institute of Bolt, Nut, \& Rivet Manufacturers, Cleveland, Ohio.

American Railway Engineering Association, Chicago, Ill. (In Principle.)

American Supply \& Machinery Manufacturers' Association, Inc., Pittsburgh, Pa. (In Principle.)

Associated General Contractors of America, Inc., Washington, D. C.

Manufacturers Standardization Society of the Valve \& Fittings Industry, New York, N. Y.

National Association Master Plumbers, New York, N. Y.

National Retail Hardware Association, Indianapolis, Ind.

National Screw Machine Products Association, Cleveland, Ohio.

Southern Hardware Jobbers Association, Atlanta, Ga.

Southern Supply \& Machinery Distributors' Association, Inc., Atlanta, Ga.

\section{FIRIMS}

Accurate Tool Co., Detroit, Mich.

Acme Machine Tool Co., The, Cincinnati, Ohio.

Adams Co., The, Dubuque, Iowa.

Aero Supply Manufacturing Co., Inc., Corry, Pa.

Aircooled Motors Corporation, Syracuse, N. Y.

Ajax Bolt \& Screw Co., Detroit, Mich.

Allen Manufacturing Co., The, Hartford, Conn.

Almond Manufacturing Co., T. R., Ashburnham, Mass.

Aluminum \& Brass Co., Lockport; N. Y.

Aluminum Company of America, Pittsburgh, Pa.

American Bridge Co., Pittsburgh, Pa.

American Locomotive Co., Schenec$\operatorname{tady}$, N. Y.

American Manganese Bronze Co., Holmesburg, Philadelphia, Pa.

American Seating Co., Grand Rapids, Mich.

American Screw Co., Providence, R. I.

Armstrong Manufacturing Co., The, Bridgeport, Conn.

Arrow Automatic Products Corporation, New York, N. Y.

Atlantic Machine Screw Co., S. Boston, Mass.

Atlas Bolt \& Screw Co., The, Cleveland, Ohio.

Atlas Copper \& Brass Manufacturing Co., Chicago, Ill.

Autocar Co., Ardmore, Pa.

Automatic Machinery Manufacturing Corporation, Bridgeport, Conn.

Automatic Products Co., Milwaukee, Wis.

Autoscrew Co., New York, N. Y.

Avey Drilling Machine Co., The, Covington. $\mathrm{Ky}$.

Babson-Dow Manufacturing Co., Roxbury, (Boston) Mass.

Baldwin Locomotive Works, The, Philadelphia, $\mathrm{Pa}$.

Bard Manufacturing Co., Royersford, Pa.

Bath \& Co., John, Worcester, Mass.

Bausch \& Lomb Optical Co., Rochester, N. Y.

Bausch Machine Tool Co., Springfield, Mass.

Bayonne Bolt Corporation, Bayonne, N. J.

Beard Tool Co., L. O., Lancaster, Pa. 
Bell Co., Inc., The David, Buffalo, Curtis Screw Co., Inc., Buffalo, N. Y. N. Y.

Bethlehem Steel Co., Lebanon, Pa.

Bicknell Manufacturing Co., Rockland, Maine

Biglow \& Co., Inc., L. C., New York, N. Y.

Billings \& Spencer Co., The, Hartford, Conn.

Bommer Spring Hinge Co., Brooklyn, N. Y.

Boston Machine Works Co., Lynn, Mass.

Botwinik Brothers, Inc., Hamden, New Haven, Conn.

Brightman Nut \& Manufacturing Co., Sandusky, Ohio.

Brill Co., The J. G., Philadelphia, Pa.

Brown Bag Filling Machine Co., The, Fitchburg, Mass.

Brown \& Sharpe Manufacturing Co., Providence, R. I.

Brown-Wales Co., Boston, Mass.

Buckeye Traction Ditcher Co., The, Findlay, Ohio.

Buda Co., The, Harvey, Ill.

Buerk Tool Works, Buffalo, N. Y.

Buffalo Bolt Co., North Tonawanda, N. Y.

Camden Forge Co., Camden, N. J.

Cap Screw \& Nut Co. of America, Inc., New York, N. Y.

Carey Machinery \& Supply Co., Baltimore, $\mathrm{Md}$.

Central Screw Co., Chicago, 111.

Chain Belt Co., Milwaukee, Wis.

Chatillon \& Sons, John, New York, N. Y.

Chicago, Rock Island \& Pacific Railway Co., Chicago, Ill.

Chicago Screw Co., The, Chicago, Ill.

Chrysler Corporation, Detroit, Mich.

Cincinnati Planer Co., The, Cincinnati, Ohio.

City Engineering Co., The, Dayton, Ohio.

Clark, Jas., Jr., Paterson, N. J.

Clark Bros. Bolt Co., Milldale, Conn.

Clark Metal Products, Inc., Bridgeport, Conn.

Clendenin Bros. Inc., Baltimore, Md.

Cleveland Automatic Machine Co., The, Cleveland, Ohio.

Cleveland Cap Screw Co., The, Cleveland, Ohio.

Cleveland Die \& Manufacturing Co., The, Cleveland, Ohio.

Columbus Bolt Works Co., The, Columbus, Ohio.

Commonwealth Brass Corporation, Detroit, Mich.

Comtor Co., The, Waltham, Mass:

Connecticut Tool \& Engineering Co., Bridgeport, Conn.

Continental Screw Co., New Bedford, Mass.

Cox \& Sons Co., The, Bridgeton, N. J.

Crane Co., Chicago, Ill.

Dallett Co., The, Philadelphia, Pa.

Dardelet Threadlock Corporation, Detroit, Mich.

Davis \& Hemphill, Elkridge, Md.

Defiance Machine Works, Inc., Defiance, Ohio.

Detroit Nut Co., Inc., Detroit, Mich.

Detroit Plating Industries, Detroit, Mich.

Detroit Tap \& Tool Co., Detroit, Mich.

Doehler Die Casting Co., Batavia, N. Y.

Dravo Corporation Engineering Works Division, Pittsburgh, Pa.

Eastern Machine Screw Corporation, The, New Haven, Conn.

Eastman Kodak Co., Hawk-Eye Division, Rochester, N. Y.

Eastwood-Nealley Corp., Belleville, N.J.

Economy Engineering Co., The, Willoughby, Ohio.

Ekstrom, Carlson \& Co., Rockford, IIl. Electric Boat Co., Groton, Conn.

Elterich Co., Chas., New York, N. Y. (In Principle.)

Emery Industries, Inc., Cincinnati, Ohio.

Engineers Club of Philadelphia, Pa., Philadelphia, $\mathrm{Pa}$. (In Principle.)

Erie Bolt \& Nut Co., Erie, Pa.

Essley Machinery Co., The E. L., Chicago, Ill. (In Principle.)

Fairbanks, Morse \& Co., Beloit, Wis.

Federal Products Corporation, Providence, R. I.

Federal Screw Works, Detroit, Mich.

Ferry Cap \& Set Screw Co., The, Cleveland, Ohio.

Firestone Steel Prdoucts Co., Akron, Ohio.

Firestone Tire \& Rubber Co., Akron, Ohio.

Flannery Bolt Co., Bridgeville, Pa.

Fox Munitions Corporation, Philadelphia, Pa.

Foxboro Co., The, Foxboro, Mass.

General Engineering Works, Chicago, III.

General Electric Co., Schenectady, N. Y.

General Manufacturing Co., The, Waterbury, Conn.

General Motors Corporation, Detroit, Mich.

Geometric Tool Co., The, New Haven, Conn.

Gibbs \& Cox, Inc., New York, N. Y.

Gisholt Machine Co., Madison, Wis.

Globe Products Co., The, Cleveland, Ohio.

Grabler Manufacturing Co., The, Cleveland, Ohio.

Grant Manufacturing \& Machine Co., The, Bridgeport, Conn.

Graves Elevator Co., Inc., Rochester, N. Y.

Greenfield Tap \& Die Corporation, Greenfield, Mass.

Greenlee Bros. \& Co., Rockford, IIl. 
Grimm Hardware Co., Inc., W. H., Lundberg Screw Products Co., Lansing, Chicago, Ill.

Gurley, W. \& L. E., Troy, N. Y.

Haines Gauge Co., Inc., Philadelphia, $\mathrm{Pa}$.

Hardware Products Co., Inc., Boston, Mass.

Harper Co., The H. M., Chicago, Ill.

Hartford Machine Screw Co., Hartford, Conn.

Hassall, Inc., John, Brooklyn, N. Y.

Haynes Stellite Co., Kokomo, Ind.

Hodell Chain Co., The, Cleveland, Ohio.

Hood Co., R. H., Philadelphia, Pa.

Hooper Co., Inc., F. X., Glenarm, Md.

Hudson Motor Car Co., U. S. Naval Ordnance Plant, Center Line, Mich.

Illinois Iron \& Bolt Co., Carpentersville, Ill.

Imsande Screw Products Co., Cincinnati, Ohio.

Indicating Calipers Corporation, New York, N. Y.

International Business Machines Corporation, Endicott, N. Y.

International Harvester Co., Chicago, Ill.

International Machine Tool Corporation, Foster Division, Elkhart, Ind.

International-Stacey Corporation, International Derrick \& Equipment Division, Columbus, Ohio.

Isaacson Iron Works, Seattle, Wash.

Iverson \&. Laux, Inc., Chicago, Ill.

Jacobs Aircraft Engine Co., Plant No. 1, Pottstown, Pa.

Jeffrey Manufacturing Co., The, Columbus, Ohio.

Johnson Automatics Manufacturing Co., Providence, R. I.

Johnson Rule Manufacturing Co., E. P., Chicago, Ill.

Johnston \& Jennings Co., The, Cleveland, Ohio.

Jones \& Lamson Machine Co., Springfield, Vt.

Jordan Machine Products, Inc., Detroit, Mich.

Judson-Pacific Co., San Francisco, Calif.

Kaufman Manufacturing Co., L. J., Manitowoc, Wis.

King Engineering Corporation, Ann Arbor, Mich.

Kinner Motors, Inc., Glendale, Calif.

Kramer Co., C. P., Chicago, Ill.

Lamson \& Sessions Co., The, Cleveland, Ohio.

Landis Machine Co., Waynesboro, $\mathrm{Pa}$.

Lanman Co., The E. B., East Chicago, Ind.

Larson Tool \& Stamping Co., Attleboro, Mass.

Lima Locomotive Works, Inc., Lima, Ohio.

Link-Belt Ordnance Co., Chicago, Ill.

Lionel Corporation, The, Irvington, N. J.

Los Angeles Testing Laboratory, Los Angeles, Calif.

Mich.

Machined Products Co., Louisville, Ky.

MacLean-Fogg Lock Nut Co., Chicago, Ill.

Macy \& Co., Inc., R. H., New York, N. Y.

Maine Steel, Inc., South Portland, Maine.

Mann \& Co., Hutchinson, Kans.

Maryland Bolt \& Nut Co., The, Baltimore, Md.

Mattatuck Manufacturing Co., The, Waterbury, Conn.

Merrill Brothers, Maspeth, N. Y.

Mid-West Screw Products Co., St. Louis, Mo.

Milled Screw Products Co., Chicago, IIl.

Milton Manufacturing Co., The, Milton, $\mathrm{Pa}$.

Mitchell Engineering Co., The, Spring field, Ohio.

Modern Tool Works, Rochester, N. Y.

Moore, Inc., George W., Boston, Mass.

Morrow Screw \& Nut Co., Ltd., Ingersoll, Ontario, Canada.

Morse Twist Drill \& Machine Co., New Bedford, Mass.

Mueller Co., Decatur, Ill.

Murchey Machine \& Tool Co., Detroit, Mich.

Napoleon Products Co., The, Napoleon, Ohio.

National Acme Co., The, Cleveland, Ohio.

National Brass Co., Grand Rapids, Mich.

National Lock Co., Rockford, Ill.

National Machine Products Co., Detroit, Mich.

National Screw \& Manufacturing Co., The, Cleveland, Ohio.

New Britain Machine Co., The New Britain, Conn.

New York Air Brake Co., The, Watertown, N. Y.

New York Central System, New York, N. Y.

Nilson Machine Co., The A. H., Bridgeport, Conn.

North \& Judd Manufacturing Co., New Britain, Conn.

Northwest Automatic Products Corporation, Minneapolis, Minn.

Northwest Bolt \& Nut Co., Seattle, Wash.

Ohio Brass Co., The, Mansfield, Ohio.

Oliver Iron \& Steel Corporation, Pittsburgh, Pa.

Osgood Engineering Co., Boston, Mass.

Ottemiller Co., The Wm. H., York, Pa.

Pacific Car \& Foundry Co., Renton, Wash.

Packard Motor Car Co., Detroit, Mich.

Palnut Co., The, Irvington, N. J.

Parker Wire Goods Co., Worcester, Mass. 
Pawtucket Manufacturing Co., Pawtucket, R. I.

Peck, Ștow \& Wilcox Co., Southington, Conn.

Peerless Manufacturing Corporation, Louisville, $\mathrm{Ky}$.

Penn Screw \& Machine Works, Philadelphia, $\mathrm{Pa}$.

Perry Fay Co., Elyria, Ohio.

Pheoll Manufacturing Co., Chicago, Ill.

Philadelphia Hardware \& Malleable Iron Works, Inc., Philadelphia, Pa.

Pioneer Engineering \& Manufacturing Co., Detroit, Mich.

Pioneer Pump \& Manufacturing Co., Detroit, Mich.

Pittsburgh Screw \& Bolt Corporation, Pittsburgh, Pa.

Potter Tool \& Machine Works, Ine., New York, N. Y.

Pratt Manufacturing Co., William E., Joliet, Ill.

Precision Thermometer \& Instrument Co., Philadelphia, Pa.

Pullman-Standard Car Manufacturing Co., Worcester, Mass.

Quadriga Manufacturing Co., The, Chicago, IIl.

Ramsdell Manufacturing Co., Cleveland, Ohio.

Rausch Nut \& Manufacturing Co., The, Cleveland, Ohio.

Reading Hardware Corporation, Reading, Pa.

Reed Manufacturing Co., Erie, Pa.

Reed \& Prince Manufacturing Co., Worcester, Mass.

Reed Small Tool Works, Worcester, Mass.

Republic Steel Corporation, Bolt \& Nut Division of, Cleveland, Ohio.

Resistoflex Corporation, Belleville, N. J.

Reynolds Co., Hal W., Cleveland, Ohio.

Rhode Island Tool Co., Providence, R. I.

Rochester Machine Screw Co., Inc., Rochester, N. Y.

Rogers Tool Corporation, John M., Gloucester City, N. J.

Rolled Thread Die Co., Worcester, Mass.

Russell, Burdsall \& Ward Bolt \& Nut Co., Port Chester, N. Y.

Russell \& Erwin Manufacturing Co., New Britain, Conn.

Sabin Machine Co., Cleveland, Ohio.

St. Louis Screw \& Bolt Co., St. Louis, Mo.

Sargent \& Greenleaf, Inc., Rochester, N. Y.

Scherr Co., Inc., George, New York, N. Y.

Schnorr \& Co., C. H., Springdale, Pa.

Scovill Manufacturing Co., Waterbury, Conn.

Screw Machine Specialty Co., Pittsburgh, Pa.

Sears, Roebuck \& Co., Chicago, Ill.

Seymour Brass Turning Co., Seymour, Conn.
Shakeproof, Inc., Chicago, Ill.

Sheffield Corporation, The, Dayton, Ohio.

Sherman-Klove Co., The, Chicago, Ill. Simmons Machine Tool Corporation, Albany, N. Y.

Smalley-General Co., Bay City, Mich. Smith Manufacturing Co., F. H., Chicago, Ill.

Snap-On Tools Corporation, Kenosha, Wis.

South Bend Lathe Works, South Bend, Ind.

Standard Gage Co., Inc., Poughkeepsie, N. Y.

Standard Nut \& Bolt Co., Valley Falls, R. I.

Standard Shop Equipment Co., Philadelphia, $\mathrm{Pa}$.

Stevens Inc., John B., New York, N. Y. Stewart Warner Corporation, Stewart Die Casting Division, Chicago, Ill.

Strong, Carlisle \& Hammond Co., Cleveland, Ohio.

Stulz-Sickles Co., Newark, N. J.

Superior Machine \& Engineering Co., Detroit, Mich.

Superex Gage Co., Ferndale, Mich.

Taft-Peirce Manufacturing Co., The, Woonsocket, R. I.

Taylor Instrument Companies, Rochester, N. Y.

Taylor-Wharton Iron \& Steel Co., Easton, Pa.

Teer, Wickwire \& Co., Jackson, Mich. Thompson Products, Inc., Cleveland, Ohio.

Threadwell Tap \& Die Co., Greenfield, Mass.

Thwing-Albert Instrument Co., Philadelphia, Pa.

Triplex Machine Tool Co., New York, N. Y.

Triplex Screw Co., Cleveland, Ohio.

Trundle Engineering Co., The, Cleveland, Ohio.

Twining Laboratories, The, Fresno, Calif.

Union Twist Drill Co., Butterfield Division, Derby Line, Vt.

Union Twist Drill Co., S. W. Card Manufacturing Division. Mansfield, Mass.

United Drill \& Tool Corporation, Whitman \& Barnes Division, Detroit, Mich.

Upson-Walton Co., The, Cleveland, Ohio.

V \& O Press Co., Inc., The, Hudson, N. Y.

Van Keuren Co., The, Watertown, Mass.

Vard Inc., Pasadena, Calif.

Vega Aircraft Corporation, Burbank, Calif.

Virginia Polytechnic Institute, Blacksburg, Va. 
Waltham Machine Works, Waltham, |Winter Brothers Co., Wrentham, Mass. Mass.

Walworth Co., New York, N. Y.

Waterbury Button Co., Waterbury, Conn.

Waterbury Farrel Foundry \& Machine Co., Waterbury, Conn.

Weatherhead Co., The, Cleveland, Ohio.

Weiler, Edward W., New York, N. Y. (In Principle.)

Wells Tool Co., Greenfield, Mass.

Western Automatic Machine Screw Co., The, Elyria, Ohio.

Western Electric Co., Inc., New York, N. Y.

Western Machine Co., Milwaukee, Wis.

Western Union Telegraph Co., Inc., New York, N. Y.

Westinghouse Electric \& Manufacturing Co., Pittsburgh, Pa.

Williams \& Co., J. H., Buffalo, N. Y.

Woodruff \& Sons Co., The Walter W., Mt. Carmel, Conn.

Woodworkers Tool Works, Inc., Chicago, Ill.

Worthington Pump \& Machinery Corporation, Harrison, N. J.

Wright Accurate Screw Machine Products, Albert, San Francisco, Calif.

Wright Machine Co., Worcester, Mass.

\section{J. S. GOVERNMENT}

Agriculture, U. S. Department of, Washington, D. C.

Panama Canal, The, Mechanical Division, Balboa, C. Z.

Treasury Department, Washington, D. C.

Veterans Administration, Washington, D. C. 


\section{INDEX}

\section{A}

Page

American National screw threads:

Coarse-thread series

8-pitch thread series.

Extra-fine thread series.

Fine thread series.

16-pitch thread scries................ 25, 27,34, 36

12-pitch thread series........... 24, 26, 30, 32

Acceptance of standard

Acceptor's responsibility

Allowance (definition)

Angle of thread (definition)

Angle of thread (specifications)

Angle-half angle of thread (definition)

Angle-helix angle of thread (definition)

Area, mean area (definition)

Axis of screw (definition)

\section{B}

Base of thread (definition)

Basic sizc (definition)

Basic diameter:

Coarse-thread series

8-pitch-thread series

Extra-fine-thread series

Fine-thread series.

16-pitch-thread series

12-pitch-thread series

\section{C}

Classes of fits. (See Fits.)

Clearance at major diameter (specifications) ..... Clearance at minor diameter (specifications) .....

Clearance, crest (definition) . . . . . . . . . . . . . .

Clearance, terms relating to

Coarse-thread series (screw threads):

Basic diameters and thrcad data

Limiting dimensions and tolerances, classes 1 , 2, 3, and $4 \mathrm{fits}$
Tap-drill sizes. (See Tap drills.)

Tap-drill sizes. (See Tap drills.)

Committee, standing -.-_-_-_._- 53

Crest of thread (definition)

Crest clearance (definition)

\section{D}

\section{Definitions:}

\section{Allowance}

Angle of thread

(1)

Axis of a screw

Base of thread.

Basic size

Crest clearance.

Crest of thread.

Depth of engagement

Depth of thread.

External and internal threads

(1)

Finish

Fit

Half angle of thread

Helix angle of thread

Lead.

Length of engagement

Limits.

Major diameter

Minor diameter.-

Mean area.

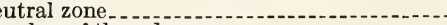

Number of threads

Pitch

Pitch diameter.

Pitch line

Definitions:-Continued. Page

Root............ 3

Screw thread.....

Side or flank

Thickness of thread.

Tolerance

Department of Commerce responsibility....... 58

Depth of engagement (definition)

Depth of thread (definition)

Depth of thread (specifications)

Diameter, major (definition)

Diameter, minor (definition)

Diameter, pitch (definition)

Diameters, basic (coarse-thread series)

Diameters, basic (fine-thread series)

Dimensions. (See Fits.)

Directions of tolerances on screw and nut (see fig. 10).

Drills. (See Tap drills.)

\section{$\mathbf{E}$}

Eight-pitch screw-thread series:

Basic diameters and thread data

Limiting dimensions and tolerances, classes 2 and 3 fits ........

Tap-drill sizes

Enforcement

Engagement, depth of

Engagement, length of

Extra-fine screw-thread series:

Basic diameters and thread data........... 39

Limiting dimensions and tolerances, classes

2 and 3 fits.

Tap-drill sizes

External thread (definition)

\section{F}

Flank or side (definition)

-

F rat at crest and root (specifications) ............

Flank - side of flank (definition)

Fine thread series:

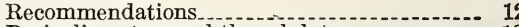

Basic diameters and thread data

Limiting dimensions and tolerances, classes 1 ,

2,3 , and 4 fits $\ldots . . . . . . . . . . . .20,22$

Tap-drill sizes ......... 45, 46

Finish (definition)

Fit (definition) Fits:

Coarse-thread series-classes 1, 2, 3, and 4 fits 8-pitch-thread series-classes 2 and 3 fits...... 28 Extra-fine-thread series-classes 2 and 3 fits 40 Fine-thread series-classes $1,2,3$ and 4 fits $--20,22$ 16-pitch-thread series-classes 2 and 3 fits... 34,36 12-pitch-thread series-classes 2 and 3 fits... 30,32

H

Half angle of thread (definition)

Helix angle of thread (definition)

History of project

\section{I}

Identification symbols

Internal thread (definition)

\section{L}

Labeling

Lead (definition)

Length of engagement (definition)

Limiting dimensions and tolerances: (See Fits.) Limits (definition)

40
8

22

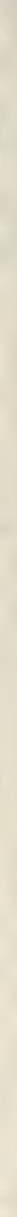

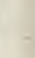


Major diameter (definition)

Mean area (definition)

Minor diameter (definition)

\section{$\mathbf{N}$}

Neutral zone (definition)

Nomenclature. (See Terms relating to classifications and tolerances.)

Notation. (See Definitions, Symbols.)

Number of threads (definition)

Numbering of tables

\section{$\mathbf{P}$}

Pitch (definition)

Pitch diameter (definition)

Pitch line (definition)

(a) Standard CS24-43. Purpose of Commercial Standard CS24-43 .....

Responsibility of Acceptor

Responsibility of Department of Commerce...

Root (definition)

$\mathbf{S}$

Screw thread (definition)

Scope of Commercial Standard C $24-43$

Side or flank (definition)

Sixteen-pitch screw-thread series:

Basic diameters and thread data

Limiting dimensions and tolerances, classes 2 and 3 fits

Tap-drill Sizes of tap drills.

Specifications:

Angle of thread

Flat at crest and root

Depth of thread.

Clearance at minor diameter

Thread series

Standing committee

Standards, Commercial list of

Symbols (identification)

\section{$\mathbf{T}$}

Tables:

Coarse-thread series:

Sizes No. $1\left(0.073^{\prime \prime}\right)$ to $4^{\prime \prime}$

Classes 1, 2, 3, and 4 fits

8-pitch-thread series

Sizes $1^{\prime \prime}$ to $6^{\prime \prime}$

Classes 2 and 3 fits
10,11

$14,16,18$ 25

Extra-fine-thread series

Sizes $1 / 4$ to $2^{\prime \prime}$

Fine-thre 2 and 3 fits

Sizes No. $0\left(0.060^{\prime \prime}\right)$ to $11 / 2^{\prime \prime}$.

Classes $1,2,3$, and 4 fits

16-pitch-thread series

Sizes $3 / 4^{\prime \prime}$ to $4^{\prime \prime}$

Classes 2 and 3 fits

12-pitch-thread series

Sizes $1 / 2^{\prime \prime}$ to $6^{\prime \prime}$

Classes 2 and 3 fits

Tap drills for No. 1 to $334^{\prime \prime}$ coarse-thread series .

Tap drills for 1 " to $312^{\prime \prime} 8$-pitch-thread series 47

Tap drills for $1 / 1^{\prime \prime}$ to $2^{\prime \prime}$ extra-fine-thread

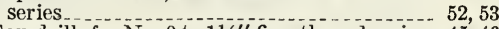

Tap drills for No. 0 to $11 / 2$ " fine-thread series. 45,46 Tap drills for $3 / 4^{\prime \prime}$ to $31 / 2^{\prime \prime}$ 16-pitch-thread

series . ...... 50,51

Tap drills for $1 / 2^{\prime \prime}$ to $31 / 2^{\prime \prime} 12$-pitch-thread series .... 48,49

Numbering of .

Scope of

Tap-drill sizes:

Coarse-thread series . . . . .

8-pitch-thread series.

Extra-fine-thread series

Fine-thread series .................. 45,46

16-pitch-thread series.-... 50,51

12-pitch-thread series

Specifications

Terms relating to classificaion and toler

ances _...

Thickness of thread (definition)

Thread, thickness of (definition)

Thread data:

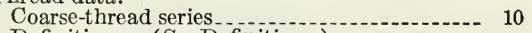

Definitions. (See Definitions.)

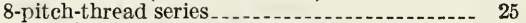

Extra-fine-thread series

Fine-thread series

16-pitch-thread series....................... 27

12-pitch-thread series ........................ 26

Specifications. (See Specifications.)

Thread series (specifications) _.............. 8

Tolerance (definition)

Tolerances (See Fits.)

Twelve-pitch screw-thread series:

Basic diameters and thread data

Limiting dimensions and tolerances, classes 2

and 3 fits.

Tap-drill sizes

\section{$\mathbf{U}$}

Uniform-pitch screw-thread series:

8-, 12-, 16-pitch-thread series. 


\section{COMMERCIAI STANDARDS}

CS No. Item

0-40. Commercial standards and their value to business (third edition).

1-42. Clinical thermometers (third edition).

2-30. Mopsticks.

$3-40$. Stoddard solvent (third edition).

4-29. Staple porcelain (all-clay) plumbing fixtures.

5-40. Pipe nipples; brass, copper, steel, and wrought iron.

6-31. Wrought-iron pipe nipples (second edition). Superseded by CS5-40.

7-29. Standard weight malleable iron or stee screwea unions.

8-41. Gage blanks (third edition).

9-33. Builders' template hardware (second edition).

10-29. Brass pipe nipples. Superseded by CS5-40.

11-41. Moisture regains of cotton yarns (second edition).

12-40. Fuel oils (fifth edition)

13-42. Dress patterns (third edition).

14-39. Boys' button-on waists, shirts, junior and polo shirts (made from woven fabrics) (second edition).

15-29. Men's pajamas.

16-29. Wall paper.

17-42. Diamond core drill fittings (third edition).

18-29. Hickory golf shafts.

19-32. Foundry patterns of wood (second edition).

$20-42$. Staple vitreous china plumbing fixtures (third edition).

21-39. Interchangeable ground-glass joints, stopcocks, and stoppers (fourth edition).

22-40. Builders' hardware (nontemplate) (second edition)

23-30. Feldspar.

24-43. Screw threads and tap-drill sizes.

25-30. Special screw threads. Superseded by CS24-43.

26-30. Aromatic red cedar closet lining.

27-36. Mirrors (second edition).

28-32. Cotton fabric tents, tarpaulins, and covers.

29-31. Staple seats for water-closet bowls.

$30-31$. Colors for sanitary ware.

31-38. Wood shingles (fourth edition).

32-31. Cotton cloth for rubber and pyroxylin coating

33-32. Knit underwear (exclusive of rayon)

34-31. Bag, case, and strap leather.

35-42. Plywcod (hardwood and eastern red cedar) (second edition).

36-33. Fourdrinier wire cloth (second edition).

37-31. Steel bone plates and screws.

38-32. Hospital rubber sheeting.

39-37. Wool and part wool blankets (second edition) (Withdrawn as commercial standard, July 14, 1941).

40-32. Surgeons' rubber gloves.

41-32. Surgeons' latex gloves.

42-35. Fiber insulating board (second edition).

43-32. Grading of sulphonated oils.

44-32. A pple wraps.

45-42. Douglas fir plywood (fifth edition).

46-40. Hosiery lengths and sizes (third edition).

47-34. Marking of gold-filled and rolled-gold-plate articles other than watchcases.

48-40. Domestic burners for Pennsylvania anthracite (underfeed type) (second edition).

49-34. Chip board, laminated chip board, and miscellaneous boards for bookbinding purposes.

50-34. Binders board for bookbinding and other purposes.

51-35. Marking articles made of silver in combination with gold.

52-35. Mohair pile fabrics (100-percent mohair plain velvet, 100 -percent mohair plain frieze, and 50 -percent mohair plain frieze).

53-35. Colors and finishes for cast stone.

54-35. Mattresses for hospitals.

55-35. Mattresses for institutions.

56-41. Oak flooring (second edition).

57-40. Book cloths, buckrams, and impregnated fabrics for bookbinding purposes except library bindings (second edition).
CS No.

Item

58-36. Woven elastic fabrics for use in overalls (overall elastic webbing).

59-41. Woven textile fabrics-testing and reporting (third edition).

60-36. Hardwood dimension lumber.

61-37. Wood-slat venetian blinds.

62-38. Colors for kitchen accessories.

63-38. Colors for bathroom accessories.

64-37. Walnut veneers.

65-43. Methods of analysis and of reporting fiber composition of textile products (second edition).

66-38. Marking of articles made wholly or in part of platinum.

67-38. Marking articles made of karat gold.

68-38. Liquid hypochlorite disinfectant, deodorant, and germicide.

69-38. Pine oil disinfectant.

70-41. Phenolic disinfectant (emulsifying type) (second edition) (published with CS71-41).

71-41. Phenolic disinfectant (soluble type) (second edition) (published with CS70-41).

72-38. Household insecticide (liquid spray type).

73-38. Old growth Douglas fir standard stock doors.

74-39. Solid hardwood wall paneling.

75-42. Automatic mechanical draft oil burners designed for domestic installations (second edition).

76-39. Hardwood interior trim and molding.

77-40. Sanitary cast-iron enameled ware.

78-40. Ground-and-polished lenses for sun glasses (second edition) (published with CS79-40).

79-40. Blown, drawn, and dropped lenses for sun glasses (second edition) (published with CS78-40).

80-41. Electric direction signal systems other than semaohore type for commercial and other vehicles subject to special motor vehicle laws (after market).

81-41. Adverse-weather lamps for vehicles (after market).

82-41. Inner-controlled spotlamps for vehicles (after market).

83-41. Clearance, marker, and identification lamps for vehicles (after market).

84-41. Electric tail lamps for vehicles (after market).

85-41. Electric license-plate lamps for vehicles (after market)

86-41. Electric stop lamps for vehicles (after market). 87-41. Red electric warning lanterns.

88-41. Liquid-burning ilares.

89-40. Hardwood stair treads and risers.

90- . (Reserved for power shovels and cranes).

91-41. Factory-fitted Douglas fir entrance doors.

92-41. Cedar, cypress, and redwood tank stock lumber.

93-41. Portable electric drills (exclusive of high frequency)

94-41. Calking lead.

95-41. Lead pipe.

96-41. Lead traps and bends.

97-42. Electric supplementary driving and passing lamps for vehicles (after market).

98-42. Artists' oil paints.

99-42. Gas floor furnaces-gravity circulating type.

100-42. Multiple-coated, porcelain-enameled steel utensils.

101-43. Flue-connected oil-burning space heaters equipped with vaporizing pot-type burners.

102- . (Reserved for Diesel and fuel-oil engines).

103-42. Cotton and rayon velour (jacquard and plain).

(E) 104-43. Warm air furnaces equipped with vaporizing pot-type oil burners.

105-43. Mineral wool; loose, granulated, or felted form, in low-temperature installations.

(E) 106-43. Boys' pajamas (made from woven fabrics).

Notice.- Those interested in commercial standards with a view toward accept-

ing them as a basis of everyday practice may secure copies of the above standards, while the supply lasts, by addressing the Division of Trade Standards, National Bureau of Standards, Washington. D. C. 